\title{
Rate-Independent Damage in Thermo-Viscoelastic Materials with Inertia
}

\author{
Giuliano Lazzaroni ${ }^{1}$ (D) Riccarda Rossi ${ }^{2}$. \\ Marita Thomas ${ }^{3}$ - Rodica Toader ${ }^{4}$
}

Received: 24 October 2014 / Revised: 22 February 2018

(C) Springer Science+Business Media, LLC, part of Springer Nature 2018

\begin{abstract}
We present a model for rate-independent, unidirectional, partial damage in viscoelastic materials with inertia and thermal effects. The damage process is modeled by means of an internal variable, governed by a rate-independent flow rule. The heat equation and the momentum balance for the displacements are coupled in a highly nonlinear way. Our assumptions on the corresponding energy functional also comprise the case of the AmbrosioTortorelli phase-field model (without passage to the brittle limit). We discuss a suitable weak formulation and prove an existence theorem obtained with the aid of a (partially) decoupled time-discrete scheme and variational convergence methods. We also carry out the asymptotic analysis for vanishing viscosity and inertia and obtain a fully rate-independent limit model for displacements and damage, which is independent of temperature.
\end{abstract}

Keywords Partial damage - Rate-independent systems · Elastodynamics · Phase-field models $\cdot$ Heat equation $\cdot$ Energetic solutions $\cdot$ Local solutions

Mathematics Subject Classification 35Q74 · 74H20 - 74R05 · 74C05 · 74F05

Giuliano Lazzaroni

giuliano.lazzaroni@unina.it

Riccarda Rossi

riccarda.rossi@unibs.it

Marita Thomas

marita.thomas@wias-berlin.de

Rodica Toader

rodica.toader@uniud.it

1 DMA, Università degli Studi di Napoli Federico II, via Cintia, Monte S. Angelo, 80126 Naples, Italy

2 DIMI, Università degli Studi di Brescia, Via Valotti 9, 25133 Brescia, Italy

3 Weierstrass Institute for Applied Analysis and Stochastics, Mohrenstr. 39, 10117 Berlin, Germany

4 DMIF, Università degli Studi di Udine, Via delle Scienze 206, 33100 Udine, Italy 


\section{Introduction}

Gradient damage models have been extensively studied in recent years, in particular in order to understand the behavior of brittle or quasi-brittle materials. In this paper we present a model for rate-independent, unidirectional, partial damage in visco-elastic materials with inertia and thermal effects. Thus we deal with a PDE system composed of the (damped) equation of elastodynamics, a rate-independent flow rule for the damage variable, and the heat equation, coupled in a highly nonlinear way. We prove an existence result basing on time-discretization and variational convergence methods, where the analytical difficulties arise from the interaction of rate-independent and rate-dependent phenomena. We study also the relationship of our model with a fully rate-independent system by time rescaling.

Following Frémond's approach [24], damage is represented through an internal variable, in the context of generalized standard materials [29]. The damage process is unidirectional, meaning that no healing is allowed; we do not use the term "irreversibility" to avoid confusion with thermodynamical notions. In our model the evolution of this variable is rate-independent: this choice is due to the consideration that, to damage a certain portion of the material, one needs a quantity of energy that is independent of the rate of damage, see e.g. [32]. Rateindependent damage has been widely explored over the last years, cf. e.g. [6,18,19,26,34, $44,67,68]$. For different studies on rate-dependent damage we refer to e.g. [8,9,22] in the isothermal case and $[3,28,62,63]$ for temperature-dependent systems.

Energy can be dissipated not only by damage growth, but also by viscosity and heat, both phenomena having a rate-dependent nature. Rate-independent processes coupled with viscosity, inertia, and also temperature have first been analyzed in the two pioneering papers [56,57], cf. also [45, Chapter 5]. Under the assumption of small strains, the momentum equation is linearized and is formulated using Kelvin-Voigt rheology and inertia. The nonlinear heat equation is coupled with the momentum balance through a thermal expansion term: this reflects the fact that temperature changes produce additional stresses. Here, we extend Roubíček's ansatz for the temperature-dependent setting to a unidirectional process, thus dealing with a discontinuous rate-independent dissipation potential, cf. (1.2) below. Existence results for an Ambrosio-Tortorelli-type system with unidirectional damage, inertia, and damping were already provided in [37] in the isothermal case.

The PDE system. More precisely, we address the analysis of the following PDE system:

$$
\rho \ddot{u}-\operatorname{div}(\mathbb{D}(z, \theta) e(\dot{u})+\mathbb{C}(z) e(u)-\theta \mathbb{B})=f_{\mathrm{V}}
$$

$$
\begin{array}{lr}
\partial \mathrm{R}_{1}(\dot{z})+\mathrm{D}_{z} G(z, \nabla z)-\operatorname{div}\left(\mathrm{D}_{\xi} G(z, \nabla z)\right)+\frac{1}{2} \mathbb{C}^{\prime}(z) e(u): e(u) \ni 0 & \text { in }(0, T) \times \Omega, \\
\dot{\theta}-\operatorname{div}(\mathbb{K}(z, \theta) \nabla \theta)=\mathrm{R}_{1}(\dot{z})+\mathbb{D}(z, \theta) e(\dot{u}): e(\dot{u})-\theta \mathbb{B}: e(\dot{u})+H & \text { in }(0, T) \times \Omega,
\end{array}
$$

where the unknowns are the displacement vector field $u$, the damage variable $z$, and the absolute temperature $\theta$, all the three being functions of the time $t \in(0, T)$ and of the position $x$ in the reference configuration of a material $\Omega$, a bounded subset of $\mathbb{R}^{d}$, with $d \in\{2,3\}$. Here, $e(u):=\frac{1}{2}\left(\nabla u+\nabla u^{\top}\right)$ denotes the linearized strain tensor.

In (1.1a), the constant $\rho>0$ is the mass density. Moreover, $\mathbb{D}(z, \theta)$ and $\mathbb{C}(z)$ are the viscous and the elastic stress tensors and are both bounded, symmetric, and positive definite on symmetric matrices, uniformly in $z$ and $\theta$. This reflects two hypotheses of the model, 
motivated by analytical reasons: first, we cannot renounce the presence of some damping in the momentum balance; second, we restrict ourselves to the case of partial damage, assuming that even in its most damaged state the material keeps some elastic properties. In order to account for the phenomenological effect that an increase of damage reduces the stored elastic energy, see e.g. [35], it is assumed that the elastic tensor $\mathbb{C}(z)$ depends monotonically on the internal variable $z$, cf. also [22,24,52].

According to the rate-independent and unidirectional nature of the damage process, $\mathrm{R}_{1}$ is a 1-homogeneous dissipation potential of the form

$$
\mathrm{R}_{1}(v):= \begin{cases}|v| & \text { if } v \leq 0 \\ +\infty & \text { otherwise }\end{cases}
$$

which enforces the internal variable $z$ to be nonincreasing in time. Indeed, we assume that $z=1$ marks the sound material and $z=0$ the most damaged state.

The gradient term $G(z, \nabla z)$ is needed to regularize damage; in particular, this term also allows for a nonconvex dependence on $z$ as in many phase-field models. Moreover, for suitable choices we retrieve the Modica-Mortola term appearing in the Ambrosio-Tortorelli functional, see Remark 2.2. The flow rule (1.1b) is given as a subdifferential inclusion, where $\partial$ denotes the subdifferential in the sense of convex analysis of $R_{1}$ while $D_{z}$ and $D_{\xi}$ stand for the Gâteaux derivatives of $G(\cdot, \xi)$ and $G(z, \cdot)$, respectively. This is a compact way to write a (semi)-stability condition of Kuhn-Tucker type.

The term $\theta \mathbb{B}$, where $\mathbb{B}$ is a fixed symmetric matrix, derives from thermodynamical considerations and is a coupling term between the momentum (1.1a) and the heat equation (1.1c). The information on the heat conductivity of the material is contained in the symmetric matrix $\mathbb{K}(z, \theta)$. We suppose that $\mathbb{K}(z, \cdot)$ satisfies subquadratic growth conditions uniformly in $z$, which are borrowed from [63] and which are in the same spirit as in [23]. These conditions are fundamental in the proof of some a priori estimates; see the discussion below (1.4) for appropriate examples from materials science.

All the aforementioned quantities are independent of time and space, whilst the external force $f_{\mathrm{V}}$ and the heat source $H$ are functions of both. The system is complemented with the natural boundary conditions

$$
\begin{aligned}
(\mathbb{D}(z, \theta) e(\dot{u})+\mathbb{C}(z) e(u)-\theta \mathbb{B}) v & =f_{\mathrm{S}} \\
u & =0 \\
\mathrm{D}_{\xi} G(z, \nabla z) v & =0 \\
\mathbb{K}(z, \theta) \nabla \theta \cdot v & =h
\end{aligned}
$$

$$
\begin{aligned}
& \text { on }(0, T) \times \partial_{\mathrm{N}} \Omega, \\
& \text { on }(0, T) \times \partial_{\mathrm{D}} \Omega, \\
& \text { on }(0, T) \times \partial \Omega, \\
& \text { on }(0, T) \times \partial \Omega,
\end{aligned}
$$

where $\partial_{\mathrm{D}} \Omega$ and $\partial_{\mathrm{N}} \Omega:=\partial \Omega \backslash \partial_{\mathrm{D}} \Omega$ are the Dirichlet and the Neumann part of the boundary, $v$ denotes the outer unit normal vector to $\partial \Omega$, and $f_{\mathrm{S}}$ and $h$ are prescribed external data depending on time and space. As for the Dirichlet data, we restrict to homogeneous boundary conditions, see Remark 2.7 for a discussion on this choice. Moreover, Cauchy conditions are given on $u(0), \dot{u}(0), z(0)$, and $\theta(0)$. We refer to Sect. 2.1 for the precise assumptions on the domain and the given data.

The energetic formulation. Due to the rate-independent character of the flow rule (1.1b) and to the nonconvexity of the underlying energy, proving the existence of solutions to the PDE system (1.1) in its pointwise form seems to be out of reach. As customary in rateindependent processes, we will resort to a weak solvability concept, based on the notion 
of energetic solution, see [40] and references therein. For fully rate-independent systems, governed (in the classical PDE-formulation) by the static momentum balance for $u$ and the rate-independent flow rule for $z$, the energetic formulation consists of two properties:

- global stability: at each time $t$ the configuration $(u(t), z(t))$ is a global minimizer of the sum of energy and dissipation;

- energy-dissipation balance: the sum of the energy at time $t$ and of the dissipated energy in $[0, t]$ equals the initial energy plus the work of external loadings.

Over the last decade, this approach has been extensively applied to several mechanical problems and in particular to fracture, see e.g. [13,14,20], and damage, see e.g. [44,67,68].

However, in a context where other rate-dependent phenomena are present, the global stability condition is too restrictive. Following $[56,57]$ we will replace it with a semistability condition, where the sum of energy and dissipation is minimized with respect to the internal variable $z$ only, while the displacement $u(t)$ is kept fixed, see also [7,59,61]. Accordingly, we will weakly formulate system (1.1) by means of

- semistability,

- the (dynamic) momentum equation in a weak sense,

- a suitable energy-dissipation balance,

- the heat equation in a weak sense.

Existence result. Theorem 2.6 states the existence of energetic solutions to the initialboundary value problem for system (1.1). For the proof we rely on a well-established method for showing existence for rate-independent processes [40], adjusted to the coupling with viscosity, inertia, and temperature in [57]. Although we follow the approach of the latter paper, let us point out that the results therein do not account for some properties of our model, namely,

- the unidirectionality of damage, see (1.2),

- the dependence of the viscous tensor $\mathbb{D}(z, \theta)$ on damage and temperature.

These features are important for the modeling of volume-damage, as well as for the phasefield approximation of fracture and surface damage models, see also Remark 2.2, and cause some analytical difficulties.

As in many works on rate-independent systems, our existence proof is based on timediscretization and approximation by means of solutions to incremental problems. Differently from [57], in our discrete scheme the approximate flow rule is decoupled from the other two equations, which may produce more efficient numerical simulations. Moreover, the assumption of a constant heat capacity allows us to avoid a so-called enthalpy transformation and, together with the subquadratic growth of the heat conductivity, to deduce a priori estimates and the positivity of the temperature by carefully adapting the methods developed in $[23,63]$.

When taking the time discrete-to-continuous limit, we first pass to the limit in the weak momentum balance. From this we also deduce a (time-continuous) mechanical energy inequality by lower semicontinuity arguments. Next we pass to the limit in the semistability inequality using so-called mutual recovery sequences. As a further step we verify that the mechanical energy balance is satisfied as an equality: this follows from the momentum balance and the semistability so far obtained. This result allows us to conclude the convergence of the viscous dissipation terms, which, in turn, is crucial for the limit passage in the heat equation. See Sects. 4.1-4.3. 
Some remarks on the thermal properties of system (1.1) and its applicability. For the thermodynamical derivation of the PDE system (1.1) one may follow the thermomechanical modeling by Frémond in [24, Chapter 12] or Roubíček in [57]. In particular, the free energy density associated with (1.1) is given by

$$
F(e(u), z, \nabla z, \theta):=\frac{1}{2} \mathbb{C}(z) e(u): e(u)+G(z, \nabla z)+\varphi(\theta)-\theta \mathbb{B}: e(u),
$$

which leads to the entropy density $S$ and the internal energy density $U$ of the form

$$
\begin{aligned}
S(e(u), z, \nabla z, \theta) & =-\partial_{\theta} F=\mathbb{B}: e(u)-\varphi^{\prime}(\theta), \\
U(e(u), z, \nabla z, \theta) & =F+\theta S=\frac{1}{2} \mathbb{C}(z) e(u): e(u)+G(z, \nabla z)+\varphi(\theta)-\theta \varphi^{\prime}(\theta),
\end{aligned}
$$

where $\varphi$ is a function such that $c_{\mathrm{V}}(\theta):=\partial_{\theta} U=-\theta \varphi^{\prime \prime}(\theta)$ is the specific heat capacity, and $S$ and $U$ satisfy a Gibbs' relation: $\partial_{\theta} U=\theta \partial_{\theta} S$. Starting from the entropy equation, which balances the changes of entropy with the heat flux and the heat sources given by the dissipation rate and the external sources $H$,

$$
\theta \partial_{\theta} S \dot{\theta}+\operatorname{div} j=\mathrm{R}_{1}(\dot{z})+(\mathbb{D}(z, \theta) e(\dot{u})-\theta \mathbb{B}): e(\dot{u})+H,
$$

and then invoking Fourier's law $j=-\mathbb{K}(z, \theta) \nabla \theta$ as well as the above Gibbs' relation, the choice $\varphi(\theta)=\theta(1-\log \theta)$ indeed results in the heat equation (1.1c) with $c_{\mathrm{V}}(\theta)=$ const. $=1$.

In fact, the temperature dependence of the heat capacity can be described by the classical Debye model, see e.g. [69, Sect. 4.2, p. 761]. In a first approximation it predicts a cubic growth of $c_{\mathrm{V}}$ with respect to temperature up to a certain, material-specific temperature, the so-called Debye temperature $\theta_{\mathrm{D}}$, whereas for $\theta \gg \theta_{\mathrm{D}}$ it can be approximated by $c_{\mathrm{V}} \equiv$ const. Thus, the use of (1.1c) with $c_{\mathrm{V}}(\theta)=$ const. (normalized to $c_{\mathrm{V}}(\theta)=1$ for shorter presentation) is justified if the temperature range of application is assumed to be above Debye temperature, i.e., $\theta \gg \theta_{\mathrm{D}}$. Indeed, our main existence Theorem 2.6, see also Proposition 3.2, contains an enhanced positivity estimate, which ensures that the temperature $\theta$, as a component of an energetic solution $(u, z, \theta)$, always stays above a tunable threshold (to be tuned to $\theta_{\mathrm{D}}$ ), provided that the initial temperature and the heat sources $H$ are suitably large, see (2.16).

In this context, let us here also allude to our hypothesis on the heat conductivity tensor $\mathbb{K}(z, \theta)$, which is assumed to have subquadratic growth in $\theta$, see (2.6b). According to experimental findings, cf. [16,31], polymers such as e.g. polymethylmethacrylate (PMMA), exhibit such a subquadratic growth of the heat conductivity. In contrast, for metals the heat conductivity is ruled by the electron thermal conductivity. For this, the Wiedemann-Franz law states a linear dependence on the temperature, cf. [11, Chapter 17]. Moreover, let us mention that the analytical results in [23] are obtained under the assumption of superquadratic growth, which is justified by the examples on nonlinear heat conduction given in [70], that are related to radiation heat conduction or electron/ion heat conduction in a plasma. Thus, in conclusion, the thermal properties of our model rather comply with polymers than with metals.

Vanishing viscosity and inertia. Finally, in Sect. 5 ahead we will address the analysis of system (1.1) as the rates of the external load and of the heat sources become slower and slower. Therefore, we will rescale time by a factor $\varepsilon$ and perform the asymptotic analysis as $\varepsilon \downarrow 0$ of the rescaled system, i.e. with vanishing viscosity and inertia in the momentum equation, and vanishing viscosity in the heat equation. Before entering into the details of our result, let us briefly overview some related literature.

On the one hand, the asymptotic analysis for vanishing viscosity and inertia of the sole momentum balance has been the subject of earlier work: we refer, e.g., to [50] for study of the 
purely elastic limit of dynamic viscoelastic solutions to a frictional contact problem, in terms of a graph solution notion. This problem was approached from a more abstract viewpoint in [46], with applications to finite-dimensional mechanical systems featuring elastic-plastic behavior with linear hardening in [42]. On the other hand, a well-established approach to fully rate-independent systems consists in viscously regularizing the rate-independent flow rule for the internal variable (typically coupled with a purely elastic equilibrium equation for the displacements), and taking the vanishing-viscosity limit. This leads to parameterized/BV solutions, encoding information on the energetic behavior of the system at jumps, see e.g. [12, $17,48,49]$, as well as e.g. [33,34,39] for applications to fracture and damage. We also mention $[1,53]$ for finite-dimensional singularly perturbed second order potential-type equations. The convergence of kinetic variational inequalities to rate-independent quasistatic variational inequalities was tackled in [43].

Let us point out that our analysis is substantially different from the "standard" vanishingviscosity approach to rate-independent systems, since in our context viscosity (and inertia for the momentum equation) vanish in the heat and momentum balances, only, while we keep the flow rule for the damage parameter rate-independent. In fact, our study is akin to the vanishing-viscosity and inertia analysis that has been addressed, in the momentum equation only, for isothermal, rate-independent processes with dynamics in [56,58], leading to an energetic-type notion of solution. We also refer to $[15,66]$ for a combined vanishingviscosity limit in the momentum equation and in the flow rule, in the cases of perfect plasticity and delamination, respectively.

The coupling with the temperature equation attaches an additional difficulty to our own vanishing-viscosity analysis. Because of this, it will be essential to assume an appropriate scaling of the tensor of heat conduction coefficients: in fact, we shall require that the conductivity matrix ( $\mathbb{K}$ in (1.1c)) diverges as inertia and viscosity vanish. This reflects the fact that in the slow-loading regime heat propagates at infinite speed. Thus, in the slow-loading limit we will obtain that the temperature is spatially constant and its evolution is fully decoupled from the one of the mechanical variables. Indeed, in Theorem 5.3. we will prove convergence as $\varepsilon \downarrow 0$ of energetic solutions $\left(u_{\varepsilon}, z_{\varepsilon}, \theta_{\varepsilon}\right)$ of the rescaled system to a triple $(u, z, \Theta)$ such that

- $(u, z)$ is local solution (according to the notion introduced in $[41,58]$ ) to the (fully rate-independent) system consisting of the static momentum balance and of the rateindependent flow rule for damage;

- under a suitable scaling condition on the heat sources, the spatially constant function $\Theta$ satisfies an ODE that involves a nonnegative defect measure arising from the limit of the viscoelastic dissipation term.

Plan of the paper. The assumptions on the material quantities and the statement of the existence results for energetic solutions are given in Sect. 2. In Sect. 3 we present the properties of time-discrete solutions, hence in Sect. 4 we prove the main theorem by passing to the time-continuous limit by variational convergence techniques. Finally, Sect. 5 is devoted to the asymptotics for vanishing viscosity and inertia.

\section{Setup and Main Result}

Notation: Throughout this paper, for a given Banach space $X$ we will denote by $\langle\cdot, \cdot\rangle_{X}$ the duality pairing between $X^{*}$ and $X$, and by $\mathrm{BV}([0, T] ; X)$, resp. $\mathrm{C}_{\text {weak }}^{0}([0, T] ; X)$, the space of the bounded variation, resp. weakly continuous, functions with values in $X$. Notice that we shall consider any $v \in \mathrm{BV}([0, T] ; X)$ to be defined at all $t \in[0, T]$. We also mention that 
the symbols $c, C, C^{\prime} \ldots$ will be used to denote a positive constant depending on given data, and possibly varying from line to line. Furthermore in proofs, the symbols $I_{i}, i=1, \ldots$, will be place-holders for several integral terms popping up in the various estimates. We warn the reader that we will not be self-consistent with the numbering so that, for instance, the symbol $I_{1}$ will occur in several proofs with different meanings.

\subsection{Assumptions}

We now specify the assumptions on the domain $\Omega$, on the nonlinear functions featured in (1.1), on the initial data, and on the loading and source terms, under which our existence result, Theorem 2.6, holds. Let us mention in advance that, in order to simplify the exposition in Sects. 2-4, and in view of the analysis for vanishing viscosity and inertia in Sect. 5, cf. (5.32), we will suppose that the matrix of thermal expansion coefficients is a given symmetric matrix $\mathbb{B} \in \mathbb{R}_{\text {sym }}^{d \times d}$. We instead allow the elasticity and viscosity tensors to depend on the state variables $z$ and $(z, \theta)$, respectively, thus we need to impose suitable growth and coercivity conditions. We will also make growth assumptions for the matrix of heat conduction coefficients, which are suited for our analysis and which are in the line of $[23,63]$. These growth conditions will play a key role in the derivation of estimates for the temperature $\theta$, in that it will allow us to cope with the quadratic right-hand side of (1.1c). Before detailing the standing assumptions of this paper, let us mention that, to ease the presentation, we will assume the functions of the temperature featuring in the model to be defined also for nonpositive values of $\theta$. At any rate, later on we will prove the existence of solutions such that the temperature is bounded from below by a positive constant, see (2.14)-(2.16).

Assumptions on the domain. We assume that

$\Omega \subset \mathbb{R}^{d}, d \in\{2,3\}$, is a bounded domain with Lipschitz-boundary $\partial \Omega$ such that $\partial_{\mathrm{D}} \Omega \subset \partial \Omega$ is nonempty and relatively open and $\partial_{\mathrm{N}} \Omega:=\partial \Omega \backslash \partial_{\mathrm{D}} \Omega$.

Moreover, we will use the following notation for the state spaces for $u$ and $z$ :

$$
\begin{aligned}
H_{\mathrm{D}}^{1}\left(\Omega ; \mathbb{R}^{d}\right) & :=\left\{v \in H^{1}\left(\Omega ; \mathbb{R}^{d}\right): v=0 \text { on } \partial_{\mathrm{D}} \Omega \text { in the trace sense }\right\}, \\
z & :=\left\{z \in W^{1, q}(\Omega): z \in[0,1] \text { a.e. in } \Omega\right\}
\end{aligned}
$$

with fixed $q>1$, cf. (2.5d). Analogous notation will be employed for the Sobolev spaces $W_{\mathrm{D}}^{1, \gamma}, \gamma \geq 1$.

Assumptions on the material tensors. We require that the tensors $\mathbb{B} \in \mathbb{R}^{d \times d}, \mathbb{C}: \mathbb{R} \rightarrow$ $\mathbb{R}^{d \times d \times d \times d}$, and $\mathbb{D}: \mathbb{R} \times \mathbb{R} \rightarrow \mathbb{R}^{d \times d \times d \times d}$ fulfill

$\mathbb{B} \in \mathbb{R}_{\text {sym }}^{d \times d}$ and set $C_{\mathbb{B}}:=|\mathbb{B}|$,

$\mathbb{C} \in \mathrm{C}^{0,1}\left(\mathbb{R} ; \mathbb{R}^{d \times d \times d \times d}\right)$ and $\mathbb{D} \in \mathrm{C}^{0}\left(\mathbb{R} \times \mathbb{R} ; \mathbb{R}^{d \times d \times d \times d}\right)$,

$\mathbb{C}(z), \mathbb{D}(z, \theta) \in \mathbb{R}_{\mathrm{sym}}^{d \times d \times d \times d}$ and are positive definite for all $z \in \mathbb{R}, \theta \in \mathbb{R}$,

$\exists C_{\mathbb{C}}^{1}, C_{\mathbb{C}}^{2}>0 \forall z \in \mathbb{R} \forall A \in \mathbb{R}_{\mathrm{sym}}^{d \times d}: \quad C_{\mathbb{C}}^{1}|A|^{2} \leq \mathbb{C}(z) A: A \leq C_{\mathbb{C}}^{2}|A|^{2}$,

$\exists C_{\mathbb{D}}^{1}, C_{\mathbb{D}}^{2}>0 \forall z \in \mathbb{R} \forall \theta \in \mathbb{R} \forall A \in \mathbb{R}_{\mathrm{sym}}^{d \times d}: C_{\mathbb{D}}^{1}|A|^{2} \leq \mathbb{D}(z, \theta) A: A \leq C_{\mathbb{D}}^{2}|A|^{2}$. 
In the expressions above, $\mathbb{R}_{\text {sym }}^{d \times d}$ denotes the subset of symmetric matrices in $\mathbb{R}^{d \times d}$ and $\mathbb{R}_{\mathrm{sym}}^{d \times d \times d \times d}$ is the subset of symmetric tensors in $\mathbb{R}^{d \times d \times d \times d}$. In particular,

$\mathbb{C}(z)_{i j k l}=\mathbb{C}(z)_{j i k l}=\mathbb{C}(z)_{i j l k}=\mathbb{C}(z)_{k l i j}$ and $\mathbb{D}(z, \theta)_{i j k l}=\mathbb{D}(z, \theta)_{j i k l}=\mathbb{D}(z, \theta)_{i j l k}=\mathbb{D}(z, \theta)_{k l i j}$.

In addition to (2.3), we impose that $\mathbb{C}(\cdot)$ is monotonically nondecreasing, i.e.,

$$
\forall A \in \mathbb{R}_{\mathrm{sym}}^{d \times d} \forall 0 \leq z_{1} \leq z_{2} \leq 1: \quad \mathbb{C}\left(z_{1}\right) A: A \leq \mathbb{C}\left(z_{2}\right) A: A .
$$

Assumptions on the damage regularization. We require that $G: \mathbb{R} \times \mathbb{R}^{d} \rightarrow \mathbb{R} \cup\{\infty\}$ fulfills

$$
\text { Indicator: For every }(z, \xi) \in \mathbb{R} \times \mathbb{R}^{d}: \quad G(z, \xi)<\infty \Rightarrow z \in[0,1]
$$

Continuity: $G$ is continuous on its $\operatorname{domain} \operatorname{dom}(G), \quad G \geq 0, \quad$ and $\quad G(0,0)=0$;

Convexity: For every $z \in \mathbb{R}, G(z, \cdot)$ is convex;

Growth: There exist constants $q>1$ and $C_{G}^{1}, C_{G}^{2}>0$ such that for every $(z, \xi) \in \operatorname{dom}(G)$

$$
C_{G}^{1}\left(|\xi|^{q}-1\right) \leq G(z, \xi) \leq C_{G}^{2}\left(|\xi|^{q}+1\right) .
$$

Remark 2.1 (Properties of the regularizing term) Since we are encompassing the feature that $z(\cdot, x)$ is decreasing for almost all $x \in \Omega$, starting from an initial datum $z_{0} \in[0,1]$ a.e. in $\Omega$, the $z$-component of any energetic solution to (1.1) will fulfill $z(t, x) \leq 1$ a.e. in $\Omega$. Therefore, we could weaken (2.5a) and just require that the domain of $G$ is a subset of $[0, \infty)$.

Furthermore, we may require the third of (2.5b) without loss of generality, since adding a constant to $G$ shall not affect our analysis.

Further observe that the above assumptions (2.5) ensure that the integral functional

$$
\mathcal{G}: L^{r}(\Omega) \times L^{q}\left(\Omega ; \mathbb{R}^{d}\right) \rightarrow \mathbb{R} \cup\{\infty\}, \quad \mathcal{G}(z, \xi):=\int_{\Omega} G(z, \xi) \mathrm{d} x
$$

is lower semicontinuous with respect to strong convergence in $L^{r}(\Omega)$ for any $r \in[1, \infty)$ and weak convergence in $L^{q}\left(\Omega ; \mathbb{R}^{d}\right)$, cf. e.g. [21, Theorem 7.5, p. 492]. In addition, $\mathcal{G}$ is continuous with respect to strong convergence in $\left(L^{r}(\Omega) \times L^{q}\left(\Omega ; \mathbb{R}^{d}\right)\right) \cap \operatorname{dom}(G)$.

Remark 2.2 (Example: Phase-field approximation of fracture) Starting from the work of Ambrosio and Tortorelli [2], gradient damage models have been extensively used in recent years to predict crack propagation in brittle or quasi-brittle materials, by means of phasefield approximation [4]. In this approach, a sharp crack is regularized by defining an internal variable that interpolates continuously between sound and fractured material. In the mathematical literature, evolutionary problems for phase-field models were considered for instance in the fully quasistatic case [25], in viscoelasticity as a gradient flow [5], and in dynamics [37], always for isothermal systems. A thermodynamical model for regularized fracture with inertia was proposed and treated numerically e.g. in [52]. The passage to the limit from phasefield to sharp crack, though successfully treated in the quasistatic [25] and in the viscous case [5], is by now an open problem in dynamics and is outside the scope of this contribution.

In this context, typical examples for the regularizing term are functionals of ModicaMortola type,

$$
\mathcal{G}_{\mathrm{MM}}^{q}(z, \nabla z)=\int_{\Omega} G_{\mathrm{MM}}^{q}(z, \nabla z) \mathrm{d} x \quad \text { with } G_{\mathrm{MM}}^{q}(z, \nabla z):=|\nabla z|^{q}+W(z)+I_{[0,1]}(z),
$$

\section{照 Springer}


where $q>1, W$ is a suitable potential, and $I_{[0,1]}(z):=0$ if $z \in[0,1], I_{[0,1]}(z):=+\infty$ otherwise. Such regularization agrees with the above assumptions up to an additive constant.

Notice that in Sect. 3, to construct discrete solutions, we will consider unilateral minimum problems of the type

$$
\min _{z \in \mathcal{Z}}\left\{\int_{\Omega} \frac{1}{2} \mathbb{C}(z) e(u): e(u) \mathrm{d} x+\int_{\Omega} G(z, \nabla z) \mathrm{d} x+\mathcal{R}_{1}(z-\bar{z})\right\}
$$

for given $u \in H_{\mathrm{D}}^{1}\left(\Omega ; \mathbb{R}^{d}\right)$ and a given $\bar{z} \in Z$ defined in (2.2). Setting $\mathbb{C}(z):=\left(z^{2}+\delta\right) I$ with $\delta>0$, and $G:=G_{\mathrm{MM}}^{2}$ with $W(z):=\frac{1}{2}\left(1+z^{2}\right)$, the minimum problem is equivalent to

$$
\min _{0 \leq z \leq \bar{z}}\left\{\int_{\Omega}\left(\frac{1}{2}\left(z^{2}+\delta\right)|e(u)|^{2} \mathrm{~d} x+\int_{\Omega} \frac{1}{2 \delta}(1-z)^{2} \mathrm{~d} x+\int_{\Omega} \delta|\nabla z|^{2} \mathrm{~d} x\right\},\right.
$$

that is the classical minimization of the Ambrosio-Tortorelli functional, see [2,25]. The generalization to $G=G_{\mathrm{MM}}^{q}$ with $q>1$ was considered in [30]. In this case one may want an effective dependence of the viscous tensor on $z$, choosing $\mathbb{D}(z, \theta)=\mathbb{C}(z)$ as in [37].

Assumptions on the heat conductivity. On $\mathbb{K}: \mathbb{R} \times \mathbb{R} \rightarrow \mathbb{R}^{d \times d}$ we assume that

$$
\begin{aligned}
& \mathbb{K} \in \mathrm{C}^{0}\left(\mathbb{R} \times \mathbb{R} ; \mathbb{R}^{d \times d}\right), \quad \mathbb{K}(z, \theta) \in \mathbb{R}_{\mathrm{sym}}^{d \times d} \text { for all } z \in \mathbb{R}, \theta \in \mathbb{R}, \\
& \exists \kappa \in\left(1, \kappa_{d}\right) \exists c_{1}, c_{2}>0 \forall(z, \theta) \in \mathbb{R} \times \mathbb{R} \quad \forall \xi \in \mathbb{R}^{d}:\left\{\begin{array}{l}
c_{1}\left(|\theta|^{\kappa}+1\right)|\xi|^{2} \leq \mathbb{K}(z, \theta) \xi \cdot \xi, \\
|\mathbb{K}(z, \theta)| \leq c_{2}\left(|\theta|^{\kappa}+1\right),
\end{array}\right.
\end{aligned}
$$

where $\kappa_{d}=5 / 3$ for $d=3$ and $\kappa_{d}=2$ for $d=2$.

The bound $\kappa_{d}$ essentially comes into play in the derivation of the Fifth a priori estimate (cf. the proof of Proposition 3.4), and when passing from time-discrete to continuous in the heat equation, cf. Proposition 4.9. Essentially, it arises as a consequence of the enhanced integrability of the approximating temperature variables obtained by interpolation in (3.32k).

Assumptions on the initial data. We impose that

$$
u_{0} \in H_{\mathrm{D}}^{1}\left(\Omega ; \mathbb{R}^{d}\right), \quad \dot{u}_{0} \in L^{2}\left(\Omega ; \mathbb{R}^{d}\right), \quad z_{0} \in \mathcal{Z},
$$

$$
\theta_{0} \in L^{1}(\Omega), \quad \text { and } \theta_{0} \geq \theta_{*}>0 \text { a.e. in } \Omega,
$$

where the state spaces $H_{\mathrm{D}}^{1}\left(\Omega ; \mathbb{R}^{d}\right)$ and $Z$ are defined in (2.2).

Assumptions on the loading and source terms. On the data $f_{\mathrm{V}}, f_{\mathrm{S}}, H$, and $h$ we require that

$$
\begin{aligned}
& f_{\mathrm{V}} \in H^{1}\left(0, T ; H_{\mathrm{D}}^{1}\left(\Omega ; \mathbb{R}^{d}\right)^{*}\right), \quad f_{\mathrm{S}} \in H^{1}\left(0, T ; L^{2}\left(\partial_{\mathrm{N}} \Omega ; \mathbb{R}^{d}\right)\right), \\
& H \in L^{1}\left(0, T ; L^{1}(\Omega)\right) \cap L^{2}\left(0, T ; H^{1}(\Omega)^{*}\right), \quad H \geq 0 \text { a.e. in }(0, T) \times \Omega, \\
& h \in L^{1}\left(0, T ; L^{2}(\partial \Omega)\right), \quad h \geq 0 \text { a.e. in }(0, T) \times \partial \Omega .
\end{aligned}
$$

For later convenience, we also introduce $f:[0, T] \rightarrow H_{\mathrm{D}}^{1}\left(\Omega ; \mathbb{R}^{d}\right)^{*}$ defined by

$$
\langle f(t), v\rangle_{H_{\mathrm{D}}^{1}\left(\Omega ; \mathbb{R}^{d}\right)}:=\left\langle f_{\mathrm{V}}(t), v\right\rangle_{H_{\mathrm{D}}^{1}\left(\Omega ; \mathbb{R}^{d}\right)}+\int_{\partial_{\mathrm{N}} \Omega} f_{\mathrm{S}} \cdot v \mathrm{~d} \mathcal{H}^{d-1}(x) \quad \text { for all } v \in H_{\mathrm{D}}^{1}\left(\Omega ; \mathbb{R}^{d}\right) .
$$

It follows from (2.8a) that $f \in H^{1}\left(0, T ; H_{\mathrm{D}}^{1}\left(\Omega ; \mathbb{R}^{d}\right)^{*}\right)$. 


\subsection{Weak Formulation and Main Existence Result}

As already mentioned, following [57], the energetic formulation of (the initial-boundary value problem associated with) system (1.1) consists of the variational formulation of the momentum and of the heat equations (1.1a) and (1.1c), with suitable test functions, and of a semistability condition joint with a mechanical energy balance, providing the weak formulation of the damage equation (1.1b). The latter relations feature the mechanical (quasistatic) energy associated with (1.1), i.e.,

$$
\mathcal{E}(t, u, z):=\int_{\Omega}\left(\frac{1}{2} \mathbb{C}(z) e(u): e(u)+G(z, \nabla z)\right) \mathrm{d} x-\langle f(t), u\rangle_{H_{\mathrm{D}}^{1}\left(\Omega ; \mathbb{R}^{d}\right)},
$$

as well as the rate-independent dissipation potential, given as the integrated version of (1.2)

$$
\mathcal{R}_{1}(\dot{z}):=\int_{\Omega} \mathrm{R}_{1}(\dot{z}) \mathrm{d} x .
$$

In Definition 2.3 below, the choice of the test functions for the weak momentum equation reflects the regularity (2.11a) required for $u$, which in turn will derive from the standard energy estimates that can be performed on system (1.1). As we will see, such estimates only yield $\theta \in L^{\infty}\left(0, T ; L^{1}(\Omega)\right)$. In fact, the further regularity (2.11c) for $\theta$ shall result from a careful choice of test functions for the time-discrete version of $(1.1 \mathrm{c})$, and from refined interpolation arguments, drawn from [23]. Finally, the BV $\left([0, T] ; W^{2, d+\delta}(\Omega)^{*}\right)$-regularity for $\theta$ follows from a comparison argument. The choice of the test functions in (2.12d) is the natural one in view of (2.11).

Definition 2.3 (Energetic solution (2.11)-(2.12)) Given a quadruple of initial data $\left(u_{0}, \dot{u}_{0}\right.$, $\left.z_{0}, \theta_{0}\right)$ satisfying (2.7), we call a triple $(u, z, \theta)$ an energetic solution of the Cauchy problem for the PDE system (1.1) complemented with the boundary conditions (1.3) if

$$
\begin{aligned}
& u \in H^{1}\left(0, T ; H_{\mathrm{D}}^{1}\left(\Omega ; \mathbb{R}^{d}\right)\right) \cap W^{1, \infty}\left(0, T ; L^{2}\left(\Omega ; \mathbb{R}^{d}\right)\right), \\
& z \in L^{\infty}\left(0, T ; W^{1, q}(\Omega)\right) \cap L^{\infty}((0, T) \times \Omega) \cap \mathrm{BV}\left([0, T] ; L^{1}(\Omega)\right), \\
& z(t, x) \in[0,1] \text { for a.a. }(t, x) \in(0, T) \times \Omega, \\
& \theta \in L^{2}\left(0, T ; H^{1}(\Omega)\right) \cap L^{\infty}\left(0, T ; L^{1}(\Omega)\right) \cap \mathrm{BV}\left([0, T] ; W^{2, d+\delta}(\Omega)^{*}\right),
\end{aligned}
$$

such that the triple $(u, z, \theta)$ complies with the initial conditions

$$
u(0)=u_{0}, \quad \dot{u}(0)=\dot{u}_{0}, \quad z(0)=z_{0}, \quad \theta(0)=\theta_{0} \quad \text { a.e. in } \Omega,
$$

and with the following properties:

- unidirectionality: for a.a. $x \in \Omega$, the function $z(\cdot, x):[0, T] \rightarrow[0,1]$ is nonincreasing; - semistability: for every $t \in[0, T]$

$$
\forall \tilde{z} \in \mathcal{Z}: \quad \mathcal{E}(t, u(t), z(t)) \leq \mathcal{E}(t, u(t), \tilde{z})+\mathcal{R}_{1}(\tilde{z}-z(t)),
$$

where $Z$ is defined in (2.2);

- weak formulation of the momentum equation: for all $t \in[0, T]$

$$
\begin{aligned}
& \rho \int_{\Omega} \dot{u}(t) \cdot v(t) \mathrm{d} x-\rho \int_{0}^{t} \int_{\Omega} \dot{u} \cdot \dot{v} \mathrm{~d} x \mathrm{~d} s \\
& \quad+\int_{0}^{t} \int_{\Omega}(\mathbb{D}(z, \theta) e(\dot{u})+\mathbb{C}(z) e(u)-\theta \mathbb{B}): e(v) \mathrm{d} x \mathrm{~d} s \\
& =\rho \int_{\Omega} \dot{u}_{0} \cdot v(0) \mathrm{d} x+\int_{0}^{t}\langle f, v\rangle_{H_{\mathrm{D}}^{1}\left(\Omega ; \mathbb{R}^{d}\right)} \mathrm{d} s
\end{aligned}
$$

\section{照 Springer}


for all test functions $v \in L^{2}\left(0, T ; H_{\mathrm{D}}^{1}\left(\Omega ; \mathbb{R}^{d}\right)\right) \cap W^{1,1}\left(0, T ; L^{2}\left(\Omega ; \mathbb{R}^{d}\right)\right)$;

- mechanical energy equality: for all $t \in[0, T]$

$$
\begin{aligned}
& \frac{\rho}{2} \int_{\Omega}|\dot{u}(t)|^{2} \mathrm{~d} x+\mathcal{E}(t, u(t), z(t))+\int_{\Omega}\left(z_{0}-z(t)\right) \mathrm{d} x \\
& \quad+\int_{0}^{t} \int_{\Omega}(\mathbb{D}(z, \theta) e(\dot{u})-\theta \mathbb{B}): e(\dot{u}) \mathrm{d} x \mathrm{~d} s \\
& \quad=\frac{\rho}{2} \int_{\Omega}\left|\dot{u}_{0}\right|^{2} \mathrm{~d} x+\mathcal{E}\left(0, u_{0}, z_{0}\right)+\int_{0}^{t} \partial_{t} \mathcal{E}(s, u(s), z(s)) \mathrm{d} s,
\end{aligned}
$$

where $\partial_{t} \mathcal{E}(t, u, z)=-\langle\dot{f}(t), u\rangle_{H_{\mathrm{D}}^{1}\left(\Omega ; \mathbb{R}^{d}\right)}$;

- weak formulation of the heat equation: for all $t \in[0, T]$

$$
\begin{aligned}
& \langle\theta(t), \eta(t)\rangle_{W^{2, d+\delta}(\Omega)}-\int_{0}^{t} \int_{\Omega} \theta \dot{\eta} \mathrm{d} x \mathrm{~d} s+\int_{0}^{t} \int_{\Omega} \mathbb{K}(\theta, z) \nabla \theta \cdot \nabla \eta \mathrm{d} x \mathrm{~d} s \\
& =\int_{\Omega} \theta_{0} \eta(0) \mathrm{d} x+\int_{0}^{t} \int_{\Omega} \eta|\dot{z}| \mathrm{d} x \mathrm{~d} s+\int_{0}^{t} \int_{\Omega}(\mathbb{D}(z, \theta) e(\dot{u}): e(\dot{u})-\theta \mathbb{B}): e(\dot{u}) \eta \mathrm{d} x \mathrm{~d} s \\
& \quad+\int_{0}^{t} \int_{\partial \Omega} h \eta \mathrm{d} \mathcal{H}^{d-1}(x) \mathrm{d} s+\int_{0}^{t} \int_{\Omega} H \eta \mathrm{d} x \mathrm{~d} s
\end{aligned}
$$

for all test functions $\eta \in H^{1}\left(0, T ; L^{2}(\Omega)\right) \cap \mathrm{C}^{0}\left([0, T] ; W^{2, d+\delta}(\Omega)\right)$, for some fixed $\delta>0$. Here and in what follows, $|\dot{z}|$ denotes the total variation measure of $z$ (i.e., the heat produced by the rate-independent dissipation), which is defined on every closed set of the form $\left[t_{1}, t_{2}\right] \times C \subset[0, T] \times \bar{\Omega}$ by

$$
|\dot{z}|\left(\left[t_{1}, t_{2}\right] \times C\right):=\int_{C} \mathrm{R}_{1}\left(z\left(t_{2}\right)-z\left(t_{1}\right)\right) \mathrm{d} x,
$$

and, for simplicity, we shall write $\int_{0}^{t} \int_{\Omega} \eta|\dot{z}| \mathrm{d} x \mathrm{~d} s$ instead of $\iint_{[0, t] \times \Omega} \eta|\dot{z}|(\mathrm{d} s \mathrm{~d} x)$.

Since $z$ has at most BV-regularity as a function of time, it may have (at most countably many) jump points, where the left and right limits $z\left(t_{-}\right), z\left(t_{+}\right) \in L^{1}(\Omega)$ differ. Indeed, from $z \in L^{\infty}\left(0, T ; W^{1, q}(\Omega)\right) \cap \mathrm{BV}\left([0, T] ; L^{1}(\Omega)\right)$ it is immediate to deduce that, at every $t \in[0, T]$ (with the standard conventions $z\left(0_{-}\right):=z(0)$ and $z\left(T_{+}\right):=z(T)$ ), both $z\left(t_{-}\right)$ and $z\left(t_{+}\right)$are elements in $W^{1, q}(\Omega)$, with $z\left(t_{-}\right)=\lim _{s \uparrow t} z(s)$ and $z\left(t_{+}\right)=\lim _{s \downarrow t} z(s)$ w.r.t. the weak topology of $W^{1, q}(\Omega)$. In particular, the right limit $z\left(0_{+}\right)$exists, and it may be $z\left(0_{+}\right) \neq z(0)=z_{0}$ (observe that, by (2.7) the initial condition is fulfilled as an equality in $W^{1, q}(\Omega)$ ). In that case, the mechanical energy balance $(2.12 \mathrm{c})$ records the jump of the stored/dissipated energies at the initial time.

Remark 2.4 (Total energy balance) Summing up the mechanical energy inequality (2.12c) and the weak heat equation (2.12d) tested by $\eta \equiv 1$, yields the total energy balance

$$
\begin{aligned}
& \int_{\Omega} \frac{\rho}{2}|\dot{u}(t)|^{2} \mathrm{~d} x+\mathcal{E}(t, u(t), z(t))+\int_{\Omega} \theta(t) \mathrm{d} x=\int_{\Omega} \frac{\rho}{2}\left|\dot{u}_{0}\right|^{2} \mathrm{~d} x+\mathcal{E}\left(0, u_{0}, z_{0}\right)+\int_{\Omega} \theta_{0} \mathrm{~d} x \\
& +\int_{0}^{t} \partial_{t} \mathcal{E}(s, u(s), z(s)) \mathrm{d} s+\int_{0}^{t} \int_{\Omega} H \mathrm{~d} x \mathrm{~d} s+\int_{0}^{t} \int_{\partial \Omega} h \mathrm{~d} \mathcal{H}^{d-1}(x) \mathrm{d} s .
\end{aligned}
$$

Remark 2.5 (Improved regularity on $\ddot{u}$ ) From the definition of energetic solution we can gain improved regularity for the time derivatives of the displacement. Indeed, let $(u, z, \theta)$ be as 
in (2.11) and such that the weak momentum equation (2.12b) holds. Then (1.1a) holds in the sense of distributions and

$$
\begin{aligned}
\rho\|\ddot{u}\|_{L^{2}\left(0, T ; H_{\mathrm{D}}^{1}\left(\Omega ; \mathbb{R}^{d}\right)^{*}\right)}= & \sup _{\|v\| \leq 1} \int_{0}^{T} \int_{\Omega}(\mathbb{D}(z, \theta) e(\dot{u})+\mathbb{C}(z) e(u)-\theta \mathbb{B}): e(v) \mathrm{d} x \mathrm{~d} t \\
& -\int_{0}^{T}\langle f, v\rangle_{H_{\mathrm{D}}^{1}\left(\Omega ; \mathbb{R}^{d}\right)} \mathrm{d} t,
\end{aligned}
$$

where the supremum is taken over all functions such that $\|v\|_{L^{2}\left(0, T ; H_{\mathrm{D}}^{1}\left(\Omega ; \mathbb{R}^{d}\right)\right)} \leq 1$. The lefthand side of the previous equality is uniformly bounded thanks to (2.3), (2.9), and (2.11), thus we deduce that $\ddot{u} \in L^{2}\left(0, T ; H_{\mathrm{D}}^{1}\left(\Omega ; \mathbb{R}^{d}\right)^{*}\right)$. Since the spaces $H_{\mathrm{D}}^{1}\left(\Omega ; \mathbb{R}^{d}\right) \subset L^{2}\left(\Omega ; \mathbb{R}^{d}\right) \subset$ $H_{\mathrm{D}}^{1}\left(\Omega ; \mathbb{R}^{d}\right)^{*}$ form a Gelfand triple, in view of e.g. [36, Chapter 1, Sec. 2.4, Proposition 2.2], we conclude that

$$
\begin{aligned}
& \int_{t_{1}}^{t_{2}}\langle\ddot{u}, \dot{u}\rangle_{H_{\mathrm{D}}^{1}\left(\Omega ; \mathbb{R}^{d}\right)} \mathrm{d} t \\
& \quad=\frac{1}{2}\left\langle\dot{u}\left(t_{2}\right), \dot{u}\left(t_{2}\right)\right\rangle_{H_{\mathrm{D}}^{1}\left(\Omega ; \mathbb{R}^{d}\right)}-\frac{1}{2}\left\langle\dot{u}\left(t_{1}\right), \dot{u}\left(t_{1}\right)\right\rangle_{H_{\mathrm{D}}^{1}\left(\Omega ; \mathbb{R}^{d}\right)} \\
& \quad=\frac{1}{2}\left\|\dot{u}\left(t_{2}\right)\right\|_{L^{2}\left(\Omega ; \mathbb{R}^{d}\right)}^{2}-\frac{1}{2}\left\|\dot{u}\left(t_{1}\right)\right\|_{L^{2}\left(\Omega ; \mathbb{R}^{d}\right)}^{2}
\end{aligned}
$$

for every $t_{1}, t_{2} \in[0, T]$. Hence, $\dot{u}$ can be used as a test function in (2.12b).

We are now in a position to state the main result of this paper. The last part of the assertion concerns the strict positivity of the absolute temperature $\theta$. In particular, under (2.15) below we are able to specify, in terms of the given data, the constant which bounds $\theta$ from below.

Theorem 2.6 (Existence of energetic solutions (2.11)-(2.12)) Under assumptions (2.1)(2.4), (2.5), and (2.6), and (2.8) on the data $f_{\mathrm{V}}, f_{\mathrm{S}}, H$, and $h$, for every quadruple $\left(u_{0}, \dot{u}_{0}, z_{0}, \theta_{0}\right)$ fulfilling (2.7) with $z_{0}$ satisfying (2.12a), there exists an energetic solution $(u, z, \theta)$ to the Cauchy problem for system (1.1).

Moreover, there exists $\widetilde{\theta}>0$ such that

$$
\theta(t, x) \geq \tilde{\theta}>0 \quad \text { for a.a. }(t, x) \in(0, T) \times \Omega .
$$

Furthermore, if in addition

$$
\begin{aligned}
& \exists H_{*}>0: H(t, x) \geq H_{*} \text { for a.a. }(t, x) \in(0, T) \times \Omega \\
& \quad \text { and } \theta_{0}(x) \geq \sqrt{H_{*} / \bar{c}} \text { for a.a. } x \in \Omega,
\end{aligned}
$$

where $\bar{c}:=\frac{\left(C_{\mathbb{B}}\right)^{2}}{2 C_{\mathbb{D}}^{1}}$, then

$$
\theta(t, x) \geq \max \left\{\tilde{\theta}, \sqrt{H_{*} / \bar{c}}\right\} \text { for a.a. }(t, x) \in(0, T) \times \Omega .
$$

The proof of Theorem 2.6 will be developed in Sects. 3 and 4 by time-discretization (see Propositions 4.1-4.2).

Remark 2.7 (Time-dependent Dirichlet loadings) The existence of energetic solutions can be proven also when time-dependent Dirichlet loadings are considered for the displacement $u$ instead of the homogeneous Dirichlet condition (1.3), in the case the viscous tensor $\mathbb{D}$ is independent of $z$ and $\theta$. This restriction is due to technical reasons, related to the derivation of suitable estimates for the approximate solutions to (1.1).

An alternative damage model, that still features a $(z, \theta)$-dependence of $\mathbb{D}$, is discussed in [38], where a time-dependent loading for $u$ can be encompassed in the analysis, albeit under suitable stronger conditions. 
Remark 2.8 (Failure of "entropic" solutions) As already mentioned, the regularity for the temperature $\theta \in L^{2}\left(0, T ; H^{1}(\Omega)\right) \cap \mathrm{BV}\left([0, T] ; W^{2, d+\delta}(\Omega)^{*}\right)$ results from careful estimates on the heat equation $(1.1 \mathrm{c})$, tailored on the quadratic character of its right-hand side and drawn from [23]. There, the analysis of the full system for phase transitions proposed by Frémond [24], featuring a heat equation with an $L^{1}$ right-hand side, was carried out.

The techniques from [23] have been recently extended in [63] to analyze a model for rate-dependent damage in thermo-viscoelasticity. Namely, in place of the 1-homogeneous dissipation potential $R_{1}$ from (1.2), the flow rule for the damage parameter in [63] features the quadratic dissipation $\mathrm{R}_{2}(\dot{z})=\frac{1}{2}|\dot{z}|^{2}$ if $\dot{z} \leq 0$, and $\mathrm{R}_{2}(\dot{z})=\infty$ else. Consequently, the heat equation in [63] is of the type

$$
\dot{\theta}-\operatorname{div}(\mathbb{K}(z, \theta) \nabla \theta)=|\dot{z}|^{2}+\mathbb{D}(z) e(\dot{u}): e(\dot{u})-\theta \mathbb{B}: e(\dot{u})+H \quad \text { in }(0, T) \times \Omega .
$$

In [63], under a weaker growth condition on $\mathbb{K}$ than the present (2.6), it was possible to prove an existence result for a weaker formulation of (2.17), consisting of an entropy inequality and of a total energy inequality. The resulting notion of "entropic" solution, originally proposed in [23], indeed reflects the strict positivity of the temperature, and the fact that the entropy increases along solutions. Without going into details, let us mention that this entropy inequality is (formally) obtained by testing (2.17) by $\varphi \theta^{-1}$, with $\varphi$ a smooth test function, and integrating in time. This procedure is fully justified because $\theta$ can be shown to be bounded away from zero by a positive constant, hence $\varphi(t) \theta^{-1}(t) \in L^{\infty}(\Omega)$ for almost all $t \in(0, T)$, and the integrals $\int_{0}^{T} \int_{\Omega}|\dot{z}|^{2} \varphi \theta^{-1} \mathrm{~d} x \mathrm{~d} t$ and $\int_{0}^{T} \int_{\Omega} \mathbb{D}(z) e(\dot{u}): e(\dot{u}) \varphi \theta^{-1} \mathrm{~d} x \mathrm{~d} t$ resulting from the first and second terms on the right-hand side of (2.17) are well-defined.

In the present rate-independent context, proving an existence result for the entropic formulation of (1.1c) seems to be out of reach. Indeed, in such formulation the term $\int_{0}^{T} \int_{\Omega}|\dot{z}|^{2} \varphi \theta^{-1} \mathrm{~d} x \mathrm{~d} t$ would have to be replaced by $\int_{[0, T] \times \Omega} \varphi \theta^{-1}|\dot{z}|(\mathrm{d} x \mathrm{~d} t)$, with $|\dot{z}|$ the total variation measure of $z, \mathrm{cf}$. (2.12d), but the above integral is not well defined since $\varphi \theta^{-1}$ is not a continuous function.

\section{Time-Discretization}

\subsection{The Time-Discrete Scheme}

Given a partition

$$
0=t_{n}^{0}<\cdots<t_{n}^{n}=T \quad \text { with } \quad t_{n}^{k}-t_{n}^{k-1}=\frac{T}{n}=: \tau_{n},
$$

we construct a family of discrete solutions $\left(u_{n}^{k}, z_{n}^{k}, \theta_{n}^{k}\right)_{k=1, \ldots, n}$ by solving recursively the time-discretization scheme (3.3) below, where the data $f, H$, and $h$ are approximated by local means as follows

$$
f_{n}^{k}:=\frac{1}{\tau_{n}} \int_{t_{n}^{k-1}}^{t_{n}^{k}} f(s) \mathrm{d} s, \quad H_{n}^{k}:=\frac{1}{\tau_{n}} \int_{t_{n}^{k-1}}^{t_{n}^{k}} H(s) \mathrm{d} s, \quad h_{n}^{k}:=\frac{1}{\tau_{n}} \int_{t_{n}^{k-1}}^{t_{n}^{k}} h(s) \mathrm{d} s,
$$

and the above integrals need to be understood in the Bochner sense.

We mention in advance that we have to add the regularizing term $-\tau_{n} \operatorname{div}\left(\left|e\left(u_{n}^{k}\right)\right|^{\gamma-2} e\left(u_{n}^{k}\right)\right)$ in the discrete momentum equation, with $\gamma>4$. Basically, the reason for this is that we need to compensate the quadratic term in $e\left(u_{n}^{k}\right)$ on the right-hand side of the discrete heat equation (3.3c). In practice, the term $-\tau_{n} \operatorname{div}\left(\left|e\left(u_{n}^{k}\right)\right|^{\gamma-2} e\left(u_{n}^{k}\right)\right)$ will have a key role in proving that the pseudomonotone operator in terms of which the (approximate) discrete system can be 
506

reformulated is coercive, and thus such system admits solutions. Because of this additional regularization, it will be necessary to further approximate the initial datum $u_{0}$ from (2.7a) by a sequence (cf. [10, p. 56, Corollary 2])

$$
\left(u_{n}^{0}\right)_{n} \subset W_{\mathrm{D}}^{1, \gamma}\left(\Omega ; \mathbb{R}^{d}\right) \text { such that } u_{n}^{0} \rightarrow u_{0} \text { in } H_{\mathrm{D}}^{1}\left(\Omega ; \mathbb{R}^{d}\right) \text { as } n \rightarrow \infty,
$$

where $W_{\mathrm{D}}^{1, \gamma}\left(\Omega ; \mathbb{R}^{d}\right)=\left\{v \in W^{1, \gamma}\left(\Omega ; \mathbb{R}^{d}\right): v=0\right.$ on $\partial_{\mathrm{D}} \Omega$ in the trace sense $\}$.

For the weak formulation of the discrete heat equation, we also need to introduce the function space appropriate for $\theta$, dependent on a given $\bar{z} \in L^{\infty}(\Omega)$

$$
X_{\bar{z}}:=\left\{\vartheta \in H^{1}(\Omega): \int_{\Omega} \mathbb{K}(\bar{z}, \vartheta) \nabla \vartheta \cdot \nabla v \mathrm{~d} x \text { is well defined for all } v \in H^{1}(\Omega)\right\} .
$$

In fact, the above space encodes the sharpest property that we will be able to obtain for our discrete solutions $\left(u_{n}^{k}, z_{n}^{k}, \theta_{n}^{k}\right)_{k=1}^{n}$. This will be proven by approximating system (3.3) by truncations, so that in the truncated system the heat equation is standardly weakly formulated in $H^{1}(\Omega)^{*}$. Passing to the limit as the truncation parameter tends to infinity, with a careful comparison argument in the discrete heat equation (cf. the proof of [63, Lemma 4.4] for all details), it is possible to prove that $\theta_{n}^{k} \in X_{z_{n}^{k}}$.

We consider the following weakly-coupled discretization scheme (in fact, only the momentum and the heat equation are coupled, while the discrete equation for $z$ is decoupled from them):

\section{Problem 3.1 Starting from}

$$
u_{n}^{0}, \quad z_{n}^{0}:=z_{0}, \quad \theta_{n}^{0}:=\theta_{0},
$$

and setting $u_{n}^{-1}:=u_{n}^{0}-\tau_{n} \dot{u}_{0}$, find $\left(u_{n}^{k}, z_{n}^{k}, \theta_{n}^{k}\right)_{k=1}^{n} \subset W_{\mathrm{D}}^{1, \gamma}\left(\Omega ; \mathbb{R}^{d}\right) \times W^{1, q}(\Omega) \times X_{z_{n}^{k}}$ such that the following hold:

- Minimality of $z_{n}^{k}$ :

$$
z_{n}^{k} \in \operatorname{argmin}\left\{\mathcal{R}_{1}\left(z-z_{n}^{k-1}\right)+\mathcal{E}\left(t_{n}^{k}, u_{n}^{k-1}, z\right): z \in \mathcal{Z}\right\} ;
$$

- Time-discrete weak formulation of the coupled momentum balance and the heat equation: Find $u_{n}^{k} \in W_{\mathrm{D}}^{1, \gamma}\left(\Omega ; \mathbb{R}^{d}\right)$ and $\theta_{n}^{k} \in X_{z_{n}^{k}}$ such that

$\rho \int_{\Omega} \frac{u_{n}^{k}-2 u_{n}^{k-1}+u_{n}^{k-2}}{\tau_{n}^{2}} \cdot v \mathrm{~d} x$

$$
\begin{aligned}
& +\int_{\Omega}\left(\mathbb{D}\left(z_{n}^{k-1}, \theta_{n}^{k-1}\right) e\left(\frac{u_{n}^{k}-u_{n}^{k-1}}{\tau_{n}}\right)+\mathbb{C}\left(z_{n}^{k}\right) e\left(u_{n}^{k}\right)-\theta_{n}^{k} \mathbb{B}+\tau_{n}\left|e\left(u_{n}^{k}\right)\right|^{\gamma-2} e\left(u_{n}^{k}\right)\right): e(v) \mathrm{d} x \\
= & \left\langle f_{n}^{k}, v\right\rangle_{H_{\mathrm{D}}^{1}\left(\Omega ; \mathbb{R}^{d}\right)} \quad \text { for all } v \in W_{\mathrm{D}}^{1, \gamma}\left(\Omega ; \mathbb{R}^{d}\right),
\end{aligned}
$$

$$
\begin{aligned}
& \int_{\Omega} \frac{\theta_{n}^{k}-\theta_{n}^{k-1}}{\tau_{n}} \eta \mathrm{d} x+\int_{\Omega} \mathbb{K}\left(z_{n}^{k}, \theta_{n}^{k}\right) \nabla \theta_{n}^{k} \cdot \nabla \eta \mathrm{d} x \\
& =\int_{\Omega} \frac{z_{n}^{k-1}-z_{n}^{k}}{\tau_{n}} \eta \mathrm{d} x+\int_{\Omega}\left(\mathbb{D}\left(z_{n}^{k-1}, \theta_{n}^{k-1}\right) e\left(\frac{u_{n}^{k}-u_{n}^{k-1}}{\tau_{n}}\right)-\theta_{n}^{k} \mathbb{B}\right): e\left(\frac{u_{n}^{k}-u_{n}^{k-1}}{\tau_{n}}\right) \eta \mathrm{d} x \\
& +\int_{\partial \Omega} h_{n}^{k} \eta \mathrm{d} \mathcal{H}^{d-1}(x)+\left\langle H_{n}^{k}, \eta\right\rangle_{H^{1}(\Omega)} \quad \text { for all } \eta \in H^{1}(\Omega) .
\end{aligned}
$$


The above time-discrete problem has been carefully designed in such a way as to be weakly-coupled in that, for each $k \in\{1, \ldots, n\}$, it can be solved successively starting from (3.3a) and then solving the system (3.3b)-(3.3c). See [63, Remark 4.3] for similar ideas.

Our existence result for Problem 3.1 reads:

Proposition 3.2 Let the assumptions of Theorem 2.6 hold true. Then there exists a solution

$$
\left(u_{n}^{k}, z_{n}^{k}, \theta_{n}^{k}\right)_{k=1}^{n} \subset W_{\mathrm{D}}^{1, \gamma}\left(\Omega ; \mathbb{R}^{d}\right) \times W^{1, q}(\Omega) \times H^{1}(\Omega)
$$

to Problem 3.1, satisfying the following properties: There exists $\tilde{\theta}>0$ such that

$$
\theta_{n}^{k} \geq \tilde{\theta}>0 \quad \text { for all } k=1, \ldots, n, \quad \text { for all } n \in \mathbb{N} .
$$

Furthermore, if in addition (2.15) holds, then

$$
\theta_{n}^{k} \geq \max \left\{\tilde{\theta}, \sqrt{H_{*} / \bar{c}}\right\}>0 \quad \text { for all } k=1, \ldots, n, \text { for all } n \in \mathbb{N},
$$

with $H_{*}$ and $\bar{c}$ from (2.15).

While the existence of solutions for (3.3a) follows from the direct method of the calculus of variations in a straightforward manner, the existence proof for system (3.3b)-(3.3c) is more involved, due to the quasilinear character of the discrete heat equation. This is due to the fact that the viscous dissipation $\mathbb{D}\left(z_{n}^{k-1}, \theta_{n}^{k-1}\right) e\left(\frac{u_{n}^{k}-u_{n}^{k-1}}{\tau_{n}}\right): e\left(\frac{u_{n}^{k}-u_{n}^{k-1}}{\tau_{n}}\right)$ as well as the thermal stresses $\theta_{n}^{k-1} \mathbb{B}: e\left(\frac{u_{n}^{k}-u_{n}^{k-1}}{\tau_{n}}\right)$ only happen to be of $L^{1}$-summability as a consequence of (3.3b). Observe in particular that $\mathbb{C}\left(z_{n}^{k}\right), \mathbb{D}\left(z_{n}^{k-1}, \theta_{n}^{k-1}\right) \in\left(L^{\infty}(\Omega) \cap W^{1, q}(\Omega)\right)^{d \times d \times d \times d}$, and we do not impose the assumption $q>d$, which would guarantee the continuity of the coefficients. As it is demonstrated by the counterexample in [54], in absence of continuous coefficients, it is not ensured that the solution of (3.3b) enjoys elliptic regularity. Because of this expected lack of additional regularity, the existence of solutions for the coupled system (3.3b)-(3.3c) will be verified by means of an approximation procedure, in which the $L^{1}$ righthand side in (3.3c) is replaced by a sequence of truncations. For this we proceed along the lines of [63] where the analysis of a time-discrete system analogous to (3.3a)-(3.3c) was carried out. The existence of solutions to the approximate discrete system in turn follows from an existence result for a wide class of elliptic equations, in the framework of the Leray-Schauder theory of pseudo-monotone operators. We will then conclude the existence of solutions to (3.3b)-(3.3c) by passing to the limit with the truncation parameter. In such a step, we shall exploit the strict positivity of the approximate discrete temperatures, cf. (3.15) below. This property and the convergence of the approximate discrete temperatures clearly imply the strict positivity (3.4). Arguing directly on the non-truncated discrete heat equation, we will also obtain the enhanced positivity property (3.5) which, unlike (3.13), in fact provides a tunable threshold from below to the discrete temperatures.

In the forthcoming proof, we will use that for any convex (differentiable) function $\psi$ : $\mathbb{R} \rightarrow(-\infty,+\infty]$

$$
\psi(x)-\psi(y) \leq \psi^{\prime}(x)(x-y) \text { for all } x, y \in \operatorname{dom}(\psi) .
$$

Proof Existence of a minimizer to (3.3a): We first verify the coercivity of the functional $z \mapsto \mathcal{E}\left(t_{n}^{k}, u_{n}^{k-1}, z\right)+\mathcal{R}_{1}\left(z-z_{n}^{k-1}\right): W^{1, q}(\Omega) \rightarrow \mathbb{R} \cup\{\infty\}$, where $\mathcal{R}_{1}$ is the dissipation potential (2.10). Indeed, by the positivity of $\mathcal{R}_{1}(\cdot)$ and assumption $(2.5 \mathrm{~d})$ on the density $G$ we have

$\mathcal{E}\left(t_{n}^{k}, u_{n}^{k-1}, z\right)+\mathcal{R}_{1}\left(z-z_{n}^{k-1}\right) \geq \int_{\Omega} G(z, \nabla z) \mathrm{d} x-C \geq C_{G}^{1}\|z\|_{W^{1, q}(\Omega)}^{q}-C_{G}^{1} \mathcal{L}^{d}(\Omega)-C$, 
where we also used that $G(z(x), \nabla z(x))<\infty$ implies $z(x) \in[0,1]$, cf. (2.5a). By the convexity and the continuity assumptions $(2.5 \mathrm{~b})-(2.5 \mathrm{c})$ on $G$ and by the properties of $\mathcal{R}_{1}$ we conclude that the functional

$$
\mathcal{E}\left(t_{n}^{k}, u_{n}^{k-1}, \cdot\right)+\mathcal{R}_{1}\left((\cdot)-z_{n}^{k-1}\right): W^{1, q}(\Omega) \rightarrow \mathbb{R} \cup\{\infty\}
$$

is weakly sequentially lower semicontinuous. Since $z=\left\{z \in W^{1, q}(\Omega): z \in\right.$ $[0,1]$ a.e. in $\Omega\}$, see $(2.2)$, is a closed subset of a reflexive Banach space, the direct method of the calculus of variations ensures the existence of a minimizer $z_{n}^{k} \in Z$.

Existence of an approximate solution to system (3.3b)-(3.3c): As in [63, proof of Lemma 4.4], we approximate (3.3b)-(3.3c) by a suitable truncation of the heat conductivity matrix $\mathbb{K}$, in such a way as to reduce to an elliptic operator with bounded coefficients in the discrete heat equation. In a similar manner we treat the $L^{1}$ right-hand sides in order to improve their integrability. Accordingly, we truncate all occurrences of $\theta_{n}^{k}$ in the respective terms of system (3.3b)-(3.3c). We show that the approximate system thus obtained admits solutions by resorting to an existence result from the theory of elliptic systems featuring pseudo-monotone operators drawn from [60]. Hence, we pass to the limit with the truncation parameter and conclude the existence of solutions to (3.3b)-(3.3c).

Let $z_{n}^{k}$ be a solution of (3.3a). In what follows, we shall denote by $\overline{\mathbb{K}}=\overline{\mathbb{K}}(x, \theta)$ the function $\mathbb{K}\left(z_{n}^{k}(x), \theta\right)$. Let $M>0$. We introduce the truncation operator

$$
\mathcal{T}_{M}(\theta):= \begin{cases}0 & \text { if } \theta<0, \\ \theta & \text { if } 0 \leq \theta \leq M, \\ M & \text { if } \theta>M,\end{cases}
$$

and we set

$$
\overline{\mathbb{K}}_{M}: \Omega \times \mathbb{R} \rightarrow \mathbb{R}^{d \times d}, \quad \overline{\mathbb{K}}_{M}(x, \theta):=\overline{\mathbb{K}}\left(x, \mathcal{T}_{M}(\theta)\right) .
$$

Since $\mathbb{K} \in C^{0}\left(\mathbb{R} \times \mathbb{R} ; \mathbb{R}^{d \times d}\right)$ and $0 \leq z_{n}^{k}(x) \leq 1$ for almost all $x \in \Omega$, it is immediate to check that there exists a positive constant $C_{M}$ such that $\left|\overline{\mathbb{K}}_{M}(x, \theta)\right| \leq C_{M}$ for almost all $x \in \Omega$ and $\theta \in \mathbb{R}$. The truncated version of system (3.3b)-(3.3c) thus reads: find $(u, \theta) \in$ $W_{\mathrm{D}}^{1, \gamma}\left(\Omega ; \mathbb{R}^{d}\right) \times H^{1}(\Omega)$ such that

$$
\begin{aligned}
& \rho \int_{\Omega} \frac{u-2 u_{n}^{k-1}+u_{n}^{k-2}}{\tau_{n}^{2}} \cdot v \mathrm{~d} x+\int_{\Omega}\left(\mathbb{D}\left(z_{n}^{k-1}, \theta_{n}^{k-1}\right) e\left(\frac{u-u_{n}^{k-1}}{\tau_{n}}\right)+\mathbb{C}\left(z_{n}^{k}\right) e(u)\right. \\
& \left.-\mathcal{T}_{M}(\theta) \mathbb{B}+\tau_{n}|e(u)|^{\gamma-2} e(u)\right): e(v) \mathrm{d} x \\
& =\left\langle f_{n}^{k}, v\right\rangle_{H_{\mathrm{D}}^{1}\left(\Omega ; \mathbb{R}^{d}\right)}
\end{aligned}
$$

$$
\begin{array}{lr}
\int_{\Omega} \frac{\theta-\theta_{n}^{k-1}}{\tau_{n}} \eta \mathrm{d} x+\int_{\Omega} \overline{\mathbb{K}}_{M}(x, \theta) \nabla \theta \cdot \nabla \eta \mathrm{d} x & \\
=\int_{\Omega} \frac{z_{n}^{k-1}-z_{n}^{k}}{\tau_{n}} \eta \mathrm{d} x+\int_{\Omega}\left(\mathbb{D}\left(z_{n}^{k-1}, \theta_{n}^{k-1}\right) e\left(\frac{u-u_{n}^{k-1}}{\tau_{n}}\right)-\mathcal{T}_{M}(\theta) \mathbb{B}\right): e\left(\frac{u-u_{n}^{k-1}}{\tau_{n}}\right) \eta \mathrm{d} x \\
+\int_{\partial \Omega} h_{n}^{k} \eta \mathrm{d} \mathcal{H}^{d-1}(x)+\left\langle H_{n}^{k}, \eta\right\rangle_{H^{1}(\Omega)} & \text { for all } \eta \in H^{1}(\Omega) .
\end{array}
$$




$$
\begin{aligned}
& \rho \int_{\Omega} u \cdot v \mathrm{~d} x \\
& \quad+\tau_{n} \int_{\Omega}\left(\mathbb{D}\left(z_{n}^{k-1}, \theta_{n}^{k-1}\right) e(u)+\tau_{n} \mathbb{C}\left(z_{n}^{k}\right) e(u)-\tau_{n} \mathcal{T}_{M}(\theta) \mathbb{B}+\tau_{n}^{2}|e(u)|^{\gamma-2} e(u)\right): e(v) \mathrm{d} x \\
& =\rho \int_{\Omega}\left(2 u_{n}^{k-1}-u_{n}^{k-2}\right) \cdot v \mathrm{~d} x+\tau_{n} \int_{\Omega} \mathbb{D}\left(z_{n}^{k-1}, \theta_{n}^{k-1}\right) e\left(u_{n}^{k-1}\right): e(v) \mathrm{d} x+\tau_{n}^{2}\left\langle f_{n}^{k}, v\right\rangle_{H_{\mathrm{D}}^{1}\left(\Omega ; \mathbb{R}^{d}\right)} \\
& \quad \text { for all } v \in W_{\mathrm{D}}^{1, \gamma}\left(\Omega ; \mathbb{R}^{d}\right),
\end{aligned}
$$

$$
\begin{aligned}
\int_{\Omega} \theta & \eta \mathrm{d} x+\tau_{n} \int_{\Omega} \overline{\mathbb{K}}_{M}(x, \theta) \nabla \theta \cdot \nabla \eta \mathrm{d} x-\frac{1}{\tau_{n}} \int_{\Omega} \mathbb{D}\left(z_{n}^{k-1}, \theta_{n}^{k-1}\right) e(u): e(u) \eta \mathrm{d} x \\
& +\int_{\Omega} \mathcal{T}_{M}(\theta) \mathbb{B}: e(u) \eta \mathrm{d} x+\frac{2}{\tau_{n}} \int_{\Omega} \mathbb{D}\left(z_{n}^{k-1}, \theta_{n}^{k-1}\right) e(u): e\left(u_{n}^{k-1}\right) \eta \mathrm{d} x \\
& -\int_{\Omega} \mathcal{T}_{M}(\theta) \mathbb{B}: e\left(u_{n}^{k-1}\right) \eta \mathrm{d} x \\
= & \int_{\Omega} \theta_{n}^{k-1} \eta \mathrm{d} x+\frac{1}{\tau_{n}} \int_{\Omega} \mathbb{D}\left(z_{n}^{k-1}, \theta_{n}^{k-1}\right) e\left(u_{n}^{k-1}\right): e\left(u_{n}^{k-1}\right) \eta \mathrm{d} x \\
& +\int_{\Omega}\left(z_{n}^{k-1}-z_{n}^{k}\right) \eta \mathrm{d} x+\tau_{n} \int_{\partial \Omega} h_{n}^{k} \eta \mathrm{d} \mathcal{H}^{d-1}(x)+\tau_{n}\left\langle H_{n}^{k}, \eta\right\rangle_{H^{1}(\Omega)} \quad \text { for all } \eta \in H^{1}(\Omega),
\end{aligned}
$$

which in turn can be recast in the form

$$
\mathcal{A}_{k, M}(u, \theta)=B_{k-1} .
$$

Here, $\mathcal{A}_{k, M}: W_{\mathrm{D}}^{1, \gamma}\left(\Omega ; \mathbb{R}^{d}\right) \times H^{1}(\Omega) \rightarrow W_{\mathrm{D}}^{1, \gamma}\left(\Omega ; \mathbb{R}^{d}\right)^{*} \times H^{1}(\Omega)^{*}$ is the elliptic operator, acting on the unknown $(u, \theta)$, defined by the left-hand sides of (3.8a) and (3.8b), while $B_{k-1}$ is the vector defined by the right-hand side terms in system (3.8). It can be verified that $\mathcal{A}_{k, M}$ is a pseudo-monotone operator in the sense of [60, Chapter II, Definition 2.1]: without entering into details, we may in fact observe that $\mathcal{A}_{k, M}$ is given by the sum of either bounded, radially continuous, monotone operators, or totally continuous operators, cf. [60, Chapter II, Definition 2.3, Lemma 2.9, Cor. 2.12]. Furthermore, crucially exploiting the presence of the regularizing term $-\tau_{n} \operatorname{div}\left(|e(u)|^{\gamma-2} e(u)\right)$, with $\gamma>4$, in the discrete momentum balance, we may show that $\mathcal{A}_{k, M}$ is coercive on $W_{\mathrm{D}}^{1, \gamma}\left(\Omega ; \mathbb{R}^{d}\right) \times H^{1}(\Omega)$. This can be checked directly on system (3.8), testing (3.8a) by $u$ and (3.8b) by $\theta$ and adding the resulting equations: it is then sufficient to deduce from these calculations an estimate for $\|u\|_{W_{\mathrm{D}}^{1, \gamma}\left(\Omega ; \mathbb{R}^{d}\right)}$ and $\|\theta\|_{H^{1}(\Omega)}$. We refer to [63, proof of Lemma 4.4] for all the detailed calculations, which show that, since $\gamma>4$, the term $-\tau_{n} \operatorname{div}\left(|e(u)|^{\gamma-2} e(u)\right)$ can absorb the quadratic terms in $e(u)$ on the righthand side of (3.7b). In this way, it is possible to carry out the test of (3.8b) by $\theta$ and obtain the bound for $\|\theta\|_{H^{1}(\Omega)}$ : for this, one also exploits that the operator with coefficients $\overline{\mathbb{K}}_{M}$ is uniformly elliptic thanks to (2.6b). Since $\mathcal{A}_{k, M}$ is pseudo-monotone and coercive, we are in a position to apply [60, Chapter II, Theorem 2.6] to system (3.8), for every $M \in \mathbb{N}$ thus deducing the existence of a solution $(u, \theta)$ which shall be hereafter denoted as $\left(u_{n, M}^{k}, \theta_{n, M}^{k}\right)$.

Positivity of $\theta_{n, M}^{k}$ : First of all, we show that $\theta_{n, M}^{k} \geq 0$ a.e. in $\Omega$. To this end, we test the (approximate) discrete heat equation (3.7b) by $-\left(\theta_{n, M}^{k}\right)^{-}=\min \left\{\theta_{n, M}^{k}, 0\right\}$. We thus obtain 


$$
\begin{aligned}
\int_{\Omega} & \frac{1}{\tau_{n}}\left|\left(\theta_{n, M}^{k}\right)^{-}\right|^{2} \mathrm{~d} x+\int_{\Omega} \frac{1}{\tau_{n}} \theta_{n}^{k-1}\left(\theta_{n, M}^{k}\right)^{-} \mathrm{d} x+\int_{\Omega} \overline{\mathbb{K}}_{M}\left(x, \theta_{n, M}^{k}\right) \nabla\left(\theta_{n, M}^{k}\right)^{-} \cdot \nabla\left(\theta_{n, M}^{k}\right)^{-} \mathrm{d} x \\
= & -\int_{\Omega} \frac{z_{n}^{k-1}-z_{n}^{k}}{\tau_{n}} \theta_{n, M}^{k} \mathrm{~d} x-\int_{\Omega} \mathbb{D}\left(z_{n}^{k-1}, \theta_{n}^{k-1}\right) e\left(\frac{u-u_{n}^{k-1}}{\tau_{n}}\right): e\left(\frac{u-u_{n}^{k-1}}{\tau_{n}}\right) \theta_{n, M}^{k} \mathrm{~d} x \\
& -\int_{\Omega} \mathcal{T}_{M}\left(\theta_{n, M}^{k}\right) \mathbb{B}: e\left(\frac{u-u_{n}^{k-1}}{\tau_{n}}\right) \theta_{n, M}^{k} \mathrm{~d} x+\int_{\partial \Omega} h_{n}^{k} \theta_{n, M}^{k} \mathrm{~d} \mathcal{H}^{d-1}(x)+\left\langle H_{n}^{k}, \theta_{n, M}^{k}\right\rangle_{H^{1}(\Omega)} .
\end{aligned}
$$

Now, the second term on the left-hand side is non-negative, since we may suppose, by induction, that $\theta_{n}^{k-1} \geq 0$ a.e. in $\Omega$ (in fact, for $k=0$ the strict positivity (3.4) holds with $\widetilde{\theta}=\theta_{*}$, thanks to (2.7b)). The third term is also non-negative, by ellipticity of $\overline{\mathbb{K}}_{M}$. As for the right-hand side, the first, second, fourth, and fifth terms are negative, since $z_{n}^{k-1} \geq z_{n}^{k}$ a.e. in $\Omega$, and by the positivity properties of the data $\mathbb{D}, H$, and $h$. The very definition of the truncation operator $\mathcal{T}_{M}$ does ensure that the third term is null. All in all, we conclude that $\int_{\Omega}\left|\left(\theta_{n, M}^{k}\right)^{-}\right|^{2} \mathrm{~d} x \leq 0$, whence $\left(\theta_{n, M}^{k}\right)^{-}=0$ a.e. in $\Omega$, i.e. the desired positivity. Let us now prove that $\theta_{n, M}^{k}$ fulfills (3.4), namely

$$
\theta_{n, M}^{k} \geq \tilde{\theta}>0 \text { a.e. in } \Omega \text {. }
$$

Following the lines of [63, proof of Lemma 4.4] we develop a comparison argument drawn from [23]. In this context, we will use the following estimate

$$
\mathbb{D}(\bar{z}, \bar{\theta}) \bar{e}: \bar{e}-\mathcal{T}_{M}(\bar{\theta}) \mathbb{B}: \bar{e} \geq C_{\mathbb{D}}^{1}|\bar{e}|^{2}-|\bar{e}| C_{\mathbb{B}}|\bar{\theta}| \geq \frac{C_{\mathbb{D}}^{1}}{2}|\bar{e}|^{2}-\frac{\left(C_{\mathbb{B}}\right)^{2}}{2 C_{\mathbb{D}}^{1}}|\bar{\theta}|^{2}
$$

Exploiting (3.10) and also using that $z_{k-1} \geq z_{k}$ a.e. in $\Omega$, the positivity (2.8b) of the data $H$ and $h$ and of $\theta_{n}^{k-1}$, we deduce from (3.3c) that $\theta_{n, M}^{k}$ fulfills

$$
\int_{\Omega} \theta_{n, M}^{k} \eta \mathrm{d} x+\tau_{n} \int_{\Omega} \overline{\mathbb{K}}_{M}\left(z_{n}^{k}, \theta_{n, M}^{k}\right) \nabla \theta_{n, M}^{k} \cdot \nabla \eta \mathrm{d} x \geq \int_{\Omega} \theta_{n}^{k-1} \eta \mathrm{d} x-\tau_{n} \bar{c} \int_{\Omega}\left(\theta_{n, M}^{k}\right)^{2} \eta \mathrm{d} x
$$

for all $\eta \in H^{1}(\Omega) \cap L^{\infty}(\Omega)$ with $\eta \geq 0$ a.e. in $\Omega$, with the constant $\bar{c}=\frac{\left(C_{\mathbb{B}}\right)^{2}}{2 C_{\mathbb{D}}^{1}}$ independent of $k$. Hence, we compare $\theta_{n, M}^{k}$ with the solution $v_{k} \in \mathbb{R}$ of the finite difference equation

$$
v_{k}=v_{k-1}-\tau_{n} \bar{c} v_{k}^{2}, \quad k=1, \ldots, n, \quad \text { with } v_{0}:=\theta_{*}>0 .
$$

Now, it is possible to show that

$$
v_{k} \geq \widetilde{\theta}:=\left(\bar{c} T+\frac{1}{\theta_{*}}\right)^{-1} .
$$

We test the difference of (3.11) and (3.12) by the function $L_{\varepsilon}\left(v_{k}-\theta_{n, M}^{k}\right)$, with

$$
L_{\varepsilon}(x):= \begin{cases}0 & \text { if } x \leq 0, \\ \frac{x}{\varepsilon} & \text { if } 0<x<\varepsilon, \\ 1 & \text { if } x \geq \varepsilon,\end{cases}
$$

and we conclude that

$$
\int_{\Omega}\left(v_{k}-v_{k-1}\right)-\left(\theta_{n, M}^{k}-\theta_{n}^{k-1}\right) H_{\varepsilon}\left(v_{k}-\theta_{n, M}^{k}\right) \mathrm{d} x=\tau_{n} \bar{c} \int_{\Omega}\left(\left(\theta_{n, M}^{k}\right)^{2}-v_{k}^{2}\right) H_{\varepsilon}\left(v_{k}-\theta_{n, M}^{k}\right) \mathrm{d} x \leq 0 .
$$

Observe that, in order to conclude that the above integral is negative, it was essential to preliminarily show that $\theta_{n, M}^{k} \geq 0$ a.e. in $\Omega$. Assume now that $\theta_{n}^{k-1} \geq v_{k-1}$ (which is true for 
$k=0$, cf. (2.7b)). Letting $\varepsilon \downarrow 0$ in (3.14) yields that $\theta_{n, M}^{k} \geq v_{k}$ a.e. in $\Omega$. Hence, in view of (3.13) we conclude the desired (3.9).

Passage to the limit as $M \rightarrow \infty$ : We now consider a family $\left(u_{n, M}^{k}, \theta_{n, M}^{k}\right){ }_{M}$ of solutions to the truncated system (3.7): we shall derive some a priori estimates on $\left(u_{n, M}^{k}, \theta_{n, M}^{k}\right)_{M}$ which will allow us to extract a (not relabeled) subsequence converging as $M \rightarrow \infty$ to a solution of system (3.3b)-(3.3c). For the ensuing calculations, it is crucial to observe that

$$
\exists \widetilde{\theta} \text { such that } \theta_{n, M}^{k} \geq \widetilde{\theta}>0 \text { for all } M>0 .
$$

This follows from the very same arguments as for (3.4): indeed, notice that $\widetilde{\theta}$ does not depend on $M$.

Hence, let us first test (3.7a) by $\left(u_{n, M}^{k}-u_{n}^{k-1}\right) / \tau_{n}$, (3.7b) by 1 , and add the resulting relations. Taking into account the cancelation of the coupling terms between (3.7a) and (3.7b), by convexity, cf. (3.6), we obtain

$$
\begin{aligned}
& \frac{\rho}{2 \tau_{n}^{3}} \int_{\Omega}\left|u_{n, M}^{k}-u_{n}^{k-1}\right|^{2} \mathrm{~d} x+\frac{1}{2 \tau_{n}} \int_{\Omega} \mathbb{C}\left(z_{n}^{k}\right) e\left(u_{n, M}^{k}\right): e\left(u_{n, M}^{k}\right) \mathrm{d} x \\
& \quad+\frac{1}{\gamma} \int_{\Omega}\left|e\left(u_{n, M}^{k}\right)\right|^{\gamma} \mathrm{d} x+\frac{1}{\tau_{n}} \int_{\Omega} \theta_{n, M}^{k} \mathrm{~d} x \\
& \leq \frac{\rho}{2 \tau_{n}^{3}} \int_{\Omega}\left|u_{n}^{k-1}-u_{n}^{k-2}\right|^{2} \mathrm{~d} x+\frac{1}{2 \tau_{n}} \int_{\Omega} \mathbb{C}\left(z_{n}^{k}\right) e\left(u_{n}^{k-1}\right): e\left(u_{n}^{k-1}\right) \mathrm{d} x \\
&+\frac{1}{\gamma} \int_{\Omega}\left|e\left(u_{n}^{k-1}\right)\right|^{\gamma} \mathrm{d} x+\frac{1}{\tau_{n}} \int_{\Omega} \theta_{n}^{k-1} \mathrm{~d} x \\
&+\left\langle f_{n}^{k}, \frac{u_{n, M}^{k}-u_{n}^{k-1}}{\tau_{n}}\right\rangle_{H_{\mathrm{D}}^{1}\left(\Omega ; \mathbb{R}^{d}\right)}+\int_{\Omega}\left(\frac{z_{n}^{k-1}-z_{n}^{k}}{\tau_{n}}+H_{n}^{k}\right) \mathrm{d} x \\
&+\int_{\partial \Omega} h_{n}^{k} \mathrm{~d} \mathcal{H}^{d-1}(x) \leq C_{k, n},
\end{aligned}
$$

where the constant $C_{k, n}$ is uniform with respect to the truncation parameter $M$ (but depends on $k$ and $n$ ). Therefore, also on account of (3.15) we infer that

$$
\left\|u_{n, M}^{k}\right\|_{W^{1, \gamma}\left(\Omega ; \mathbb{R}^{d}\right)}+\left\|\theta_{n, M}^{k}\right\|_{L^{1}(\Omega)} \leq C_{k, n},
$$

for a (possibly different) constant $C_{k, n}$ uniform w.r.t. $M$ but depending on $k$ and $n$. From now till the end of the discussion of the limit passage $M \rightarrow \infty$, we will omit the dependence of such constants on $k$ and $n$. As a straightforward consequence of (3.16), if we define

$$
\mathcal{S}_{M}=\left\{x \in \Omega: \theta_{n, M}^{k} \leq M\right\},
$$

using Markov's inequality, it is not difficult to infer from (3.16) that

$$
\left|\Omega \backslash S_{M}\right| \rightarrow 0 \text { as } M \rightarrow \infty .
$$

Secondly, we test (3.7b) by $\mathcal{T}_{M}\left(\theta_{n, M}^{k}\right)$. Using that

$$
\theta \mathcal{T}_{M}(\theta) \geq\left|\mathcal{T}_{M}(\theta)\right|^{2} \text { and } \overline{\mathbb{K}}_{M}(x, \theta) \nabla \theta \cdot \nabla \mathcal{T}_{M}(\theta)=\overline{\mathbb{K}}\left(x, \mathcal{T}_{M}(\theta)\right) \nabla \mathcal{T}_{M}(\theta) \cdot \nabla \mathcal{T}_{M}(\theta),
$$

we obtain

$$
\begin{aligned}
& \frac{1}{2 \tau_{n}} \int_{\Omega}\left|\mathcal{T}_{M}\left(\theta_{n, M}^{k}\right)\right|^{2} \mathrm{~d} x+\int_{\Omega} \overline{\mathbb{K}}\left(x, \mathcal{T}_{M}\left(\theta_{n, M}^{k}\right)\right) \nabla \mathcal{T}_{M}\left(\theta_{n, M}^{k}\right) \cdot \nabla \mathcal{T}_{M}\left(\theta_{n, M}^{k}\right) \mathrm{d} x \\
& \quad \leq \frac{1}{2 \tau_{n}} \int_{\Omega}\left|\theta_{n}^{k-1}\right|^{2} \mathrm{~d} x+I_{1}+I_{2}+I_{3}+I_{4}
\end{aligned}
$$


where, taking into account (2.3e) and the previously obtained (3.16), we have

$$
I_{1}:=\left|\int_{\Omega} \mathbb{D}\left(z_{n}^{k-1}, \theta_{n}^{k-1}\right) e\left(\frac{u_{n, M}^{k}-u_{n}^{k-1}}{\tau_{n}}\right): e\left(\frac{u_{n, M}^{k}-u_{n}^{k-1}}{\tau_{n}}\right) \mathcal{T}_{M}\left(\theta_{n, M}^{k}\right) \mathrm{d} x\right|
$$

$$
\leq C\left\|e\left(\frac{u_{n, M}^{k}-u_{n}^{k-1}}{\tau_{n}}\right)\right\|_{L^{4}\left(\Omega ; \mathbb{R}^{d \times d}\right)}^{4}+\frac{1}{8 \tau_{n}} \int_{\Omega}\left|\mathcal{T}_{M}\left(\theta_{n, M}^{k}\right)\right|^{2} \mathrm{~d} x,
$$

$$
\begin{aligned}
I_{2} & :=\left|\int_{\Omega} \mathcal{T}_{M}\left(\theta_{n, M}^{k}\right) \mathbb{B}: e\left(\frac{u_{n, M}^{k}-u_{n}^{k-1}}{\tau_{n}}\right) \mathcal{T}_{M}\left(\theta_{n, M}^{k}\right) \mathrm{d} x\right| \\
& \leq C\left\|e\left(\frac{u_{n, M}^{k}-u_{n}^{k-1}}{\tau_{n}}\right)\right\|_{L^{2}\left(\Omega ; \mathbb{R}^{d \times d}\right)}\left\|\mathcal{T}_{M}\left(\theta_{n, M}^{k}\right)\right\|_{L^{4}(\Omega)}^{2}
\end{aligned}
$$

$$
\leq C\left\|\mathcal{T}_{M}\left(\theta_{n, M}^{k}\right)\right\|_{L^{4}(\Omega)}^{2} \leq \frac{c_{1}}{4} \int_{\Omega}\left|\nabla \mathcal{T}_{M}\left(\theta_{n, M}^{k}\right)\right|^{2} \mathrm{~d} x+\left\|\mathcal{T}_{M}\left(\theta_{n, M}^{k}\right)\right\|_{L^{1}(\Omega)}^{2},
$$

$$
\begin{aligned}
& I_{3}:=\left|\int_{\Omega} \frac{z_{n}^{k}-z_{n}^{k-1}}{\tau_{n}} \mathcal{T}_{M}\left(\theta_{n, M}^{k}\right) \mathrm{d} x\right| \leq C+\frac{1}{8 \tau_{n}} \int_{\Omega}\left|\mathcal{T}_{M}\left(\theta_{n, M}^{k}\right)\right|^{2} \mathrm{~d} x, \\
& I_{4}:=\left|\left\langle H_{n}^{k}, \mathcal{T}_{M}\left(\theta_{n, M}^{k}\right)\right\rangle_{H^{1}(\Omega)}+\int_{\partial \Omega} h_{n}^{k} \mathcal{T}_{M}\left(\theta_{n, M}^{k}\right) \mathrm{d} \mathcal{H}^{d-1}(x)\right|
\end{aligned}
$$

$$
\leq \frac{1}{16 \tau_{n}} \int_{\Omega}\left|\mathcal{T}_{M}\left(\theta_{n, M}^{k}\right)\right|^{2} \mathrm{~d} x+\frac{c_{1}}{2} \int_{\Omega}\left|\nabla \mathcal{T}_{M}\left(\theta_{n, M}^{k}\right)\right|^{2} \mathrm{~d} x+C .
$$

where in the estimate for $I_{2}$ we have used the previously obtained bound (3.16), the Gagliardo-Nirenberg inequality $\|v\|_{L^{4}(\Omega)} \leq C\|v\|_{H^{1}(\Omega)}^{\sigma}\|v\|_{L^{1}(\Omega)}^{1-\sigma}$ for $\sigma=9 / 10$, and the Young inequality. As by (2.6b) it is $\overline{\mathbb{K}}_{M} \xi \cdot \xi \geq c_{1}|\xi|^{2}$, combining the above estimates with (3.18) and taking into account (3.16), we conclude that

$$
\left\|\mathcal{T}_{M}\left(\theta_{n, M}^{k}\right)\right\|_{L^{2}(\Omega)}+\int_{\Omega} \overline{\mathbb{K}}\left(x, \mathcal{T}_{M}\left(\theta_{n, M}^{k}\right)\right) \nabla \mathcal{T}_{M}\left(\theta_{n, M}^{k}\right) \cdot \nabla \mathcal{T}_{M}\left(\theta_{n, M}^{k}\right) \mathrm{d} x \leq C .
$$

Now, the coercivity (2.6b) implies

$$
\begin{aligned}
& \int_{\Omega} \overline{\mathbb{K}}\left(x, \mathcal{T}_{M}\left(\theta_{n, M}^{k}\right)\right) \nabla \mathcal{T}_{M}\left(\theta_{n, M}^{k}\right) \cdot \nabla \mathcal{T}_{M}\left(\theta_{n, M}^{k}\right) \mathrm{d} x \\
& \quad \geq c_{1} \int_{\Omega}\left|\mathcal{T}_{M}\left(\theta_{n, M}^{k}\right)\right|^{\kappa}\left|\nabla \mathcal{T}_{M}\left(\theta_{n, M}^{k}\right)\right|^{2} \mathrm{~d} x=c \int_{\Omega}\left|\nabla\left(\mathcal{T}_{M}\left(\theta_{n, M}^{k}\right)\right)^{(\kappa+2) / 2}\right|^{2} \mathrm{~d} x .
\end{aligned}
$$

From this, recalling the continuous embedding $H^{1} \subset L^{6}$ we infer

$$
\left\|\mathcal{T}_{M}\left(\theta_{n, M}^{k}\right)\right\|_{H^{1}(\Omega)}+\left\|\mathcal{T}_{M}\left(\theta_{n, M}^{k}\right)\right\|_{L^{3 \kappa+6}(\Omega)} \leq C .
$$

Thirdly, we test (3.7b) by $\theta_{n, M}^{k}$. Relying on estimate (3.19) to bound the second term on the right-hand side of ( $3.7 \mathrm{~b}$ ) and mimicking the above calculations, we obtain

$$
\left\|\theta_{n, M}^{k}\right\|_{H^{1}(\Omega)}+\left\|\theta_{n, M}^{k}\right\|_{L^{3 \kappa+6}\left(\mathcal{S}_{M}\right)} \leq C .
$$

With estimates (3.16), (3.19), and (3.20), combined with well-known compactness arguments, we find a pair $(u, \theta)$ such that, along a not relabeled subsequence, $\left(u_{n, M}^{k}, \theta_{n, M}^{k}\right) \rightarrow(u, \theta)$ in $W_{\mathrm{D}}^{1, \gamma}\left(\Omega ; \mathbb{R}^{d}\right) \times H^{1}(\Omega)$. The argument for passing to the limit as $M \rightarrow \infty$ in (3.7), also based on (3.17), is completely analogous to the one developed in the proof of [63, Lemma 4.4], therefore we refer to the latter paper for all details.

\section{黛 Springer}


Positivity of the discrete temperature, ad (3.4): The strict positivity (3.4) is now inherited by $\theta_{n}^{k}$ in the limit passage, as $M \rightarrow \infty$, in (3.4).

Refined positivity estimate for the discrete temperature, ad (3.5): Under the additional strict positivity (2.15) of $H$, arguing as in the above lines we infer that $\theta_{n}^{k}$ fulfills

$$
\int_{\Omega} \theta_{n}^{k} \eta \mathrm{d} x+\tau_{n} \int_{\Omega} \mathbb{K}\left(z_{n}^{k}, \theta_{n}^{k}\right) \nabla \theta_{n}^{k} \cdot \nabla \eta \mathrm{d} x \geq \int_{\Omega} \theta_{n}^{k-1} \eta \mathrm{d} x+\int_{\Omega} \tau_{n}\left(H_{*}-\bar{c}\left(\theta_{n}^{k}\right)^{2}\right) \eta \mathrm{d} x
$$

for all $\eta \in L^{\infty}(\Omega)$ with $\eta \geq 0$ a.e. in $\Omega$, with $\bar{c}>0$ the same constant as in (3.11). Hence, we compare $\theta_{n}^{k}$ with the solution $\widetilde{v}_{k} \in \mathbb{R}$

$$
\widetilde{v}_{k}=\widetilde{v}_{k-1}+\tau_{n}\left(H_{*}-\bar{c} \widetilde{v}_{k}^{2}\right), \quad k=1, \ldots, n, \quad \text { with } \widetilde{v}_{0}:=\max \left\{\theta_{*}, \sqrt{H_{*} / \bar{c}}\right\}>0,
$$

The very same arguments from [63, proof of Lemma 4.4], cf. also the previous discussion, allow us to show for all $k=0, \ldots, n$ that $\theta_{n}^{k}(x) \geq \widetilde{v}_{k}$ for almost all $x \in \Omega$. Since $\widetilde{v}_{k}>$ $\widetilde{v}_{k-1}-\tau_{n} \bar{c} \widetilde{v}_{k}^{2}$, and $\widetilde{v}_{0} \geq v_{0}=\theta_{*}$, a comparison with the solution $v_{k}$ of the finite-difference equation (3.12) and induction over $k$ yield that $\widetilde{v}_{k} \geq v_{k}$. Hence $\tilde{v}_{k} \geq \widetilde{\theta}>0$. We now aim to prove that

$$
\widetilde{v}_{k} \geq \sqrt{H_{*} / \bar{c}} \quad \text { for all } k=1, \ldots, n \text {. }
$$

We proceed by contradiction and suppose that $H_{*}>\bar{c} \widetilde{v}_{\bar{k}}^{2}$ for a certain $\bar{k} \in\{1, \ldots, n\}$. Then, we read from (3.21) that $\widetilde{v}_{\bar{k}}>\widetilde{v}_{\bar{k}-1}$. Since $\widetilde{v}_{\bar{k}-1}>0$, we then conclude that $H_{*}>\bar{c} \widetilde{v}_{\bar{k}}^{2}>$ $\bar{c} \widetilde{v}_{\bar{k}-1}^{2}$. Proceeding by induction, we thus conclude that $H_{*}>\bar{c} \widetilde{v}_{0}^{2}$, which is a contradiction to (3.21). Therefore, (3.22) ensues. This concludes the existence proof for system (3.3b)-(3.3c).

\subsection{Time-Discrete Version of the Energetic Formulation}

We now define the approximate solutions to the energetic formulation of the initial-boundary value problem for system (1.1) by suitably interpolating the discrete solutions $\left(u_{n}^{k}, z_{n}^{k}, \theta_{n}^{k}\right)_{k=1}^{n}$ from Proposition 3.2. Namely, for $t \in\left(t_{n}^{k-1}, t_{n}^{k}\right], k=1, \ldots, n$, we set

$$
\begin{array}{lll}
\bar{u}_{n}(t):=u_{n}^{k}, & \bar{\theta}_{n}(t):=\theta_{n}^{k}, & \bar{z}_{n}(t):=z_{n}^{k}, \\
\underline{u}_{n}(t):=u_{n}^{k-1}, & \underline{\theta}_{n}(t):=\theta_{n}^{k-1}, & \underline{z}_{n}(t):=z_{n}^{k-1},
\end{array}
$$

and we also consider the piecewise linear interpolants, defined by

$$
u_{n}(t):=\frac{t-t_{n}^{k-1}}{\tau_{n}} u_{n}^{k}+\frac{t_{n}^{k}-t}{\tau_{n}} u_{n}^{k-1}, \quad z_{n}(t):=\frac{t-t_{n}^{k-1}}{\tau_{n}} z_{n}^{k}+\frac{t_{n}^{k}-t}{\tau_{n}} z_{n}^{k-1}, \quad \theta_{n}(t):=\frac{t-t_{n}^{k-1}}{\tau_{n}} \theta_{n}^{k}+\frac{t_{n}^{k}-t}{\tau_{n}} \theta_{n}^{k-1} .
$$

In what follows, we shall understand the time derivative of the piecewise linear interpolant $u_{n}$ to be defined also at the nodes of the partition by

$$
\dot{u}_{n}\left(t_{n}^{k}\right):=\frac{u_{n}^{k}-u_{n}^{k-1}}{\tau_{n}}, \quad \text { for } k=1, \ldots, n .
$$

This will allow us, for instance, to state (3.27) for all $t \in[0, T]$. We also introduce the piecewise constant and linear interpolants of the discrete data $\left(f_{n}^{k}, H_{n}^{k}, h_{n}^{k}\right)_{k=1}^{n}$ in (3.1) by setting for $t \in\left(t_{n}^{k-1}, t_{n}^{k}\right]$

$$
\bar{f}_{n}(t):=f_{n}^{k}, \quad \bar{H}_{n}(t):=H_{n}^{k}, \quad \bar{h}_{n}(t):=h_{n}^{k},
$$


and $f_{n}(t):=\frac{t-t_{n}^{k-1}}{\tau_{n}} f_{n}^{k}+\frac{t_{n}^{k}-t}{\tau_{n}} f_{n}^{k-1}$ with time derivative $\dot{f}_{n}(t):=\frac{f_{n}^{k}-f_{n}^{k-1}}{\tau_{n}}$. It follows from (2.8) that, as $n \rightarrow \infty$,

$$
\begin{array}{cl}
\bar{f}_{n} \rightarrow f & \text { in } L^{p}\left(0, T ; H_{\mathrm{D}}^{1}\left(\Omega ; \mathbb{R}^{d}\right)^{*}\right) \text { for all } 1 \leq p<\infty, \\
\bar{f}_{n} \stackrel{*}{\rightarrow} f & \text { in } L^{\infty}\left(0, T ; H_{\mathrm{D}}^{1}\left(\Omega ; \mathbb{R}^{d}\right)^{*}\right), \\
\bar{f}_{n}(t) \rightarrow f(t) & \text { in } H_{\mathrm{D}}^{1}\left(\Omega ; \mathbb{R}^{d}\right)^{*} \text { for all } t \in[0, T], \\
f_{n} \rightarrow f & \text { in } H^{1}\left(0, T ; H_{\mathrm{D}}^{1}\left(\Omega ; \mathbb{R}^{d}\right)^{*}\right), \\
\bar{H}_{n} \rightarrow H & \text { in } L^{1}\left(0, T ; L^{1}(\Omega)\right) \cap L^{2}\left(0, T ; H^{1}(\Omega)^{*}\right), \\
\bar{h}_{n} \rightarrow h & \text { in } L^{1}\left(0, T ; L^{2}(\partial \Omega)\right) .
\end{array}
$$

Finally, we consider the piecewise constant interpolants associated with the partition, i.e.,

$$
\bar{\tau}_{n}(t):=t_{n}^{k} \text { and } \underline{\tau}_{n}(t):=t_{n}^{k-1} \text { for } t \in\left(t_{n}^{k-1}, t_{n}^{k}\right] .
$$

In Proposition 3.3 we show that the approximate solutions introduced above indeed fulfill the discrete version of the energetic formulation from Definition 2.3. In order to check the discrete momentum equation (3.27b) and (3.27e), we shall make use of the following discrete by-part integration formula, for every $\left(r_{k}\right)_{k=1}^{n} \subset X$ and $\left(s_{k}\right)_{k=1}^{n} \subset X^{*}$, with $X$ a given Banach space:

$$
\sum_{k=1}^{n}\left\langle s_{k}, r_{k}-r_{k-1}\right\rangle_{X}=\left\langle s_{n}, r_{n}\right\rangle_{X}-\left\langle s_{0}, r_{0}\right\rangle_{X}-\sum_{k=1}^{n}\left\langle s_{k}-s_{k-1}, r_{k-1}\right\rangle_{X}
$$

In the discrete mechanical energy inequality (3.27c) below, the mechanical energy $\mathcal{E}$ will be replaced by

$\varepsilon_{n}(t, u, z):=\int_{\Omega}\left(\frac{1}{2} \mathbb{C}(z) e(u): e(u)+\frac{\tau_{n}}{\gamma}|e(u)|^{\gamma}\right) \mathrm{d} x+\mathcal{G}(z, \nabla z)-\left\langle\bar{f}_{n}(t), u\right\rangle_{H_{\mathrm{D}}^{1}\left(\Omega ; \mathbb{R}^{d}\right)}$ with $\tau_{n}=\frac{T}{n}$.

Proposition 3.3 (Time-discrete version of the energetic formulation (2.12) \& total energy inequality) Let the assumptions of Theorem 2.6 hold true. Then the interpolants of the timediscrete solutions $\left(\bar{u}_{n}, \underline{u}_{n}, u_{n}, \bar{z}_{n}, \underline{z}_{n}, z_{n}, \bar{\theta}_{n}, \underline{\theta}_{n}, \theta_{n}\right)$ obtained via Problem 3.1 and (3.23) satisfy the following properties:

- unidirectionality: for a.a. $x \in \Omega$, the functions $\bar{z}_{n}(\cdot, x):[0, T] \rightarrow[0,1]$ are nonincreasing;

- discrete semistability: for all $t \in[0, T]$

$$
\forall \tilde{z} \in Z: \quad \mathcal{E}_{n}\left(t, \underline{u}_{n}(t), \bar{z}_{n}(t)\right) \leq \mathcal{E}_{n}\left(t, \underline{u}_{n}(t), \tilde{z}\right)+\mathcal{R}_{1}\left(\tilde{z}-\bar{z}_{n}(t)\right)
$$

- discrete formulation of the momentum equation: for all $t \in[0, T]$ and for every $(n+$ 1)-tuple $\left(v_{n}^{k}\right)_{k=0, \ldots, n} \subset W_{\mathrm{D}}^{1, \gamma}\left(\Omega ; \mathbb{R}^{d}\right)$, setting $\bar{v}_{n}(s):=v_{n}^{k}$ and $v_{n}(s):=\frac{s-t_{n}^{k-1}}{\tau_{n}} v_{n}^{k}+$ $\frac{t_{n}^{k}-s}{\tau_{n}} v_{n}^{k-1}$ for $s \in\left(t_{n}^{k-1}, t_{n}^{k}\right]$, 


$$
\begin{aligned}
& \rho \int_{\Omega}\left(\dot{u}_{n}(t) \cdot \bar{v}_{n}(t)-\dot{u}_{0} \cdot v_{n}(0)\right) \mathrm{d} x-\rho \int_{0}^{\bar{\tau}_{n}(t)} \int_{\Omega} \dot{u}_{n}\left(s-\tau_{n}\right) \cdot \dot{v}_{n}(s) \mathrm{d} x \mathrm{~d} s \\
& \quad+\int_{0}^{\bar{\tau}_{n}(t)} \int_{\Omega}\left(\mathbb{D}\left(\underline{z}_{n}, \underline{\theta}_{n}\right) e\left(\dot{u}_{n}\right)+\mathbb{C}\left(\bar{z}_{n}\right) e\left(\bar{u}_{n}\right)-\bar{\theta}_{n} \mathbb{B}+\tau_{n}\left|e\left(\bar{u}_{n}\right)\right|^{\gamma-2} e\left(\bar{u}_{n}\right)\right): e\left(\bar{v}_{n}\right) \mathrm{d} x \mathrm{~d} s \\
& \quad=\int_{0}^{\bar{\tau}_{n}(t)}\left\langle\bar{f}_{n}, \bar{v}_{n}\right\rangle_{H_{\mathrm{D}}^{1}\left(\Omega ; \mathbb{R}^{d}\right)} \mathrm{d} s,
\end{aligned}
$$

where we have extended $u_{n}$ to $\left(-\tau_{n}, 0\right]$ by setting $u_{n}(t):=u_{n}^{0}+t \dot{u}_{0}$;

- discrete mechanical energy inequality: for all $t \in[0, T]$

$$
\begin{aligned}
& \frac{\rho}{2} \int_{\Omega}\left|\dot{u}_{n}(t)\right|^{2} \mathrm{~d} x+\varepsilon_{n}\left(t, \bar{u}_{n}(t), \bar{z}_{n}(t)\right)+\int_{\Omega}\left(z_{0}-\bar{z}_{n}(t)\right) \mathrm{d} x \\
& \quad+\int_{0}^{\bar{\tau}_{n}(t)} \int_{\Omega}\left(\mathbb{D}\left(\underline{z}_{n}, \underline{\theta}_{n}\right) e\left(\dot{u}_{n}\right)-\bar{\theta}_{n} \mathbb{B}\right): e\left(\dot{u}_{n}\right) \mathrm{d} x \mathrm{~d} s \\
& \quad \leq \frac{\rho}{2} \int_{\Omega}\left|\dot{u}_{0}\right|^{2} \mathrm{~d} x+\varepsilon_{n}\left(0, u_{n}^{0}, z_{0}\right)-\int_{0}^{\bar{\tau}_{n}(t)}\left\langle\dot{f}_{n}, \underline{u}_{n}\right\rangle_{H_{\mathrm{D}}^{1}\left(\Omega ; \mathbb{R}^{d}\right)} \mathrm{d} s ;
\end{aligned}
$$

- discrete total energy inequality: for all $t \in[0, T]$

$$
\begin{aligned}
& \frac{\rho}{2} \int_{\Omega}\left|\dot{u}_{n}(t)\right|^{2} \mathrm{~d} x+\varepsilon_{n}\left(t, \bar{u}_{n}(t), \bar{z}_{n}(t)\right)+\int_{\Omega} \bar{\theta}_{n}(t) \mathrm{d} x \\
& \quad \leq \frac{\rho}{2} \int_{\Omega}\left|\dot{u}_{0}\right|^{2} \mathrm{~d} x+\varepsilon_{n}\left(0, u_{n}^{0}, z_{0}\right)+\int_{\Omega} \theta_{0} \mathrm{~d} x \\
& \quad-\int_{0}^{\bar{\tau}_{n}(t)}\left\langle\dot{f}_{n}, \underline{u}_{n}\right\rangle_{H_{\mathrm{D}}^{1}\left(\Omega ; \mathbb{R}^{d}\right)} \mathrm{d} s+\int_{0}^{\bar{\tau}_{n}(t)}\left[\int_{\partial \Omega} \bar{h}_{n} \mathrm{~d} \mathcal{H}^{d-1}(x)+\int_{\Omega} \bar{H}_{n} \mathrm{~d} x\right] \mathrm{d} s
\end{aligned}
$$

- discrete formulation of the heat equation: for all $t \in[0, T]$ and for every $(n+1)$ tuple $\left(\eta_{n}^{k}\right)_{k=0}^{n} \subset H^{1}(\Omega)$, setting $\bar{\eta}_{n}(s):=\eta_{n}^{k}$ and $\eta_{n}(s):=\frac{s-t_{n}^{k-1}}{\tau_{n}} \eta_{n}^{k}+\frac{t_{n}^{k}-s}{\tau_{n}} \eta_{n}^{k-1}$ for $s \in\left(t_{n}^{k-1}, t_{n}^{k}\right]$,

$$
\begin{aligned}
& \int_{\Omega} \bar{\theta}_{n}(t) \bar{\eta}_{n}(t) \mathrm{d} x-\int_{\Omega} \theta_{0} \eta_{n}(0) \mathrm{d} x-\int_{0}^{\bar{\tau}_{n}(t)} \int_{\Omega} \underline{\theta}_{n}(s) \dot{\eta}_{n}(s) \mathrm{d} x \mathrm{~d} s \\
& \quad+\int_{0}^{\bar{\tau}_{n}(t)} \int_{\Omega}\left(\mathbb{K}\left(\bar{z}_{n}, \bar{\theta}_{n}\right) \nabla \bar{\theta}_{n}\right) \cdot \nabla \bar{\eta}_{n} \mathrm{~d} x \mathrm{~d} s \\
& =\int_{0}^{\bar{\tau}_{n}(t)} \int_{\Omega} \bar{\eta}_{n}\left|\dot{z}_{n}\right| \mathrm{d} x \mathrm{~d} s \int_{0}^{\bar{\tau}_{n}(t)} \int_{\Omega}\left(\mathbb{D}\left(\underline{z}_{n}, \underline{\theta}_{n}\right) e\left(\dot{u}_{n}\right)-\bar{\theta}_{n} \mathbb{B}\right): e\left(\dot{u}_{n}\right) \bar{\eta}_{n} \mathrm{~d} x \mathrm{~d} s \\
& \quad+\int_{0}^{\bar{\tau}_{n}(t)}\left[\int_{\partial \Omega} \bar{h}_{n} \eta_{n} \mathrm{~d} \mathcal{H}^{d-1}(x)+\left\langle\bar{H}_{n}, \eta_{n}\right\rangle_{H^{1}(\Omega)}\right] \mathrm{d} s .
\end{aligned}
$$

Proof The discrete momentum and heat equations (3.27b) and (3.27e) follow from testing (3.3b) and (3.3c) by the discrete test functions $\left(v_{n}^{k}\right)_{k=0}^{n} \subset W_{\mathrm{D}}^{1, \gamma}\left(\Omega ; \mathbb{R}^{d}\right)$ and $\left(\eta_{n}^{k}\right)_{k=0}^{n} \subset$ $H^{1}(\Omega)$, respectively, and applying the discrete by-part integration formula (3.25). From the discrete minimum problem (3.3a) we infer

$$
\begin{aligned}
& \mathcal{E}\left(t_{n}^{k}, u_{n}^{k-1}, z_{n}^{k}\right) \leq \mathcal{E}\left(t_{n}^{k}, u_{n}^{k-1}, \tilde{z}\right)+\int_{\Omega}\left(z_{n}^{k-1}-\tilde{z}\right) \mathrm{d} x \\
& \quad-\int_{\Omega}\left(z_{n}^{k-1}-z_{n}^{k}\right) \mathrm{d} x \leq \mathcal{E}\left(t_{n}^{k}, u_{n}^{k-1}, \tilde{z}\right)+\int_{\Omega}\left(z_{n}^{k}-\tilde{z}\right) \mathrm{d} x
\end{aligned}
$$


for all $\tilde{z} \in Z$ with $\tilde{z} \leq z_{n}^{k-1}$. By (3.3a) and the definition of the dissipation $\mathcal{R}_{1}$ we have $z_{n}^{k} \leq z_{n}^{k-1}$, whence the unidirectionality and the discrete semistability (3.27a) hold.

To deduce the mechanical energy inequality (3.27c) we choose $z_{n}^{k-1}$ as a competitor in (3.3a) and get

$$
\begin{aligned}
& \int_{\Omega}\left(z_{n}^{k-1}-z_{n}^{k}\right) \mathrm{d} x+\int_{\Omega}\left(\frac{1}{2} \mathbb{C}\left(z_{n}^{k}\right) e\left(u_{n}^{k-1}\right): e\left(u_{n}^{k-1}\right)+G\left(z_{n}^{k}, \nabla z_{n}^{k}\right)\right) \mathrm{d} x \\
& \quad \leq \int_{\Omega}\left(\frac{1}{2} \mathbb{C}\left(z_{n}^{k-1}\right) e\left(u_{n}^{k-1}\right): e\left(u_{n}^{k-1}\right)+G\left(z_{n}^{k-1}, \nabla z_{n}^{k-1}\right)\right) \mathrm{d} x .
\end{aligned}
$$

Moreover, we test (3.3b) by $v=u_{n}^{k}-u_{n}^{k-1}$. To this aim, we observe that by convexity (3.6)

$$
\begin{aligned}
& \rho \int_{\Omega} \frac{u_{n}^{k}-2 u_{n}^{k-1}+u_{n}^{k-2}}{\tau_{n}^{2}} \cdot\left(u_{n}^{k}-u_{n}^{k-1}\right) \mathrm{d} x \geq \rho \int_{\Omega}\left(\frac{1}{2} \frac{\left|u_{n}^{k}-u_{n}^{k-1}\right|^{2}}{\tau_{n}^{2}}-\frac{1}{2} \frac{\left|u_{n}^{k-1}-u_{n}^{k-2}\right|^{2}}{\tau_{n}^{2}}\right) \mathrm{d} x, \\
& \int_{\Omega} \mathbb{C}\left(z_{n}^{k}\right) e\left(u_{n}^{k}\right):\left(e\left(u_{n}^{k}\right)-e\left(u_{n}^{k-1}\right)\right) \mathrm{d} x \geq \int_{\Omega} \frac{1}{2}\left(\mathbb{C}\left(z_{n}^{k}\right) e\left(u_{n}^{k}\right): e\left(u_{n}^{k}\right)-\mathbb{C}\left(z_{n}^{k}\right) e\left(u_{n}^{k-1}\right): e\left(u_{n}^{k-1}\right)\right) \mathrm{d} x,
\end{aligned}
$$

$$
\int_{\Omega} \tau_{n}\left|e\left(u_{n}^{k}\right)\right|^{\gamma-2} e\left(u_{n}^{k}\right):\left(e\left(u_{n}^{k}\right)-e\left(u_{n}^{k-1}\right)\right) \mathrm{d} x \geq \int_{\Omega}\left(\frac{\tau_{n}}{\gamma}\left|e\left(u_{n}^{k}\right)\right|^{\gamma}-\frac{\tau_{n}}{\gamma}\left|e\left(u_{n}^{k-1}\right)\right|^{\gamma}\right) \mathrm{d} x .
$$

Further, let $t \in(0, T]$ be fixed, and let $1 \leq j \leq n$ fulfill $t \in\left(t_{n}^{j-1}, t_{n}^{j}\right]$. We sum (3.29a)(3.29c) over the index $k=1, \ldots, j$. Applying the by-part integration formula (3.25) we conclude that

$$
\begin{aligned}
& \sum_{k=1}^{j}\left\langle f_{n}^{k}, u_{n}^{k}-u_{n}^{k-1}\right\rangle_{H_{\mathrm{D}}^{1}\left(\Omega ; \mathbb{R}^{d}\right)}=\int_{0}^{\bar{\tau}_{n}(t)}\left\langle\bar{f}_{n}, \dot{u}_{n}\right\rangle_{H_{\mathrm{D}}^{1}\left(\Omega ; \mathbb{R}^{d}\right)} \mathrm{d} s \\
& \quad=\left\langle\bar{f}_{n}(t), \bar{u}_{n}(t)\right\rangle_{H_{\mathrm{D}}^{1}\left(\Omega ; \mathbb{R}^{d}\right)}-\left\langle f(0), u_{0}\right\rangle_{H_{\mathrm{D}}^{1}\left(\Omega ; \mathbb{R}^{d}\right)}-\int_{0}^{\bar{\tau}_{n}(t)}\left\langle\dot{f}_{n}, \underline{u}_{n}\right\rangle_{H_{\mathrm{D}}^{1}\left(\Omega ; \mathbb{R}^{d}\right)} \mathrm{d} s .
\end{aligned}
$$

All in all we infer

$$
\begin{aligned}
& \frac{\rho}{2} \int_{\Omega}\left|\dot{u}_{n}(t)\right|^{2} \mathrm{~d} x+\int_{0}^{\bar{\tau}_{n}(t)} \int_{\Omega}\left(\mathbb{D}\left(\underline{z}_{n}, \underline{\theta}_{n}\right) e\left(\dot{u}_{n}\right)-\underline{\theta}_{n} \mathbb{B}\right): e\left(\dot{u}_{n}\right) \mathrm{d} x \mathrm{~d} s \\
& \quad+\int_{\Omega} \frac{1}{2} \mathbb{C}\left(\bar{z}_{n}(t)\right) e\left(\bar{u}_{n}(t)\right): e\left(\bar{u}_{n}(t)\right) \mathrm{d} x+\int_{\Omega} \frac{\tau_{n}}{\gamma}\left|e\left(\bar{u}_{n}(t)\right)\right|^{\gamma} \mathrm{d} x-\left\langle\bar{f}_{n}(t), \bar{u}_{n}(t)\right\rangle_{H_{\mathrm{D}}^{1}\left(\Omega ; \mathbb{R}^{d}\right)} \\
& \quad \leq \frac{\rho}{2} \int_{\Omega}\left|\dot{u}_{0}\right|^{2} \mathrm{~d} x+\int_{\Omega} \frac{\tau_{n}}{\gamma}\left|e\left(u_{0}\right)\right|^{\gamma} \mathrm{d} x-\left\langle f(0), u_{0}\right\rangle_{H_{\mathrm{D}}^{1}\left(\Omega ; \mathbb{R}^{d}\right)}-\int_{0}^{\bar{\tau}_{n}(t)}\left\langle\dot{f}_{n}, \underline{u}_{n}\right\rangle_{H_{\mathrm{D}}^{1}\left(\Omega ; \mathbb{R}^{d}\right)} \mathrm{d} s \\
& \quad+\sum_{k=1}^{j} \int_{\Omega} \frac{1}{2} \mathbb{C}\left(z_{n}^{k}\right) e\left(u_{n}^{k-1}\right): e\left(u_{n}^{k-1}\right) \mathrm{d} x .
\end{aligned}
$$

We add the above inequality to (3.28), summed over $k=1, \ldots, j$. Observing the cancelation of the term $\sum_{k=1}^{j} \int_{\Omega} \frac{1}{2} \mathbb{C}\left(z_{n}^{k}\right) e\left(u_{n}^{k-1}\right): e\left(u_{n}^{k-1}\right) \mathrm{d} x$, we conclude (3.27c).

Finally, the discrete total energy inequality ensues from adding the discrete mechanical energy inequality (3.27c) with the discrete heat equation (3.3c), tested for $\eta=\tau_{n}$ and added up over $k=1, \ldots, j$. We observe the cancelation of some terms, and readily conclude (3.27d).

\section{它 Springer}




\subsection{A Priori Estimates}

The following result collects a series of a priori estimates on the approximate solutions, uniform with respect to $n \in \mathbb{N}$. Let us mention in advance that, in its proof we will start from the discrete total energy inequality (3.27d) and derive estimates (3.32a), (3.32b), (3.32d), (3.32h), for $\bar{u}_{n}, \dot{u}_{n}, \bar{z}_{n}$, as well as estimate (3.32i) below for $\left\|\bar{\theta}_{n}\right\|_{L^{\infty}\left(0, T ; L^{1}(\Omega)\right)}$. The next crucial step will be to obtain a bound for the $L^{2}\left(0, T ; H^{1}(\Omega)\right)$-norm of $\bar{\theta}_{n}$. For this, we will make use of a technique developed in [23], cf. also [63]. Namely, we will test the discrete heat equation (3.3c) by $\left(\theta_{n}^{k}\right)^{\alpha-1}$, with $\alpha \in(0,1)$. Exploiting the concavity of the function $F(\theta)=\theta^{\alpha} / \alpha$, we will deduce that

$$
\int_{0}^{T} \int_{\Omega} \mathbb{K}\left(\bar{z}_{n}, \bar{\theta}_{n}\right) \nabla\left(\bar{\theta}_{n}^{\alpha / 2}\right) \cdot \nabla\left(\bar{\theta}_{n}^{\alpha / 2}\right) \mathrm{d} x \mathrm{~d} t+\int_{\Omega} \frac{\theta_{0}^{\alpha}}{\alpha} \mathrm{d} x \leq \int_{\Omega} \frac{\bar{\theta}_{n}^{\alpha}(T)}{\alpha} \mathrm{d} x+C \int_{0}^{T} \int_{\Omega} \bar{\theta}_{n}^{\alpha+1}(t) \mathrm{d} x \mathrm{~d} t,
$$

where the positive and quadratic terms on the right-hand side of (3.3c) have been confined to the left-hand side and thus can be neglected. Hence, relying on the growth (2.6b) of $\mathbb{K}$, we will end up with an estimate for $\bar{\theta}_{n}^{\alpha / 2}$ in $L^{2}\left(0, T ; H^{1}(\Omega)\right)$, from which we will ultimately infer the desired bound $(3.32 \mathrm{j})$, whence $(3.32 \mathrm{k})$ by interpolation. We will be then in a position to exploit the mechanical energy inequality in order to recover the dissipative estimate (3.32c). Estimate (3.321) will finally ensue from a comparison in (3.3c).

In the following proof we will also use the concave counterpart to inequality (3.6), namely that for any concave (differentiable) function $\psi: \mathbb{R} \rightarrow(-\infty,+\infty]$

$$
\psi(x)-\psi(y) \leq \psi^{\prime}(y)(x-y) \text { for all } x, y \in \operatorname{dom}(\psi) .
$$

Proposition 3.4 (A priori estimates) Let the assumptions of Theorem 2.6 hold true and consider a sequence $\left(\bar{u}_{n}, \underline{u}_{n}, u_{n}, \bar{z}_{n}, \underline{z}_{n}, \bar{\theta}_{n}, \underline{\theta}_{n}, \theta_{n}\right)_{n}$ complying with Proposition 3.3 . Then there exists a constant $C>0$ such that the following estimates hold uniformly with respect to $n \in \mathbb{N}$ :

$$
\begin{aligned}
& \left\|\bar{u}_{n}\right\|_{L^{\infty}\left(0, T ; H_{\mathrm{D}}^{1}\left(\Omega ; \mathbb{R}^{d}\right)\right)} \leq C, \\
& \tau_{n}^{1 / \gamma}\left\|\bar{u}_{n}\right\|_{L^{\infty}\left(0, T ; W_{\mathrm{D}}^{1, \gamma}\left(\Omega ; \mathbb{R}^{d}\right)\right)} \leq C, \\
& \left\|u_{n}\right\|_{H^{1}\left(0, T ; H_{\mathrm{D}}^{1}\left(\Omega ; \mathbb{R}^{d}\right)\right)} \leq C, \\
& \left\|\dot{u}_{n}\right\|_{L^{\infty}\left(0, T ; L^{2}\left(\Omega ; \mathbb{R}^{d}\right)\right)} \leq C, \\
& \left\|\dot{u}_{n}\right\|_{\mathrm{BV}\left([0, T] ; W_{\mathrm{D}}^{1, \gamma}\left(\Omega ; \mathbb{R}^{d}\right)^{*}\right)} \leq C, \\
& \mathcal{R}_{1}\left(\bar{z}_{n}(T)-z_{0}\right) \leq C, \\
& \left\|\bar{z}_{n}\right\|_{L^{\infty}((0, T) \times \Omega)} \leq 1, \\
& \left\|\bar{z}_{n}\right\|_{L^{\infty}\left(0, T ; W^{1, q}(\Omega)\right)} \leq C, \\
& \left\|\bar{\theta}_{n}\right\|_{L^{\infty}\left(0, T ; L^{1}(\Omega)\right)} \leq C, \\
& \left\|\bar{\theta}_{n}\right\|_{L^{2}\left(0, T ; H^{1}(\Omega)\right)} \leq C, \\
& \left\|\bar{\theta}_{n}\right\|_{L^{p}((0, T) \times \Omega)} \leq C \text { for any } p \in\left\{\begin{array}{l}
{[1,8 / 3] \text { if } d=3,} \\
{[1,3] \text { if } d=2,}
\end{array}\right. \\
& \left\|\bar{\theta}_{n}\right\|_{\mathrm{BV}\left([0, T] ; W^{1, \infty}(\Omega)^{*}\right)} \leq C,
\end{aligned}
$$

where $\mathcal{R}_{1}$ is from (2.10). 
Observe that estimate (3.32c) implies (3.32a), and that (3.32k) is a consequence of (3.32i) and (3.32j). Nonetheless, we have chosen to highlight (3.32a) and (3.32k) for ease of exposition, both in the proof of Proposition 3.4 and for the compactness arguments of Proposition 4.1.

Proof Estimate (3.32f) follows from (2.5a), (2.7a), the definition of $\mathcal{R}_{1}$, and the monotonicity of $\bar{z}_{n}$ and $\underline{z}_{n}$. We divide the proof of the other estimates in subsequent steps.

First a priori estimates, ad (3.32a), (3.32b), (3.32d), (3.32g), (3.32h), (3.32i): We start from the discrete total energy inequality $(3.27 \mathrm{~d})$. For its left-hand side, we observe that the first and the third term are nonnegative. For the second one, we use that, in view of (2.3d), (2.5d), and (2.8a), we have

$$
\begin{aligned}
\mathcal{E}_{n}\left(t, \bar{u}_{n}(t), \bar{z}_{n}(t)\right) \geq & C_{\mathbb{C}}^{1} \int_{\Omega}\left|e\left(\bar{u}_{n}(t)\right)\right|^{2} \mathrm{~d} x+C_{G}^{1} \int_{\Omega}\left|\nabla \bar{z}_{n}(t)\right|^{q} \mathrm{~d} x+\frac{\tau_{n}}{\gamma} \int_{\Omega}\left|e\left(\bar{u}_{n}(t)\right)\right|^{\gamma} \mathrm{d} x \\
& -\left\|\bar{f}_{n}\right\|_{L^{\infty}\left(0, T ; H_{\mathrm{D}}^{1}\left(\Omega ; \mathbb{R}^{d}\right)^{*}\right)}\left\|\bar{u}_{n}(t)\right\|_{H_{\mathrm{D}}^{1}\left(\Omega ; \mathbb{R}^{d}\right)}-C \\
\geq & C\left(\left\|\bar{u}_{n}(t)\right\|_{H_{\mathrm{D}}^{1}\left(\Omega ; \mathbb{R}^{d}\right)}^{2}+\tau_{n}\left\|\bar{u}_{n}(t)\right\|_{W_{\mathrm{D}}^{1, \gamma}\left(\Omega ; \mathbb{R}^{d}\right)}^{\gamma}+\left\|\bar{z}_{n}(t)\right\|_{W^{1, q}(\Omega)}^{q}\right)-C,
\end{aligned}
$$

for almost all $t \in(0, T)$, where we have also used Poincaré's and Korn's inequalities. Concerning the right-hand side of $(3.27 \mathrm{~d})$, we use that $\left|\partial_{t} \varepsilon_{n}\left(t, \underline{u}_{n}(t), \underline{z}_{n}(t)\right)\right| \leq$ $\left\|\dot{f}_{n}\right\|_{H_{\mathrm{D}}^{1}\left(\Omega ; \mathbb{R}^{d}\right)^{*}}\left\|\underline{u}_{n}(t)\right\|_{H_{\mathrm{D}}^{1}\left(\Omega ; \mathbb{R}^{d}\right)}$ for almost all $t \in(0, T)$. The remaining terms on the righthand side are bounded, uniformly with respect to $n \in \mathbb{N}$, in view of the properties of the initial and given data (2.7) and (3.2), and of (3.24d). All in all, from (3.27d) we deduce

$$
C\left\|\bar{u}_{n}(t)\right\|_{H_{\mathrm{D}}^{1}\left(\Omega ; \mathbb{R}^{d}\right)}^{2} \leq C+\frac{1}{2} \int_{0}^{\bar{\tau}_{n}(t)}\left\|\underline{u}_{n}(s)\right\|_{H_{\mathrm{D}}^{1}\left(\Omega ; \mathbb{R}^{d}\right)}^{2} \mathrm{~d} s+\frac{1}{2} \int_{0}^{\bar{\tau}_{n}(t)}\left\|\dot{f}_{n}\right\|_{H_{\mathrm{D}}^{1}\left(\Omega ; \mathbb{R}^{d}\right)^{*}}^{2} \mathrm{~d} s .
$$

Also in view of the bounds on $\dot{f}_{n}$ by (3.24c), estimate (3.32a) then follows from the Gronwall Lemma. As a by-product, we conclude that

$$
\int_{0}^{\bar{\tau}_{n}(t)}\left|\partial_{t} \mathcal{E}_{n}\left(s, \underline{u}_{n}(s), \underline{z}_{n}(s)\right)\right| \mathrm{d} s \leq C \int_{0}^{\bar{\tau}_{n}(t)}\left\|\dot{f}_{n}(s)\right\|_{H_{\mathrm{D}}^{1}\left(\Omega ; \mathbb{R}^{d}\right)^{*}} \mathrm{~d} s \leq C .
$$

Inserting this into $(3.27 \mathrm{~d})$ we also infer estimates (3.32d), (3.32i), and that $\left|\mathcal{E}_{n}\left(t, \bar{u}_{n}(t), \bar{z}_{n}(t)\right)\right| \leq C$ for a constant independent of $n \in \mathbb{N}$ and $t \in(0, T)$. This implies (3.32b) and the first estimate in (3.32h) via (3.33). Then the second estimate in $(3.32 \mathrm{~h})$ immediately follows from the very definition of the interpolants (3.23). Moreover, $(3.32 \mathrm{~g})$ is a direct consequence of the boundedness of the energy, which implies $\bar{z}_{n}, \underline{z}_{n} \in[0,1]$ a.e. in $\Omega$, for a.e. $t \in(0, T)$.

Second a priori estimate: We fix $\alpha \in(0,1)$. Exploiting that $\theta_{n}^{k} \geq \widetilde{\theta}>0$, we may test (3.3c) by $\left(\theta_{n}^{k}\right)^{\alpha-1}$, thus obtaining

$$
\begin{aligned}
& \frac{4(1-\alpha)}{\alpha^{2}} \int_{\Omega} \mathbb{K}\left(z_{n}^{k}, \theta_{n}^{k}\right) \nabla\left(\theta_{n}^{k}\right)^{\alpha / 2} \cdot \nabla\left(\theta_{n}^{k}\right)^{\alpha / 2} \mathrm{~d} x+\int_{\Omega} \mathbb{D}\left(z_{n}^{k}\right) e\left(\frac{u_{n}^{k}-u_{n}^{k-1}}{\tau}\right): e\left(\frac{u_{n}^{k}-u_{n}^{k-1}}{\tau}\right)\left(\theta_{n}^{k}\right)^{\alpha-1} \mathrm{~d} x \\
& \quad+\int_{\Omega} \frac{z_{n}^{k-1}-z_{n}^{k}}{\tau}\left(\theta_{n}^{k}\right)^{\alpha-1} \mathrm{~d} x+\left\langle H_{n}^{k},\left(\theta_{n}^{k}\right)^{\alpha-1}\right\rangle_{H^{1}(\Omega)}+\int_{\partial \Omega} h_{n}^{k}\left(\theta_{n}^{k}\right)^{\alpha-1} \mathrm{~d} \mathcal{H}^{d-1} \\
& =\int_{\Omega} \frac{\theta_{n}^{k}-\theta_{n}^{k-1}}{\tau}\left(\theta_{n}^{k}\right)^{\alpha-1} \mathrm{~d} x+\int_{\Omega} \theta_{n}^{k} \mathbb{B}: e\left(\frac{u_{n}^{k}-u_{n}^{k-1}}{\tau}\right)\left(\theta_{n}^{k}\right)^{\alpha-1} \mathrm{~d} x \doteq I_{1}+I_{2},
\end{aligned}
$$


where we used that

$$
\begin{aligned}
\mathbb{K}\left(z_{n}^{k}, \theta_{n}^{k}\right) \nabla \theta_{n}^{k} \cdot \nabla\left(\theta_{n}^{k}\right)^{\alpha-1} & =(\alpha-1)\left(\theta_{n}^{k}\right)^{\alpha-2} \mathbb{K}\left(z_{n}^{k}, \theta_{n}^{k}\right) \nabla \theta_{n}^{k} \cdot \nabla \theta_{n}^{k} \\
& =\frac{4(\alpha-1)}{\alpha^{2}} \mathbb{K}\left(z_{n}^{k}, \theta_{n}^{k}\right) \nabla\left(\theta_{n}^{k}\right)^{\alpha / 2} \cdot \nabla\left(\theta_{n}^{k}\right)^{\alpha / 2}
\end{aligned}
$$

and moved the term $\int_{\Omega} \mathbb{K}\left(z_{n}^{k}, \theta_{n}^{k}\right) \nabla \theta_{n}^{k} \nabla\left(\theta_{n}^{k}\right)^{\alpha-1} \mathrm{~d} x$ to the opposite side. It follows from (3.31) with $\psi(x):=\frac{x^{\alpha}}{\alpha}$ that

$$
I_{1} \leq \int_{\Omega} \psi\left(\theta_{n}^{k}\right) \mathrm{d} x-\int_{\Omega} \psi\left(\theta_{n}^{k-1}\right) \mathrm{d} x,
$$

whereas we estimate $I_{2}$ by

$$
I_{2} \leq \frac{C_{\mathbb{D}}^{1}}{2} \int_{\Omega}\left|e\left(\frac{u_{n}^{k}-u_{n}^{k-1}}{\tau}\right)\right|^{2}\left(\theta_{n}^{k}\right)^{\alpha-1} \mathrm{~d} x+C \int_{\Omega}\left|\theta_{n}^{k}\right|^{2}\left(\theta_{n}^{k}\right)^{\alpha-1} \mathrm{~d} x \doteq I_{3}+I_{4},
$$

where $C_{\mathbb{D}}^{1}$ from (2.3e) is such that $\int_{\Omega} \mathbb{D}\left(z_{n}^{k}\right) e\left(\frac{u_{n}^{k}-u_{n}^{k-1}}{\tau}\right): e\left(\frac{u_{n}^{k}-u_{n}^{k-1}}{\tau}\right)\left(\theta_{n}^{k}\right)^{\alpha-1} \mathrm{~d} x$ on the lefthand side of (3.35) is bounded from below by $C_{\mathbb{D}}^{1} \int_{\Omega}\left|e\left(\frac{u_{n}^{k}-u_{n}^{k-1}}{\tau}\right)\right|^{2}\left(\theta_{n}^{k}\right)^{\alpha-1} \mathrm{~d} x$, which in turn dominates $I_{3}$. Taking into account that the second, the third and the fourth integrals on the left-hand side of (3.35) are nonnegative also thanks to (2.8b) and summing up over the index $k$, we end up with

$$
\begin{aligned}
& \frac{4(1-\alpha)}{\alpha^{2}} \int_{0}^{\bar{\tau}_{n}(t)} \int_{\Omega} \mathbb{K}\left(\bar{z}_{n}, \bar{\theta}_{n}\right) \nabla\left(\bar{\theta}_{n}^{\alpha / 2}\right) \cdot \nabla\left(\bar{\theta}_{n}^{\alpha / 2}\right) \mathrm{d} x \mathrm{~d} s+\int_{\Omega} \frac{\theta_{0}^{\alpha}}{\alpha} \mathrm{d} x \\
& \leq \int_{\Omega} \frac{\bar{\theta}_{n}(t)^{\alpha}}{\alpha} \mathrm{d} x+C \int_{0}^{\bar{\tau}_{n}(t)} \int_{\Omega} \bar{\theta}_{n}(t)^{\alpha+1} \mathrm{~d} x \mathrm{~d} s .
\end{aligned}
$$

Since $\alpha \in(0,1)$ and $\theta_{n}^{k} \geq \widetilde{\theta}>0$, we have

$$
\int_{\Omega} \frac{\bar{\theta}_{n}(t)^{\alpha}}{\alpha} \mathrm{d} x \leq \frac{1}{\alpha} \int_{\Omega} \bar{\theta}_{n}(t) \mathrm{d} x+C \leq C,
$$

where the latter estimate follows by (3.32i). From (2.6b) we deduce that

$$
\begin{gathered}
\int_{0}^{\bar{\tau}_{n}(t)} \int_{\Omega} \mathbb{K}\left(\bar{z}_{n}, \bar{\theta}_{n}\right) \nabla\left(\bar{\theta}_{n}^{\alpha / 2}\right) \cdot \nabla\left(\bar{\theta}_{n}^{\alpha / 2}\right) \mathrm{d} x \mathrm{~d} s \geq c_{1} \int_{0}^{\bar{\tau}_{n}(t)} \int_{\Omega}\left(\bar{\theta}_{n}\right)^{\kappa}\left|\nabla\left(\bar{\theta}_{n}^{\alpha / 2}\right)\right|^{2} \mathrm{~d} x \mathrm{~d} s \\
=C \int_{0}^{\bar{\tau}_{n}(t)} \int_{\Omega}\left|\left(\bar{\theta}_{n}\right)^{\kappa+\alpha-2}\right|\left|\nabla \bar{\theta}_{n}\right|^{2} \mathrm{~d} x \mathrm{~d} s=C \int_{0}^{\bar{\tau}_{n}(t)} \int_{\Omega}\left|\nabla\left(\bar{\theta}_{n}^{(\kappa+\alpha) / 2}\right)\right|^{2} \mathrm{~d} x \mathrm{~d} s .
\end{gathered}
$$

In order to clarify the estimate for the second term on the right-hand side of (3.36), we now use the placeholder

$$
w_{n}:=\left(\bar{\theta}_{n}\right)^{(\kappa+\alpha) / 2},
$$

so that $\left(\bar{\theta}_{n}\right)^{\alpha+1}=\left(w_{n}\right)^{2(\alpha+1) /(\alpha+\kappa)}$. Hence, neglecting the (positive) second term on the left-hand side of (3.36), we infer

$$
\int_{0}^{\bar{\tau}_{n}(t)} \int_{\Omega}\left|\nabla w_{n}\right|^{2} \mathrm{~d} x \mathrm{~d} s \leq C+C \int_{0}^{\bar{\tau}_{n}(t)} \int_{\Omega}\left|w_{n}\right|^{\omega} \mathrm{d} x \mathrm{~d} s \quad \text { with } \omega=2 \frac{\alpha+1}{\alpha+\kappa} .
$$

We now proceed exactly in the same way as in [23], cf. also [63]. Namely, the GagliardoNirenberg inequality for $d=3$ (for $d=2$ even better estimates hold true) yields

$$
\left\|w_{n}\right\|_{L^{\omega}(\Omega)} \leq C\left\|\nabla w_{n}\right\|_{L^{2}\left(\Omega ; \mathbb{R}^{d}\right)}^{\sigma}\left\|w_{n}\right\|_{L^{r}(\Omega)}^{1-\sigma}+C^{\prime}\left\|w_{n}\right\|_{L^{r}(\Omega)}
$$


for suitable constants $C$ and $C^{\prime}$, and for $1 \leq r \leq \omega$ and $\sigma$ satisfying $1 / \omega=\sigma / 6+(1-\sigma) / r$. Hence $\sigma=6(\omega-r) / \omega(6-r)$. Observe that $\sigma \in(0,1)$ since $\omega=2(\alpha+1) /(\alpha+\kappa)<6$, which is satisfied because $\kappa>1$. Hence we transfer the Gagliardo-Nirenberg estimate into (3.38) and use Young's inequality in the estimate of the term

$$
\begin{aligned}
& C \int_{0}^{\bar{\tau}_{n}(t)}\left\|\nabla w_{n}\right\|_{L^{2}\left(\Omega ; \mathbb{R}^{d}\right)}^{\omega \sigma}\left\|w_{n}\right\|_{L^{r}(\Omega)}^{\omega(1-\sigma)} \mathrm{d} s \\
& \leq \frac{1}{2} \int_{0}^{\bar{\tau}_{n}(t)}\left\|\nabla w_{n}\right\|_{L^{2}\left(\Omega ; \mathbb{R}^{d}\right)}^{2} \mathrm{~d} s \\
& \quad+C^{\prime} \int_{0}^{\bar{\tau}_{n}(t)}\left\|w_{n}\right\|_{L^{r}(\Omega)}^{2 \omega(1-\sigma) /(2-\omega \sigma)} \mathrm{d} s .
\end{aligned}
$$

In the previous inequality we have used the fact that $\omega \sigma<2$, which holds since $\omega<2$ and $\sigma<1$ by (3.38). The term $\frac{1}{2} \int_{0}^{\bar{\tau}_{n}(t)}\left\|\nabla w_{n}\right\|_{L^{2}\left(\Omega ; \mathbb{R}^{d}\right)}^{2} \mathrm{~d} s$ may be absorbed into the left-hand side of (3.38). All in all, we conclude

$$
\int_{0}^{\bar{\tau}_{n}(t)} \int_{\Omega}\left|\nabla w_{n}\right|^{2} \mathrm{~d} x \mathrm{~d} s \leq C+C \int_{0}^{\bar{\tau}_{n}(t)}\left\|w_{n}\right\|_{L^{r}(\Omega)}^{2 \omega(1-\sigma) /(2-\omega \sigma)} \mathrm{d} s+C^{\prime} \int_{0}^{\bar{\tau}_{n}(t)}\left\|w_{n}\right\|_{L^{r}(\Omega)}^{\omega} \mathrm{d} s .
$$

Now, let us choose

$$
1 \leq r \leq 2 /(\alpha+\kappa) .
$$

Then, we have for almost all $t \in(0, T)$ that

$$
\left\|w_{n}(t)\right\|_{L^{r}(\Omega)}=\left(\int_{\Omega}\left(\bar{\theta}_{n}(t)\right)^{r(\kappa+\alpha) / 2} \mathrm{~d} x\right)^{1 / r}=\left(\int_{\Omega} \bar{\theta}_{n}(t) \mathrm{d} x\right)^{1 / r} \leq C
$$

for a constant independent of $t$, where again we have used estimate (3.32i). Observe that, since we have previously imposed $\kappa+\alpha-2 \geq 0$, we ultimately find that (3.40) must hold for $r=1$ and that, moreover, $\alpha=2-\kappa \in\left(2-\kappa_{d}, 1\right)$, with $\kappa_{d}=5 / 3$ if $d=3$ and $\kappa_{d}=2$ if $d=2$, so that $w_{n}=\bar{\theta}_{n}$. From (3.39)-(3.40) we then infer

$$
\int_{0}^{\bar{\tau}_{n}(t)} \int_{\Omega}\left|\nabla \bar{\theta}_{n}\right|^{2} \mathrm{~d} x \mathrm{~d} s \leq C .
$$

Third a priori estimate, ad (3.32j) and (3.32k): From (3.41) we deduce (3.32j) in view of the previously obtained (3.32i) via Poincaré's inequality. Estimate $(3.32 \mathrm{k})$ ensues by interpolation between $L^{2}\left(0, T ; H^{1}(\Omega)\right)$ and $L^{\infty}\left(0, T ; L^{1}(\Omega)\right)$, relying on (3.32j) and (3.32i) and exploiting the Gagliardo-Nirenberg inequality. For later convenience, let us also point out that, we indeed recover the following bound

$$
\left\|\left(\bar{\theta}_{n}\right)^{(\kappa+\alpha) / 2}\right\|_{L^{2}\left(0, T ; H^{1}(\Omega)\right)} \leq C
$$

for arbitrary $\alpha \in(0,1)$. For this, it is sufficient to observe that second term on the right-hand side of (3.36) now fulfills $\int_{0}^{\bar{\tau}_{n}(t)} \int_{\Omega} \bar{\theta}_{n}(t)^{\alpha+1} \mathrm{~d} x \mathrm{~d} s \leq C$ thanks to estimate (3.32k). Then, by (3.37) we find that $\int_{0}^{\bar{\tau}_{n}(t)} \int_{\Omega}\left|\nabla\left(\bar{\theta}_{n}^{(\kappa+\alpha) / 2}\right)\right|^{2} \mathrm{~d} x \mathrm{~d} s \leq C$, whence (3.42) via Poincaré's inequality.

Fourth a priori estimate, ad (3.32c) and (3.32e): From the discrete mechanical energy inequality $(3.27 \mathrm{c})$ we infer

$$
C_{\mathbb{D}}^{1} \int_{0}^{\bar{\tau}_{n}(t)} \int_{\Omega}\left|e\left(\dot{u}_{n}\right)\right|^{2} \mathrm{~d} x \mathrm{~d} s \leq C+\int_{0}^{\bar{\tau}_{n}(t)} \int_{\Omega} \bar{\theta}_{n} \mathbb{B}: e\left(\dot{u}_{n}\right) \mathrm{d} x \mathrm{~d} s
$$

\section{Springer}


where we have used (3.33), (3.34), and the fact that the terms $\int_{\Omega}\left|\dot{u}_{0}\right|^{2} \mathrm{~d} x$ and $\mathcal{E}\left(0, u_{n}^{0}, z_{0}\right)$ are bounded, uniformly with respect to $n \in \mathbb{N}$, in view of (2.7a) and (3.2). Exploiting the previously obtained estimate $(3.32 \mathrm{j})$ we find

$$
\begin{aligned}
\int_{0}^{\bar{\tau}_{n}(t)} \int_{\Omega} \bar{\theta}_{n} \mathbb{B}: e\left(\dot{u}_{n}\right) \mathrm{d} x \mathrm{~d} t & \leq \frac{C_{\mathbb{D}}^{1}}{2} \int_{0}^{\bar{\tau}_{n}(t)} \int_{\Omega}\left|e\left(\dot{u}_{n}\right)\right|^{2} \mathrm{~d} x \mathrm{~d} t+C \int_{0}^{\bar{\tau}_{n}(t)} \int_{\Omega}\left|\bar{\theta}_{n}\right|^{2} \mathrm{~d} x \mathrm{~d} s \\
& \leq \frac{C_{\mathbb{D}}^{1}}{2} \int_{0}^{\bar{\tau}_{n}(t)} \int_{\Omega}\left|e\left(\dot{u}_{n}\right)\right|^{2} \mathrm{~d} x \mathrm{~d} t+C .
\end{aligned}
$$

Inserting this into (3.43) we conclude (3.32c) via Korn's inequality, again exploiting the definition of the interpolants (3.23). Finally, estimate (3.32e) ensues from a comparison argument in (3.3b), taking into account the previously proven $(3.32 \mathrm{~b}),(3.32 \mathrm{c}),(3.32 \mathrm{j})$, as well as (3.24a).

Fifth a priori estimate, ad (3.321): Let $\kappa$ be as in (2.6). In (3.3c) we use a test function $\eta \in W^{1, \infty}(\Omega)$, thus we find

$$
\left|\int_{\Omega} \frac{\theta_{n}^{k}-\theta_{n}^{k-1}}{\tau_{n}} \eta \mathrm{d} x\right| \leq\left|\int_{\Omega} \mathbb{K}\left(z_{n}^{k}, \theta_{n}^{k}\right) \nabla \theta_{n}^{k} \cdot \nabla \eta \mathrm{d} x\right|+\left|\left\langle\operatorname{RHS}_{n}^{k}, \eta\right\rangle_{W^{1, \infty}(\Omega)}\right|,
$$

where the terms on the right-hand side of (3.3c) are summarized in $\mathrm{RHS}_{n}^{k}$. It follows from assumptions (2.3) and (2.8b) that

$$
\begin{aligned}
& \left|\left\langle\operatorname{RHS}_{n}^{k}, \eta\right\rangle_{W^{1, \infty}(\Omega)}\right| \\
& \leq C\left(\left\|e\left(\frac{u_{n}^{k}-u_{n}^{k-1}}{\tau_{n}}\right)\right\|_{L^{2}\left(\Omega ; \mathbb{R}^{d \times d}\right)}^{2}+\left\|\theta_{n}^{k}\right\|_{L^{2}(\Omega)}^{2}+\left\|\frac{z_{n}^{k}-z_{n}^{k-1}}{\tau_{n}}\right\|_{L^{1}(\Omega)}\right. \\
& \left.\quad+\left\|h_{n}^{k}\right\|_{L^{2}(\partial \Omega)}+\left\|H_{n}^{k}\right\|_{L^{1}(\Omega)}\right)\|\eta\|_{L^{\infty}(\Omega)} \\
& \doteq \Lambda_{n}^{k}\|\eta\|_{L^{\infty}(\Omega)} .
\end{aligned}
$$

Furthermore, with (2.6) we find for every $\alpha \in(1 / 2,1)$

$$
\begin{aligned}
& \left|\int_{\Omega} \mathbb{K}\left(z_{n}^{k}, \theta_{n}^{k}\right) \nabla \theta_{n}^{k} \cdot \nabla \eta \mathrm{d} x\right| \\
& \leq\|\nabla \eta\|_{L^{\infty}\left(\Omega ; \mathbb{R}^{d}\right)} c_{2}\left\|\left(\left(\theta_{n}^{k}\right)^{\kappa}+1\right) \nabla \theta_{n}^{k}\right\|_{L^{1}\left(\Omega ; \mathbb{R}^{d}\right)} \\
& \leq\|\nabla \eta\|_{L^{\infty}\left(\Omega ; \mathbb{R}^{d}\right)} c_{2}\left(\left\|\left(\theta_{n}^{k}\right)^{(\kappa-\alpha+2) / 2}\right\|_{L^{2}(\Omega)}\left\|\left(\theta_{n}^{k}\right)^{(\kappa+\alpha-2) / 2} \nabla \theta_{n}^{k}\right\|_{L^{2}\left(\Omega ; \mathbb{R}^{d}\right)}\right. \\
& \left.+\mathcal{L}^{d}(\Omega)^{1 / 2}\left\|\nabla \theta_{n}^{k}\right\|_{L^{2}\left(\Omega ; \mathbb{R}^{d}\right)}\right) .
\end{aligned}
$$

Inserting (3.45) and (3.46) into (3.44) and summing over the index $k=1, \ldots, n$, we find for every time-dependent function $\eta \in \mathrm{C}^{0}\left([0, T] ; W^{1, \infty}(\Omega)\right)$ that

$$
\begin{aligned}
& \mid \begin{array}{l}
\left|\int_{0}^{\bar{\tau}_{n}(t)} \int_{\Omega} \dot{\theta}_{n} \eta \mathrm{d} x \mathrm{~d} s\right| \\
\leq C\|\nabla \eta\|_{L^{\infty}\left((0, T) \times \Omega ; \mathbb{R}^{d}\right)}\left(\left\|\bar{\theta}_{n}\right\|_{L^{\kappa-\alpha+2}((0, T) \times \Omega)}^{(\kappa-\alpha+2) / 2}\left\|\left(\bar{\theta}_{n}\right)^{(\kappa+\alpha) / 2}\right\|_{L^{2}\left(0, T ; H^{1}(\Omega)\right)}\right. \\
\left.+\left\|\nabla \bar{\theta}_{n}\right\|_{L^{2}\left((0, T) \times \Omega ; \mathbb{R}^{d}\right)}\right)
\end{array} \\
& \quad+\|\eta\|_{L^{\infty}((0, T) \times \Omega)} \int_{0}^{\bar{\tau}_{n}(t)} \bar{\Lambda}_{n} \mathrm{~d} s
\end{aligned}
$$


where $\bar{\Lambda}_{n}$ denotes the piecewise constant interpolant of the values $\left(\Lambda_{n}^{k}\right)_{k}$. Note that the estimate on $\left\|\left(\theta_{n}^{k}\right)^{(\kappa+\alpha-2) / 2} \nabla \theta_{n}^{k}\right\|_{L^{2}\left(\Omega ; \mathbb{R}^{d}\right)}$ ensues from (3.37) and (3.42). Now, observe that

$$
\left\|\bar{\theta}_{n}\right\|_{L^{\kappa-\alpha+2}((0, T) \times \Omega)}^{(\kappa-\alpha+2) / 2} \leq C
$$

thanks to (3.32k) if $p=\kappa-\alpha+2$ satisfies the constraints in (3.32k). Recall that the parameter $\alpha$ for which (3.42) holds can be chosen arbitrarily close to 1 . Therefore, such constraints for $p=\kappa-\alpha+2$ are valid since, by (2.6b), $\kappa \in\left(1, \kappa_{d}\right)$ with $\kappa_{d}=5 / 3$ if $d=3$ and $\kappa_{d}=2$ if $d=2$. Finally, it follows from (3.24d), (3.32c), (3.32f), and (3.32j) that $\int_{0}^{T} \bar{\Lambda}_{n} \mathrm{~d} t \leq C$. Ultimately, from (3.47) we conclude (3.321).

\section{Passage from Time-Discrete to Continuous}

Based on the a priori bounds deduced in Proposition 3.4, exploiting compactness results à la Aubin-Lions as well as a version of Helly's selection principle, we are now in a position to extract a subsequence of solutions of the time-discrete problems converging to a limit triple $(u, z, \theta)$ in suitable topologies. In (4.1) below we have collected all of these convergences with some redundancies: for example, (4.1g) and (4.1i) imply (4.1h) and (4.1j), but the latter are stated for later reference. Subsequently, we will verify that the triple $(u, z, \theta)$ is an energetic solution of the time-continuous problem as stated in Definition 2.3.

Proposition 4.1 (Convergence of the time-discrete solutions) Let the assumptions of Theorem 2.6 be satisfied. Then, there exists a triple $(u, z, \theta):[0, T] \times \Omega \rightarrow \mathbb{R}^{d} \times \mathbb{R} \times[0, \infty)$ of regularity (2.11) such that for a.a. $x \in \Omega$ the function $t \mapsto z(t, x) \in[0,1]$ is nonincreasing, (2.14) holds, as well as (2.16) under the assumption (2.15), and there exists a subsequence of the time-discrete solutions $\left(\bar{u}_{n}, \underline{u}_{n}, u_{n}, \bar{z}_{n}, \underline{z}_{n}, \bar{\theta}_{n}, \underline{\theta}_{n}\right)_{n}$ from (3.23) such that

\begin{tabular}{|c|c|c|}
\hline $\bar{u}_{n} \stackrel{*}{\rightarrow} u$ & in $L^{\infty}\left(0, T ; H_{\mathrm{D}}^{1}\left(\Omega ; \mathbb{R}^{d}\right)\right)$ & (4.1a) \\
\hline$u_{n} \rightarrow u$ & in $H^{1}\left(0, T ; H_{\mathrm{D}}^{1}\left(\Omega ; \mathbb{R}^{d}\right)\right)$, & $(4.1 b)$ \\
\hline$\dot{u}_{n} \stackrel{*}{\rightarrow} \dot{u}$ & in $L^{\infty}\left(0, T ; L^{2}\left(\Omega ; \mathbb{R}^{d}\right)\right)$, & $(4.1 c)$ \\
\hline $\bar{u}_{n}(t), u_{n}(t) \rightarrow u(t)$ & in $H_{\mathrm{D}}^{1}\left(\Omega ; \mathbb{R}^{d}\right)$ for all $t \in[0, T]$ & $(4.1 d)$ \\
\hline$\dot{u}_{n}(t) \rightarrow \dot{u}(t)$ & in $L^{2}\left(\Omega ; \mathbb{R}^{d}\right)$ for all $t \in[0, T]$ & $(4.1 \mathrm{e})$ \\
\hline $\bar{z}_{n}, \underline{z}_{n} \stackrel{*}{\rightarrow} z$ & in $L^{\infty}\left(0, T ; W^{1, q}(\Omega)\right) \cap L^{\infty}((0, T) \times \Omega)$ & $(4.1 f)$ \\
\hline $\bar{z}_{n}(t) \rightarrow z(t)$ & in $W^{1, q}(\Omega)$ for all $t \in[0, T]$ & $(4.1 \mathrm{~g})$ \\
\hline $\bar{z}_{n}(t) \rightarrow z(t)$ & in $L^{r}(\Omega)$ for all $r \in[1, \infty)$ and for all $t \in[0, T]$, & $(4.1 h)$ \\
\hline$\underline{z}_{n}(t) \rightarrow z(t)$ & in $W^{1, q}(\Omega)$ for all $t \in[0, T] \backslash J$ & (4.1i) \\
\hline$\underline{z}_{n}(t) \rightarrow z(t)$ & in $L^{r}(\Omega)$ for all $r \in[1, \infty)$ and for all $t \in[0, T] \backslash J$, & $(4.1 \mathrm{j})$ \\
\hline $\bar{\theta}_{n}, \underline{\theta}_{n} \rightarrow \theta$ & in $L^{2}\left(0, T ; H^{1}(\Omega)\right)$ & $(4.1 \mathrm{k})$ \\
\hline $\bar{\theta}_{n}, \underline{\theta}_{n}, \theta_{n} \rightarrow \theta$ & in $L^{2}(0, T ; Y)$ for all $Y$ such that $H^{1}(\Omega) \Subset Y \subset W$ & $\begin{array}{r}\delta(\Omega)^{*} \\
(4.11)\end{array}$ \\
\hline $\bar{\theta}_{n}, \underline{\theta}_{n}, \theta_{n} \rightarrow \theta$ & in $L^{p}((0, T) \times \Omega)$ for all $p \in \begin{cases}{[1,8 / 3)} & \text { if } d=3, \\
{[1,3)} & \text { if } d=2,\end{cases}$ & $(4.1 \mathrm{~m})$ \\
\hline$\theta_{n}(t) \rightarrow \theta(t)$ & in $W^{2, d+\delta}(\Omega)^{*}$ for all $t \in[0, T]$, & $(4.1 n)$ \\
\hline
\end{tabular}


The set $J \subset[0, T]$ appearing in (4.1i)-(4.1j) denotes the jump set of $z \in \mathrm{BV}\left([0, T] ; L^{1}(\Omega)\right)$. Finally,

$$
\left|\dot{z}_{n}\right| \rightarrow|\dot{z}| \text { in the sense of measures on }[0, T] \times \bar{\Omega} \text {. }
$$

Proof Convergence of the displacements: The convergences (4.1a), (4.1b), and (4.1c) follow by compactness from (3.32a), (3.32c), and (3.32d). As $u_{n}(t)-\bar{u}_{n}(t)=\left(t-t_{n}^{k}\right) \dot{u}_{n}(t)$ and $u_{n}(t)-\underline{u}_{n}(t)=\left(t-t_{n}^{k-1}\right) \dot{u}_{n}(t)$, we immediately deduce from $(4.1 \mathrm{~b})$ that the sequences $u_{n}, \bar{u}_{n}$, and $\underline{u}_{n}$ have the same limit in $L^{\infty}\left(0, T ; H_{\mathrm{D}}^{1}\left(\Omega ; \mathbb{R}^{d}\right)\right)$, and the pointwise weak convergences (4.1d) ensue. Furthermore, due to estimate (3.32e), by compactness, there exists a further subsequence such that $\dot{u}_{n} \rightarrow \dot{u}$ in $\mathrm{BV}\left([0, T] ; W_{\mathrm{D}}^{1, \gamma}\left(\Omega ; \mathbb{R}^{d}\right)^{*}\right)$ as well as $\dot{u}_{n}(t) \rightarrow \dot{u}(t)$ in $W_{\mathrm{D}}^{1, \gamma}\left(\Omega ; \mathbb{R}^{d}\right)^{*}$ for all $t \in[0, T]$. Thanks to $(3.32 \mathrm{~d})$, arguing by contradiction and using that $L^{2}\left(\Omega ; \mathbb{R}^{d}\right)$ is dense in $W_{\mathrm{D}}^{1, \gamma}\left(\Omega ; \mathbb{R}^{d}\right)^{*}$, we may also conclude that $\dot{u}_{n}(t) \rightarrow \dot{u}(t)$ in $L^{2}\left(\Omega ; \mathbb{R}^{d}\right)$ for all $t \in[0, T]$, i.e. (4.1e).

Convergence of the damage variables: From estimates (3.32f) on the $\mathcal{R}_{1}$-total variation of $\left(\bar{z}_{n}\right)_{n}$ (by monotonicity of $\bar{z}_{n}$ ), combined with (3.32h), a generalized version of Helly's selection principle, cf. e.g. [51, Theorem 6.1], allows us to extract a subsequence such that $\bar{z}_{n}(t) \rightarrow z(t)$ and $\underline{z}_{n}(t) \rightarrow \underline{z}(t)$ weakly in $W^{1, q}(\Omega)$ for all $t \in[0, T]$, and $z, z \in L^{\infty}\left(0, T ; W^{1, q}(\Omega)\right)$. Moreover, the limit functions $z$ and $z$ inherit the monotonicity in time from $\bar{z}_{n}$ and $\underline{z}_{n}$, hence $z, \underline{z}_{-} \in \mathrm{BV}\left([0, T] ; L^{1}(\Omega)\right)$, and their jump sets $J$

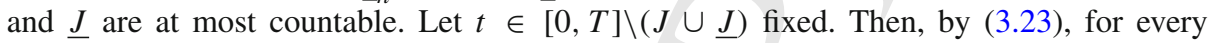
$n \in \overline{\mathbb{N}}$ we have $\bar{z}_{n}\left(t-\tau_{n}\right)=\underline{z}_{n}(t)$ and therefore as $n \rightarrow \infty$ we get $z(t)=\underline{z}(t)$. Let now $t \in J \cup \underline{J}$ and let $\left(t_{j}^{-}\right)_{j},\left(t_{j}^{+}\right)_{j} \subset[0, T] \backslash(J \cup \underline{J})$ be such that $t_{j}^{-} \nearrow t$ and $t_{j}^{+} \searrow t$. Since $z$ and $\underline{z}$ coincide on $[0, T] \backslash(J \cup \underline{J})$, we deduce that the left and the right limit satisfy $z^{-}(t)=\lim _{j} z\left(t_{j}^{-}\right)=\lim _{j} \underline{z}\left(t_{j}^{-}\right)=\underline{z}^{-}(t)$ and $z^{+}(t)=\lim _{j} z\left(t_{j}^{+}\right)=\lim _{j} \underline{z}\left(t_{j}^{+}\right)=\underline{z}^{+}(t)$. Therefore $J=\underline{J}$ and the convergences (4.1f), (4.1g), (4.1i) hold. From this, using (3.32g) we conclude that $(4.1 \mathrm{~h})$ and $(4.1 \mathrm{j})$ hold true as well. In this line, we conclude by observing that (4.1o) follows from the fact that $\int_{\Omega}\left(z_{n}(0)-z_{n}(T)\right) \mathrm{d} x$, i.e. the total variation of $\dot{z}_{n}$ on $[0, T] \times \bar{\Omega}$, converges to the total variation $\int_{\Omega}(z(0)-z(T)) \mathrm{d} x$ of $\dot{z}$, also relying on the argument from [57, Proposition 4.3, proof of (4.80)].

Convergence of the temperature variables: Due to estimate (3.32j) we have $\bar{\theta}_{n} \rightarrow \theta$ in $L^{2}\left(0, T ; H^{1}(\Omega)\right)$. Exploiting the definition of the interpolants (3.23), similarly to the arguments for the damage variables, we conclude that also $\underline{\theta}_{n} \rightarrow \theta$ in $L^{2}\left(0, T ; H^{1}(\Omega)\right)$, thus $(4.1 \mathrm{k})$ is proven. From this, convergences $(4.11)$ and $(4.1 \mathrm{~m})$ for $\left(\bar{\theta}_{n}, \underline{\theta}_{n}\right)_{n}$ follow by a generalized Aubin-Lions Lemma, cf. [60, Corollary 7.9, p. 196], making use of the estimates (3.32j), (3.32k), and (3.32l). Taking into account that $\left|\theta_{n}(t, x)\right| \leq \max \left\{\left|\bar{\theta}_{n}(t, x)\right|,\left|\underline{\theta}_{n}(t, x)\right|\right\}$ for almost all $(t, x) \in(0, T) \times \Omega$, (a generalized version of) the Lebesgue Theorem yields convergence $(4.1 \mathrm{~m})$ for $\left(\theta_{n}\right)_{n}$ as well. All in all, we conclude the weak convergence $(4.1 \mathrm{k})$, as well as (4.11), for $\left(\theta_{n}\right)_{n}$. Convergence (4.1n) is a consequence of [51, Theorem 6.1]. The positivity properties (2.14) and (2.16) (under the additional (2.15)) then follow from their discrete analogues (3.4) and (3.5), respectively, combined with (3.32k).

The fact that the limit triple $(u, z, \theta)$ is an energetic solution of the limit problem will be verified in Sects. 4.1-4.3 right below. For this, in Sect. 4.1, we first pass from time-discrete to continuous in the weak momentum balance (3.27b) using suitably chosen time-discrete test functions and deduce a time-continuous limit inequality for the mechanical energy balance (3.26) by lower semicontinuity arguments. Secondly, in Sect. 4.2 we pass to the limit in the semistability inequality (3.27a) using mutual recovery sequences. As a further step in Sect. 4.3 it has to be verified that the limit triple $(u, z, \theta)$ indeed satisfies the mechanical energy balance as an equality by deducing the reverse inequality from the momentum balance and 
the semistability so far obtained. This result allows us to conclude the convergence of the viscous dissipation terms, which, in turn, is crucial for the limit passage in the heat equation $(3.27 \mathrm{e})$.

Altogether, these steps amount to the following

Proposition 4.2 (Energetic solution of the limit problem) Let the assumptions of Theorem 2.6 be satisfied and let $(u, z, \theta)$ be a triple of regularity (2.11) obtained as a limit, in the sense of convergences (4.1), of a sequence of solutions to Problem 3.1. Then, $(u, z, \theta)$ is an energetic solution of the time-continuous problem (1.1), supplemented with the boundary conditions (1.3), in the sense of Definition 2.3.

Proof The statement of the proposition follows directly by combining Propositions 4.3, 4.6, and 4.9 and Theorem 4.5 .

\subsection{Limit Passage in the Momentum Balance and the Energy Inequalities}

Based on the convergence properties (4.1) we now pass from time-discrete to time-continuous in the weak momentum balance. By lower semicontinuity we will then carry out the limit passage in the mechanical as well as in the total energy inequality and obtain their analogues for the limit problem.

Let us mention in advance that, while the passage to the limit in most of the terms of the momentum balance can be treated in a straightforward way by exploiting the convergence properties (4.1), the quadratic terms arising from the stored elastic energy and the viscous dissipation, which involve the state-dependent coefficients $\mathbb{D}\left(\underline{z}_{n}, \underline{\theta}_{n}\right)$ and $\mathbb{C}\left(\bar{z}_{n}\right)$, need special attention. For these terms the limit will be deduced by exploiting the $L^{\infty}$-bounds (2.3) on $\mathbb{C}$ and $\mathbb{D}$ and the dominated convergence theorem.

Proposition 4.3 (Limit passage in the weak momentum balance) Let the assumptions of Theorem 2.6 be satisfied. Then, a limit triple $(u, z, \theta)$ extracted as in Proposition 4.1 solves the time-continuous momentum balance $(2.12 \mathrm{~b})$ at every $t \in[0, T]$. In particular, it holds $\dot{u} \in H^{1}\left(0, T ; H_{\mathrm{D}}^{1}\left(\Omega ; \mathbb{R}^{d}\right)^{*}\right) \cap \mathrm{C}_{\text {weak }}^{0}\left([0, T] ; L^{2}\left(\Omega ; \mathbb{R}^{d}\right)\right)$.

Proof Let $v \in L^{2}\left(0, T ; H_{\mathrm{D}}^{1}\left(\Omega ; \mathbb{R}^{d}\right)\right) \cap H^{1}\left(0, T ; L^{2}\left(\Omega ; \mathbb{R}^{d}\right)\right)$ be a test function for $(2.12 \mathrm{~b})$. It follows from, e.g., [10, p. 56, Corollary 2] and [60, p. 189, Lemma 7.2], that for every $\varepsilon>0$ there exists

$$
\begin{aligned}
& v^{\star} \in L^{2}\left(0, T ; \mathrm{C}^{1}\left(\bar{\Omega} ; \mathbb{R}^{d}\right)\right) \cap L^{2}\left(0, T ; H_{\mathrm{D}}^{1}\left(\Omega ; \mathbb{R}^{d}\right)\right) \cap H^{1}\left(0, T ; L^{2}\left(\Omega ; \mathbb{R}^{d}\right)\right): \\
& \left\|v-v^{\star}\right\|_{L^{2}\left(0, T ; H_{\mathrm{D}}^{1}\left(\Omega ; \mathbb{R}^{d}\right)\right) \cap H^{1}\left(0, T ; L^{2}\left(\Omega ; \mathbb{R}^{d}\right)\right)} \leq \varepsilon \text { and } v^{\star}=v \text { on } \partial_{\mathrm{D}} \Omega \text { in the trace sense. }
\end{aligned}
$$

In particular, $v^{\star} \in L^{2}\left(0, T ; W^{1, \gamma}\left(\Omega ; \mathbb{R}^{d}\right)\right)$, with $\gamma>4$ the same exponent as in the regularizing term $-\tau_{n} \operatorname{div}\left(|e(u)|^{\gamma-2} e(u)\right)$ in time-discrete momentum balance (3.27b). Therefore, the discrete test functions $\left(v^{\star}\right)_{n}^{k}:=\frac{1}{\tau_{n}} \int_{t_{n}^{k-1}}^{t_{n}^{k}} v^{\star}(s) \mathrm{d} s$ for all $k=0, \ldots, n$ fulfill $\left(v^{\star}\right)_{n}^{k} \in$ $W^{1, \gamma}\left(\Omega ; \mathbb{R}^{d}\right)$, so that they are admissible test functions for $(3.27 \mathrm{~b})$. We now consider the piecewise constant and linear interpolants $\bar{v}_{n}^{\star}$ and $v_{n}^{\star}$ of the elements $\left(\left(v^{\star}\right)_{n}^{k}\right)_{k=0}^{n}$. In view of (4.2), it can be checked that

$$
\begin{aligned}
& \bar{v}_{n}^{\star} \rightarrow v^{\star} \text { in } L^{2}\left(0, T ; H_{\mathrm{D}}^{1}\left(\Omega ; \mathbb{R}^{d}\right)\right) \text { and } v_{n}^{\star} \rightarrow v^{\star} \text { in } H^{1}\left(0, T ; L^{2}\left(\Omega ; \mathbb{R}^{d}\right)\right), \\
& \tau_{n}^{1 / \gamma}\left\|e\left(\bar{v}_{n}^{\star}\right)\right\|_{L^{\gamma}\left(0, T ; L^{\gamma}\left(\Omega ; \mathbb{R}^{d \times d}\right)\right)} \rightarrow 0 .
\end{aligned}
$$

Observe that (4.3a) implies

$$
v_{n}^{\star}(t) \rightarrow v^{*}(t) \text { in } L^{2}\left(\Omega ; \mathbb{R}^{d}\right) \text { for all } t \in[0, T] .
$$


Using such sequences $\left(\bar{v}_{n}^{\star}, v_{n}^{\star}\right)_{n}$ of interpolants of smooth, dense test functions, we can now carry out the limit passage in (3.27b). By the convergence properties of the given data (3.24a) and for the smooth test functions (4.3), together with the convergence results (4.1e), (4.1b) and $(4.1 \mathrm{k})$ we immediately find

$$
\begin{aligned}
& \rho \int_{\Omega}\left(\dot{u}_{n}(t) \cdot v_{n}^{\star}(t)-\dot{u}_{0} \cdot v_{n}^{\star}(0)\right) \mathrm{d} x \\
& \quad-\int_{0}^{\bar{\tau}_{n}(t)}\left(\int_{\Omega}\left(\rho \dot{u}_{n}\left(s-\tau_{n}\right) \cdot \dot{v}_{n}^{\star}-\bar{\theta}_{n} \mathbb{B}: e\left(\bar{v}_{n}^{\star}\right)\right) \mathrm{d} x-\left\langle\bar{f}_{n}, \bar{v}_{n}^{\star}\right\rangle_{H_{\mathrm{D}}^{1}\left(\Omega ; \mathbb{R}^{d}\right)}\right) \mathrm{d} s \\
& \quad-\rho \int_{\Omega}\left(\dot{u}(t) \cdot v^{\star}(t)-\dot{u}_{0} \cdot v^{\star}(0)\right) \mathrm{d} x \\
& \quad-\int_{0}^{t}\left(\int_{\Omega}\left(\rho \dot{u} \cdot \dot{v}^{\star}-\theta \mathbb{B}: e\left(v^{\star}\right)\right) \mathrm{d} x-\left\langle f, v^{\star}\right\rangle_{H_{\mathrm{D}}^{1}\left(\Omega ; \mathbb{R}^{d}\right)}\right) \mathrm{d} s .
\end{aligned}
$$

Moreover, the convergence of the term involving the $\gamma$-Laplacian follows from the estimate

$$
\begin{aligned}
& \left.\left|\int_{0}^{t} \int_{\Omega} \tau_{n}\right| e\left(\bar{u}_{n}\right)\right|^{\gamma-2} e\left(\bar{u}_{n}\right): e\left(\bar{v}_{n}^{\star}\right) \mathrm{d} x \mathrm{~d} s \mid \\
& \quad \leq \tau_{n}^{\frac{\gamma-1}{\gamma}}\left\|e\left(\bar{u}_{n}\right)\right\|_{L^{\gamma}\left((0, T) \times \Omega ; \mathbb{R}^{d \times d}\right)}^{\gamma-1} \tau_{n}^{\frac{1}{\gamma}}\left\|e\left(\bar{v}_{n}^{\star}\right)\right\|_{L^{\gamma}\left((0, T) \times \Omega ; \mathbb{R}^{d \times d}\right)} \rightarrow 0,
\end{aligned}
$$

due to the uniform bound (3.32b) and the convergence of $\left(v_{n}^{\star}\right)_{n}$ by (4.3).

Finally, in order to handle the remaining quadratic terms with state-dependent coefficients in $(3.27 \mathrm{~b})$, we will prove that

$$
\left(\mathbb{D}\left(\underline{z}_{n}, \underline{\theta}_{n}\right)+\mathbb{C}\left(\bar{z}_{n}\right)\right) e\left(\bar{v}_{n}^{\star}\right) \rightarrow(\mathbb{D}(z, \theta)+\mathbb{C}(z)) e\left(v^{\star}\right) \text { strongly in } L^{2}\left((0, T) \times \Omega ; \mathbb{R}^{d \times d}\right) .
$$

Then, the convergence of the quadratic terms with state-dependent coefficients follows from weak-strong convergence, using that both $e\left(\dot{u}_{n}\right) \rightarrow e(\dot{u})$ and $e\left(u_{n}\right) \rightarrow e(u)$ weakly in $L^{2}\left(0, T ; L^{2}\left(\Omega ; \mathbb{R}^{d \times d}\right)\right)$ by $(4.1 \mathrm{~b})$. Now, to verify (4.4) we are going to apply the dominated convergence theorem. For this, we observe that for a.e. $t \in(0, T)$ we have $\left|\left(\mathbb{D}\left(\underline{z}_{n}(t), \underline{\theta}_{n}(t)\right)+\mathbb{C}\left(\bar{z}_{n}(t)\right)\right): e\left(\bar{v}_{n}^{\star}(t)\right)\right| \rightarrow|(\mathbb{D}(z(t), \theta(t))+\mathbb{C}(z(t))): e(v(t))|$ pointwise a.e. in $\Omega$, by assumption (2.3b) and since by convergence results (4.1j) and (4.11) we can resort to a subsequence $\left(\underline{z}_{n}(t), \bar{z}_{n}(t), \underline{\theta}_{n}\right)_{n}$ that converges pointwise a.e. in $\Omega$ for a.e. $t \in(0, T)$. Moreover, by assumption (2.3) we find an integrable, convergent majorant, i.e.,

$$
\left|\left(\mathbb{D}\left(\underline{z}_{n}, \underline{\theta}_{n}\right)+\mathbb{C}\left(\bar{z}_{n}\right)\right) e\left(\bar{v}_{n}^{\star}\right)\right| \leq\left(C_{\mathbb{D}}^{2}+C_{\mathbb{C}}^{2}\right)\left|e\left(\bar{v}_{n}^{\star}\right)\right| \rightarrow\left(C_{\mathbb{D}}^{2}+C_{\mathbb{C}}^{2}\right)\left|e\left(v^{\star}\right)\right|
$$

pointwise a.e. in $(0, T) \times \Omega$ and with respect to the strong $\left.L^{2}((0, T) \times \Omega)\right)$-topology by (4.3). Hence, a generalized version of the Dominated Convergence Theorem, cf. e.g., [55, Sect. 4.4, Theorem 19], yields (4.4). This concludes the limit passage in the momentum balance for smooth test function as in (4.2). By density this result carries over to all test functions $v \in L^{2}\left(0, T ; H_{\mathrm{D}}^{1}\left(\Omega ; \mathbb{R}^{d}\right)\right) \cap H^{1}\left(0, T ; L^{2}\left(\Omega ; \mathbb{R}^{d}\right)\right)$. As by $(4.1 \mathrm{e})$ we have $\dot{u}(t) \in L^{2}\left(\Omega ; \mathbb{R}^{d}\right)$ for every $t \in[0, T]$, we immediately deduce that (2.12b) holds true at all $t \in[0, T]$.

The last assertion follows from Remark 2.5.

Lemma 4.4 (Energy inequalities by lower semicontinuity) Let the assumptions of Theorem 2.6 be satisfied and let $(u, z, \theta)$ be a limit triple given by Proposition 4.1. Then for every $t \in[0, T]$ we have 


$$
\begin{aligned}
& \frac{\rho}{2} \int_{\Omega}|\dot{u}(t)|^{2} \mathrm{~d} x+\mathcal{E}(t, u(t), z(t))+\int_{\Omega}\left(z(t)-z_{0}\right) \mathrm{d} x+\int_{0}^{t} \int_{\Omega}(\mathbb{D}(z, \theta) e(\dot{u})-\theta \mathbb{B}): e(\dot{u}) \mathrm{d} x \mathrm{~d} s \\
& \quad \leq \frac{\rho}{2} \int_{\Omega}\left|\dot{u}_{0}\right|^{2} \mathrm{~d} x+\mathcal{E}\left(0, u_{0}, z_{0}\right)-\int_{0}^{t}\langle\dot{f}, v\rangle_{H_{\mathrm{D}}^{1}\left(\Omega ; \mathbb{R}^{d}\right)} \mathrm{d} s .
\end{aligned}
$$

Proof It is enough to pass to the limit in (3.27c) taking into account (3.24b), (4.1d), (4.1e), (4.1j), and (4.11).

\subsection{Limit Passage in the Semistability Inequality}

In order to carry out the passage from time-discrete to continuous in the semistability inequality we follow the well-established method of circumventing a direct passage to the limit on the left- and on the right-hand side of the semistability inequality (3.27a). Instead, it is enough to prove a limsup inequality for the difference, cf. also [44,47], using a so-called mutual recovery sequence. This procedure, which allows one to take advantage of some cancelations in the regularizing terms for the internal variable $\mathcal{G}(z, \nabla z)$, has been already employed in $[44,67,68]$ in problems concerned with (fully) rate-independent, partial, isotropic and unidirectional damage, featuring a $W^{1, q}(\Omega)$-gradient regularization, with $q>d$ in [44], any $q>1$ in [68] as in the present context, and $q=1$ in [67]. In what follows, we verify that the recovery sequence constructed in [68], where $\mathcal{G}(z, \nabla z)=|\nabla z|^{q}$, is also suited in our setting of semistability with a general gradient term.

More precisely, let us fix $t \in[0, T]$ in the energy functionals $\mathcal{E}_{n}$ from (3.26), and a sequence $\left(v_{n}, \zeta_{n}\right)_{n} \subset H_{\mathrm{D}}^{1}\left(\Omega ; \mathbb{R}^{d}\right) \times z$ such that

$$
\begin{aligned}
& v_{n} \rightarrow v \quad \text { weakly in } H_{\mathrm{D}}^{1}\left(\Omega ; \mathbb{R}^{d}\right), \quad \zeta_{n} \rightarrow \zeta \quad \text { weakly in } W^{1, q}(\Omega), \\
& \varepsilon_{n}\left(t, v_{n}, \zeta_{n}\right) \leq \mathcal{E}_{n}\left(t, v_{n}, \hat{\zeta}\right)+\mathcal{R}_{1}\left(\hat{\zeta}-\zeta_{n}\right) \quad \text { for all } \hat{\zeta} \in Z,
\end{aligned}
$$

i.e., $\zeta_{n}$ is semistable for $\mathcal{E}_{n}\left(t, v_{n}, \cdot\right)$. Given $\tilde{\zeta} \in z$ let the recovery sequence $\left(\tilde{\zeta}_{n}\right)_{n} \subset z$ be defined by

$$
\tilde{\zeta}_{n}:=\min \left\{\zeta_{n}, \max \left\{\left(\tilde{\zeta}-\delta_{n}, 0\right)\right\}\right\}= \begin{cases}\left(\tilde{\zeta}-\delta_{n}\right) & \text { on } A_{n}=\left\{0 \leq\left(\tilde{\zeta}-\delta_{n}\right) \leq \zeta_{n}\right\}, \\ \zeta_{n} & \text { on } B_{n}=\left\{\tilde{\zeta}-\delta_{n}>\zeta_{n}\right\}, \\ 0 & \text { on } C_{n}=\left\{\tilde{\zeta}-\delta_{n}<0\right\},\end{cases}
$$

where $\delta_{n}:=\left\|\zeta_{n}-\zeta\right\|_{L^{q}(\Omega)}^{1 / q}$.

The sequence $\left(\tilde{\zeta}_{n}\right)_{n}$ was introduced in [68] where it was shown that

$$
\tilde{\zeta}_{n} \rightarrow \tilde{\zeta} \text { in } W^{1, q}(\Omega) \text { for } q \in(1, \infty) \text { from }(2.5 d) \text { fixed. }
$$

Note however that strong convergence in $W^{1, q}(\Omega)$ cannot be expected, since $\zeta_{n} \rightarrow \zeta$ weakly in $W^{1, q}(\Omega)$, only. This makes it impossible to show directly that $\mathcal{G}\left(\tilde{\zeta}_{n}, \nabla \tilde{\zeta}_{n}\right) \rightarrow \mathcal{G}(\tilde{\zeta}, \nabla \tilde{\zeta})$, since this would require the strong convergence of the gradients. Nevertheless the following result holds.

Theorem 4.5 Let the assumptions of Theorem 2.6 be satisfied. Let $t \in[0, T]$ be fixed and consider a sequence $\left(v_{n}, \zeta_{n}\right)_{n} \subset H_{\mathrm{D}}^{1}\left(\Omega ; \mathbb{R}^{d}\right) \times \mathcal{Z}$ such that (4.6) holds. Given $\tilde{\zeta} \in \mathcal{Z}$, let $\left(\tilde{\zeta}_{n}\right)_{n} \subset Z$ as in (4.7). Then

$$
0 \leq \limsup _{n \rightarrow \infty}\left(\varepsilon_{n}\left(t, v_{n}, \tilde{\zeta}_{n}\right)-\varepsilon_{n}\left(t, v_{n}, \zeta_{n}\right)+\mathcal{R}_{1}\left(\tilde{\zeta}_{n}-\zeta_{n}\right)\right) \leq \mathcal{E}(t, v, \tilde{\zeta})-\mathcal{E}(t, v, \zeta)+\mathcal{R}_{1}(\tilde{\zeta}-\zeta)
$$


Therefore the limit $\zeta$ is semistable for $\mathcal{E}(t, v, \cdot)$.

Proof First of all note that, if $\tilde{\zeta} \in \mathcal{Z}$ does not satisfy $0 \leq \tilde{\zeta} \leq \zeta$, then (4.9) trivially holds, since in this case $\mathcal{R}_{1}(\tilde{\zeta}-\zeta)=+\infty$.

Assume now $0 \leq \tilde{\zeta} \leq \zeta$ for a.e. $x \in \Omega$. Let us estimate the left-hand side of (4.9) as follows:

$$
\begin{aligned}
& \limsup _{n \rightarrow \infty}\left(\mathcal{E}_{n}\left(t, v_{n}, \tilde{\zeta}_{n}\right)-\mathcal{E}_{n}\left(t, v_{n}, \zeta_{n}\right)+\mathcal{R}_{1}\left(\tilde{\zeta}_{n}-\zeta_{n}\right)\right) \\
& \leq \limsup _{n \rightarrow \infty} \int_{\Omega}\left(\mathbb{C}\left(\tilde{\zeta}_{n}\right)-\mathbb{C}\left(\zeta_{n}\right)\right) e\left(v_{n}\right): e\left(v_{n}\right) \mathrm{d} x \\
& \quad+\limsup _{n \rightarrow \infty}\left(\mathcal{G}\left(\tilde{\zeta}_{n}, \nabla \tilde{\zeta}_{n}\right)-\mathcal{G}\left(\zeta_{n}, \nabla \zeta_{n}\right)\right)+\limsup _{n \rightarrow \infty} \mathcal{R}_{1}\left(\tilde{\zeta}_{n}-\zeta_{n}\right)
\end{aligned}
$$

and then treat each of the terms on the right-hand side of (4.10) separately. Since $\zeta_{n} \rightarrow \zeta$ in $W^{1, q}(\Omega)$, we may choose a (not relabeled) subsequence that converges pointwise a.e. in $\Omega$.

Estimation of $\lim \sup _{n \rightarrow \infty}\left(\mathcal{G}\left(\tilde{\zeta}_{n}, \nabla \tilde{\zeta}_{n}\right)-\mathcal{G}\left(\zeta_{n}, \nabla \zeta_{n}\right)\right)$ : Note that $G\left(\tilde{\zeta}_{n}, \nabla \tilde{\zeta}_{n}\right)=G\left(\zeta_{n}\right.$, $\left.\nabla \zeta_{n}\right)$ on $B_{n}$. If $\left\|\zeta_{n}-\zeta\right\|_{L^{q}(\Omega)}>0$, by Markov's inequality

$$
\mathcal{L}^{d}\left(B_{n}\right) \leq \mathcal{L}^{d}\left(\left[\delta_{n} \leq\left|\zeta_{n}-\zeta\right|\right]\right) \leq \frac{1}{\delta_{n}} \int_{\Omega}\left|\zeta_{n}-\zeta\right| \mathrm{d} x \leq \frac{1}{\delta_{n}}\left\|\zeta_{n}-\zeta\right\|_{L^{q}(\Omega)} \rightarrow 0,
$$

with $\delta_{n}$ from (4.7), while for $\left\|\zeta_{n}-\zeta\right\|_{L^{q}(\Omega)}=0$ it is indeed $\mathcal{L}^{d}\left(B_{n}\right)=0$, thus

$$
\mathcal{L}^{d}\left(A_{n} \cup C_{n}\right) \rightarrow \mathcal{L}^{d}(\Omega) .
$$

In what follows, $X_{D}$ will denote the characteristic function of a set $D$. By (2.5b), (2.5d) and (4.7), we deduce

$$
\begin{aligned}
\limsup _{n \rightarrow \infty} & \left(\mathcal{G}\left(\tilde{\zeta}_{n}, \nabla \tilde{\zeta}_{n}\right)-\mathcal{G}\left(\zeta_{n}, \nabla \zeta_{n}\right)\right) \\
= & \limsup _{n \rightarrow \infty} \int_{A_{n}} G\left(\left(\tilde{\zeta}-\delta_{n}\right), \nabla \tilde{\zeta}\right) \mathrm{d} x+\int_{C_{n}} G(0,0) \mathrm{d} x-\int_{A_{n} \cup C_{n}} G\left(\zeta_{n}, \nabla \zeta_{n}\right) \mathrm{d} x \\
\leq & \limsup _{n \rightarrow \infty}\left(\int_{\Omega} G\left(X_{A_{n}}\left(\tilde{\zeta}-\delta_{n}\right), X_{A_{n}} \nabla \tilde{\zeta}\right) \mathrm{d} x+\int_{\Omega} G\left(0, X_{C_{n}} \nabla \tilde{\zeta}\right) \mathrm{d} x\right. \\
& \left.-\int_{\Omega} G\left(X_{A_{n} \cup C_{n}} \zeta_{n}, X_{A_{n} \cup C_{n}} \nabla \zeta_{n}\right) \mathrm{d} x\right) \\
= & \limsup _{n \rightarrow \infty}\left(\int_{\Omega} G\left(X_{A_{n} \cup C_{n}}\left(\tilde{\zeta}_{n}\right), X_{A_{n} \cup C_{n}} \nabla \tilde{\zeta}\right) \mathrm{d} x-\int_{\Omega} G\left(X_{A_{n} \cup C_{n}} \zeta_{n}, X_{A_{n} \cup C_{n}} \nabla \zeta_{n}\right) \mathrm{d} x\right) \\
\leq & \mathcal{G}(\tilde{\zeta}, \nabla \tilde{\zeta})-\liminf _{n \rightarrow \infty} \mathcal{G}\left(X_{A_{n} \cup C_{n}} \zeta_{n}, X_{A_{n} \cup C_{n}} \nabla \zeta_{n}\right) \\
\leq & \mathcal{G}(\tilde{\zeta}, \nabla \tilde{\zeta})-\mathcal{G}(\zeta, \nabla \zeta),
\end{aligned}
$$

where in the second integral term in the third line we have used the obvious identity $\chi_{C_{n}} 0=0$. To obtain (4.12a) we have used the dominated convergence theorem, while in order to prove (4.12b) we employed the lower semicontinuity of $\mathcal{G}: L^{q}(\Omega) \times L^{q}\left(\Omega ; \mathbb{R}^{d}\right) \rightarrow \mathbb{R} \cup\{\infty\}$, since, by (4.8) and (4.11), we have $X_{A_{n} \cup C_{n}} \zeta_{n} \rightarrow \zeta$ strongly in $L^{q}(\Omega)$ and $X_{A_{n} \cup C_{n}} \nabla \zeta_{n} \rightarrow \nabla \zeta$ weakly in $L^{q}\left(\Omega ; \mathbb{R}^{d}\right)$.

Estimation of the remaining terms in (4.10): Since construction (4.7) ensures $\tilde{\zeta}_{n} \leq \zeta_{n}$ for every $n \in \mathbb{N}$, as well as $\tilde{\zeta}_{n} \rightarrow \tilde{\zeta}$ in $L^{q}(\Omega)$, due to $\zeta_{n} \rightarrow \zeta$ in $L^{q}(\Omega)$, we immediately conclude that $\mathcal{R}_{1}\left(\tilde{\zeta}_{n}-\zeta_{n}\right) \rightarrow \mathcal{R}_{1}(\tilde{\zeta}-\zeta)$.

We now estimate the difference of the quadratic terms in the mechanical energy. As $\tilde{\zeta}_{n} \leq$ $\zeta_{n}$, by the monotonicity assumption (2.4) we have that $\left(\mathbb{C}\left(\tilde{\zeta}_{n}\right)-\mathbb{C}\left(\zeta_{n}\right)\right) e\left(v_{n}\right): e\left(v_{n}\right) \leq 0$. 
Since both $\zeta_{n} \rightarrow \zeta$ and $\tilde{\zeta}_{n} \rightarrow \tilde{\zeta}$ in $L^{q}(\Omega)$, the Lipschitz-continuity of $\mathbb{C}$, cf. (2.3b), implies that $\mathbb{C}\left(\tilde{\zeta}_{n}\right)-\mathbb{C}\left(\zeta_{n}\right) \rightarrow(\mathbb{C}(\tilde{\zeta})-\mathbb{C}(\zeta))$ in $L^{q}\left(\Omega ; \mathbb{R}_{\text {sym }}^{d \times d \times d \times d}\right)$. Let us consider the auxiliary functional $\mathcal{C}: L^{q}(\Omega) \times L^{q}(\Omega) \times L^{2}\left(\Omega ; \mathbb{R}^{d \times d}\right) \rightarrow \mathbb{R}$ defined by

$$
\mathcal{C}(\zeta, \tilde{\zeta}, e):=\int_{\Omega}(\mathbb{C}(\zeta(x))-\mathbb{C}(\min \{\zeta(x), \tilde{\zeta}(x)\})) e(x): e(x) \mathrm{d} x
$$

By e.g. [21, Theorem 7.5, p. 492] the functional $\mathcal{C}$ is lower semicontinuous with respect to the strong convergence in $L^{q}(\Omega) \times L^{q}(\Omega)$ and the weak convergence in $L^{2}\left(\Omega ; \mathbb{R}^{d \times d}\right)$. Thus, the first term on the right-hand side of (4.10) can be rewritten and estimated as follows, using (3.32c) and the lower semicontinuity of $\mathcal{C}$,

$$
\limsup _{n \rightarrow \infty} \int_{\Omega}\left(\mathbb{C}\left(\tilde{\zeta}_{n}\right)-\mathbb{C}\left(\zeta_{n}\right)\right) e\left(v_{n}\right): e\left(v_{n}\right) \mathrm{d} x \leq \int_{\Omega}(\mathbb{C}(\tilde{\zeta})-\mathbb{C}(\zeta)) e(v): e(v) \mathrm{d} x
$$

Combining the above established estimates for the three terms on the right-hand side of (4.10) shows that condition (4.9) is satisfied.

\subsection{Energy Equalities and Limit Passage in the Heat Equation}

We now show that the limit triple $(u, z, \theta)$ satisfies the mechanical energy equality (2.12c). The inequality $(\leq)$ has been proven in Lemma 4.4. The opposite inequality is found by approximation with Riemann sums, as common in existence proofs of rate-independent and rate-dependent evolutions, see e.g. [13].

Proposition 4.6 (Mechanical energy equality) Let the assumptions of Theorem 2.6 be satisfied, let $(u, z, \theta)$ be a triple given by Proposition 4.1, and let $t \in[0, T]$. Then $(2.12 c)$ holds.

Proof We fix a sequence of subdivisions $\left(s_{n}^{k}\right)_{0 \leq k \leq k_{n}}$ of the interval $[0, t]$, with $0=s_{n}^{0}<$ $s_{n}^{1}<\cdots<s_{n}^{k_{n}-1}<s_{n}^{k_{n}}=t, \lim _{n} \max _{k}\left(s_{n}^{k}-s_{n}^{k-1}\right)=0$, and

$$
\left|\sum_{k=1}^{k_{n}} \int_{s_{n}^{k-1}}^{s_{n}^{k}} \int_{\Omega}\left[\mathbb{C}\left(z\left(s_{n}^{k}\right)\right)-\mathbb{C}(z(s))\right] e(u(s)): e(\dot{u}(s)) \mathrm{d} x \mathrm{~d} s\right| \rightarrow 0 .
$$

The existence of such a sequence is guaranteed by [27], see also [57, Proposition 4.3, Step 7]. Taking $z\left(s_{n}^{k}\right)$ as test function in the time-continuous semistability inequality (2.12a) at time $s_{n}^{k-1}$ we get

$$
\begin{aligned}
& \mathcal{E}\left(s_{n}^{k-1}, u\left(s_{n}^{k-1}\right), z\left(s_{n}^{k-1}\right)\right) \\
& \quad \leq \mathcal{E}\left(s_{n}^{k-1}, u\left(s_{n}^{k-1}\right), z\left(s_{n}^{k}\right)\right)+\int_{\Omega}\left(z\left(s_{n}^{k-1}\right)-z\left(s_{n}^{k}\right)\right) \mathrm{d} x \\
& \quad=\mathcal{E}\left(s_{n}^{k}, u\left(s_{n}^{k}\right), z\left(s_{n}^{k}\right)\right)+\int_{\Omega}\left(z\left(s_{n}^{k-1}\right)-z\left(s_{n}^{k}\right)\right) \mathrm{d} x-\int_{s_{n}^{k-1}}^{s_{n}^{k}} \partial_{t} \mathcal{E}(s, u(s), z(s)) \mathrm{d} s \\
& \quad+\int_{s_{n}^{k-1}}^{s_{n}^{k}}\langle f(s), \dot{u}(s)\rangle_{H_{\mathrm{D}}^{1}\left(\Omega ; \mathbb{R}^{d}\right)} \mathrm{d} s-\int_{s_{n}^{k-1}}^{s_{n}^{k}} \int_{\Omega} \mathbb{C}\left(z\left(s_{n}^{k}\right)\right) e(u(s)): e(\dot{u}(s)) \mathrm{d} x \mathrm{~d} s .
\end{aligned}
$$


Next we sum up the previous inequality over $k=1, \ldots, k_{n}$ and we pass to the limit in $n$ in the last term thanks to (4.13), obtaining

$$
\begin{aligned}
\mathcal{E}\left(0, u_{0}, z_{0}\right) \leq & \mathcal{E}(t, u(t), z(t))+\int_{\Omega}\left(z_{0}-z(t)\right) \mathrm{d} x-\int_{0}^{t} \partial_{t} \mathcal{E}(s, u(s), z(s)) \mathrm{d} s \\
& +\int_{0}^{t}\langle f(s), \dot{u}(s)\rangle_{H_{\mathrm{D}}^{1}\left(\Omega ; \mathbb{R}^{d}\right)} \mathrm{d} s-\int_{0}^{t} \int_{\Omega} \mathbb{C}(z(s)) e(u(s)): e(\dot{u}(s)) \mathrm{d} x \mathrm{~d} s .
\end{aligned}
$$

Further, thanks to Remark 2.5 we can test $(2.12 \mathrm{~b})$ by $\dot{u}$ and get

$$
\begin{aligned}
& \frac{\rho}{2}\|\dot{u}(t)\|_{L^{2}\left(\Omega ; \mathbb{R}^{d}\right)}^{2}+\int_{0}^{t} \int_{\Omega}(\mathbb{D}(z, \theta) e(\dot{u})+\mathbb{C}(z) e(u)-\theta \mathbb{B}): e(\dot{u}) \mathrm{d} x \mathrm{~d} s \\
& =\frac{\rho}{2}\left\|\dot{u}_{0}\right\|_{L^{2}\left(\Omega ; \mathbb{R}^{d}\right)}^{2}+\int_{0}^{t}\langle f, \dot{u}\rangle_{H_{\mathrm{D}}^{1}\left(\Omega ; \mathbb{R}^{d}\right)} \mathrm{d} s,
\end{aligned}
$$

where we applied the by-part integration formula (2.13), as allowed by [60, Lemma 7.3].

Summing up (4.15) with (4.14) we obtain

$$
\begin{aligned}
\mathcal{E}\left(0, u_{0}, z_{0}\right) \leq & \mathcal{E}(t, u(t), z(t))+\frac{\rho}{2} \int_{\Omega}|\dot{u}(t)|^{2} \mathrm{~d} x+\int_{\Omega}\left(z_{0}-z(t)\right) \mathrm{d} x \\
& -\int_{0}^{t} \partial_{t} \mathcal{E}(s, u(s), z(s)) \mathrm{d} s \\
& -\frac{\rho}{2} \int_{\Omega}\left|\dot{u}_{0}\right|^{2} \mathrm{~d} x+\int_{0}^{t} \int_{\Omega}(\mathbb{D}(z(s), \theta(s)) e(\dot{u}(s))-\theta(s) \mathbb{B}): e(\dot{u}(s)) \mathrm{d} x \mathrm{~d} s .
\end{aligned}
$$

Combining this estimate with the reverse inequality (4.5) concludes the proof of (2.12c).

In order to prove a stronger convergence of the displacements we shall repeatedly make use of the following result. Given two constants $C_{1}, C_{2}$ with $0<C_{1} \leq C_{2}$, let $\mathcal{T}_{C_{1}, C_{2}}$ denote the class of tensors $\mathbb{A} \in \mathbb{R}^{d \times d \times d \times d}$ that are symmetric, i.e.,

$$
\mathbb{A}_{i j k l}=\mathbb{A}_{j i k l}=\mathbb{A}_{i j l k}=\mathbb{A}_{k l i j},
$$

positive definite and bounded:

$$
C_{1}|A|^{2} \leq \mathbb{A} A: A \leq C_{2}|A|^{2} \quad \text { for every } A \in \mathbb{R}_{\text {sym }}^{d \times d} .
$$

\section{Lemma 4.7 Let $\mathcal{K}_{n}$ be the functional defined by}

$$
\mathcal{K}_{n}(e):=\int_{0}^{T} \int_{\Omega} \mathbb{A}_{n}(t, x) e(t, x): e(t, x) \mathrm{d} x \mathrm{~d} t \text { for every } e \in L^{2}\left((0, T) \times \Omega ; \mathbb{R}^{d \times d}\right),
$$

where $\mathbb{A}_{n} \in L^{\infty}\left((0, T) \times \Omega ; \mathcal{T}_{C_{1}, C_{2}}\right)$ are such that

$$
\begin{aligned}
\mathbb{A}_{n}(t, x) \rightarrow & \mathbb{A}_{\infty}(t, x) \text { for a.e. } t \in(0, T) \text { and a.e. } x \in \Omega, \\
e_{n} \rightarrow & e_{\infty} \text { weakly in } L^{2}\left((0, T) \times \Omega ; \mathbb{R}^{d \times d}\right), \\
& \lim \sup _{n \rightarrow \infty} \mathcal{K}_{n}\left(e_{n}\right) \leq \mathcal{K}_{\infty}\left(e_{\infty}\right),
\end{aligned}
$$

and $\mathcal{K}_{\infty}$ is defined by

$\mathcal{K}_{\infty}(e):=\int_{0}^{T} \int_{\Omega} \mathbb{A}_{\infty}(t, x) e(t, x): e(t, x) \mathrm{d} x \mathrm{~d} t$ for every $e \in L^{2}\left((0, T) \times \Omega ; \mathbb{R}^{d \times d}\right)$. 
Then, $\lim _{n \rightarrow \infty} \mathcal{K}_{n}\left(e_{n}\right)=\mathcal{K}_{\infty}\left(e_{\infty}\right)$ and

$$
e_{n} \rightarrow e_{\infty} \quad \text { strongly in } L^{2}\left((0, T) \times \Omega ; \mathbb{R}^{d \times d}\right) .
$$

Proof It is enough to observe that under the above hypotheses $\mathbb{A}_{\infty} \in L^{\infty}\left((0, T) \times \Omega ; \mathcal{T}_{C_{1}, C_{2}}\right)$ and

$$
\begin{aligned}
\mathcal{K}_{n}\left(e_{n}-e_{\infty}\right) & =\int_{0}^{T} \int_{\Omega} \mathbb{A}_{n}(t, x)\left(e_{n}(t, x)-e_{\infty}\right):\left(e_{n}(t, x)-e_{\infty}(t, x)\right) \mathrm{d} x \mathrm{~d} t \\
& =\mathcal{K}_{n}\left(e_{n}\right)-2 \int_{0}^{T} \int_{\Omega} \mathbb{A}_{n}(t, x) e_{\infty}(t, x): e_{n}(t, x) \mathrm{d} x \mathrm{~d} t+\mathcal{K}_{n}\left(e_{\infty}\right) .
\end{aligned}
$$

By (4.16) and (4.17a) we obtain $\lim \sup _{n} \mathcal{K}_{n}\left(e_{n}-e_{\infty}\right) \leq 0$. Since $\mathbb{A}_{n}(t, x) \in \mathcal{T}_{C_{1}, C_{2}}$ we have $\mathcal{K}_{n}\left(e_{n}-e_{\infty}\right) \geq C_{1}\left\|e_{n}-e_{\infty}\right\|_{L^{2}\left((0, T) \times \Omega ; \mathbb{R}^{d \times d}\right)}^{2}$, so that (4.18) holds.

Thanks to the mechanical energy inequality proven above, we may deduce strong convergence of the displacements, as provided in the following lemma.

Lemma 4.8 (Stronger convergences) Let the assumptions of Theorem 2.6 be satisfied and let $(u, z, \theta)$ be a triple given by Proposition 4.1. Then

$$
\lim _{n \rightarrow \infty} \int_{0}^{T} \int_{\Omega} \mathbb{D}\left(\underline{z}_{n}, \underline{\theta}_{n}\right) e\left(\dot{u}_{n}\right): e\left(\dot{u}_{n}\right) \mathrm{d} x \mathrm{~d} t=\int_{0}^{T} \int_{\Omega} \mathbb{D}(z, \theta) e(\dot{u}): e(\dot{u}) \mathrm{d} x \mathrm{~d} t
$$

and then

$$
e\left(\dot{u}_{n}\right) \rightarrow e(\dot{u}) \text { strongly in } L^{2}\left((0, T) \times \Omega ; \mathbb{R}^{d \times d}\right) .
$$

Proof By lower semicontinuity, taking into account the convergences already proven in Proposition 4.1, together with both the discrete mechanical energy inequality $(3.27 \mathrm{c})$ and the mechanical energy equality $(2.12 \mathrm{c})$, the following chain of inequalities holds:

$$
\begin{aligned}
& \int_{0}^{T} \int_{\Omega} \mathbb{D}(z, \theta) e(\dot{u}): e(\dot{u}) \mathrm{d} x \mathrm{~d} t+\int_{\Omega}\left(z_{0}-z(T)\right) \mathrm{d} x \\
& \leq \liminf _{n}\left(\int_{0}^{T} \int_{\Omega} \mathbb{D}\left(\underline{z}_{n}, \underline{\theta}_{n}\right) e\left(\dot{u}_{n}\right): e\left(\dot{u}_{n}\right) \mathrm{d} x \mathrm{~d} t+\int_{\Omega}\left(z_{n}(0)-z_{n}(T)\right) \mathrm{d} x\right) \\
& \leq \limsup _{n}\left(\int_{0}^{T} \int_{\Omega} \mathbb{D}\left(\underline{z}_{n}, \underline{\theta}_{n}\right) e\left(\dot{u}_{n}\right): e\left(\dot{u}_{n}\right) \mathrm{d} x \mathrm{~d} t+\int_{\Omega}\left(z_{n}(0)-z_{n}(T)\right) \mathrm{d} x\right) \\
& \leq \underset{n}{\lim \sup }\left(-\mathcal{E}_{n}\left(T, u_{n}(T), z_{n}(T)\right)+\mathcal{E}_{n}\left(0, u_{0}, z_{0}\right)-\frac{\rho}{2} \int_{\Omega}\left|\dot{u}_{n}(T)\right|^{2} \mathrm{~d} x+\frac{\rho}{2} \int_{\Omega}\left|\dot{u}_{0}\right|^{2} \mathrm{~d} x\right. \\
& \left.+\int_{0}^{T} \int_{\Omega} \bar{\theta}_{n} \mathbb{B}: e\left(\dot{u}_{n}\right) \mathrm{d} x \mathrm{~d} t+\int_{0}^{T} \partial_{t} \varepsilon_{n}\left(s, \underline{u}_{n}, \underline{z}_{n}\right) \mathrm{d} s\right) \\
& \leq-\mathcal{E}(T, u(T), z(T)))+\mathcal{E}\left(0, u_{0}, z_{0}\right)-\frac{\rho}{2} \int_{\Omega}|\dot{u}(T)|^{2} \mathrm{~d} x+\frac{\rho}{2} \int_{\Omega}\left|\dot{u}_{0}\right|^{2} \mathrm{~d} x \\
& +\int_{0}^{T} \int_{\Omega} \theta \mathbb{B}: e(\dot{u}) \mathrm{d} x \mathrm{~d} t+\int_{0}^{T} \partial_{t} \mathcal{E}(s, u, z) \mathrm{d} s \\
& =\int_{0}^{T} \int_{\Omega} \mathbb{D}(z, \theta) e(\dot{u}): e(\dot{u}) \mathrm{d} x \mathrm{~d} t+\int_{\Omega}\left(z_{0}-z(T)\right) \mathrm{d} x .
\end{aligned}
$$

Hence all inequalities above are actually equalities and we deduce that (4.19) holds.

\section{袋 Springer}


Next, we apply Lemma 4.7 with $\mathbb{A}_{n}=\mathbb{D}\left(\underline{z}_{n}, \underline{\theta}_{n}\right), \mathbb{A}_{\infty}=\mathbb{D}(z, \theta), e_{n}=e\left(\dot{u}_{n}\right)$, and $e_{\infty}=e(\dot{u})$. Indeed, (4.17a) is obtained from the strong convergences (4.1j) and (4.11) up to the passage to a further subsequence converging pointwise; the weak convergence $(4.17 \mathrm{~b})$ is given in (4.1b), while (4.17c) is provided by (4.19). Therefore we deduce that (4.20) holds (for the initial subsequence, since the limit is the same for all subsubsequences).

Finally, we pass to the limit in the heat equation.

Proposition 4.9 (Limit passage in the weak form of the heat equation) Let the assumptions of Theorem 2.6 be satisfied, Let $(u, z, \theta)$ be a triple given by Proposition 4.1, and let $t \in[0, T]$. Then the weak formulation of the heat equation (2.12d) holds.

Proof Let us fix $\eta \in H^{1}\left(0, T ; L^{2}(\Omega)\right) \cap C^{0}\left([0, T] ; W^{2, d+\delta}(\Omega)\right)$, define $\eta_{n}^{k}:=\eta\left(t_{n}^{k}\right)$ for all $k=0, \ldots, n$, and let $\eta_{n}, \bar{\eta}_{n}$ be the piecewise linear and constant interpolations of the values $\left(\eta_{n}^{k}\right)$. It can be checked that

$$
\begin{aligned}
& \bar{\eta}_{n} \rightarrow \eta \text { in } L^{p}\left(0, T ; W^{2, d+\delta}(\Omega)\right) \text { for all } 1 \leq p<\infty, \\
& \bar{\eta}_{n} \stackrel{*}{\rightarrow} \eta \text { in } L^{\infty}\left(0, T ; W^{2, d+\delta}(\Omega)\right), \\
& \eta_{n} \rightarrow \eta \quad \text { in } H^{1}\left(0, T ; L^{2}(\Omega)\right) \cap \mathrm{C}^{0}\left(0, T ; W^{2, d+\delta}(\Omega)\right) .
\end{aligned}
$$

We now pass to the limit in the discrete heat equation (3.27e) tested by $\eta_{n}$. The first three integral terms on the left-hand side of (3.27e) can be dealt with combining convergences (4.11)-(4.1n) with (4.21). In order to pass to the limit in the fourth one, we argue along the lines of [63, proof of Theorem 2.8] and derive a finer estimate for $\left(\mathbb{K}\left(\bar{z}_{n}, \bar{\theta}_{n}\right) \nabla \bar{\theta}_{n}\right)_{n}$. Indeed, thanks to $(2.6 \mathrm{~b})$ we have

$$
\left|\mathbb{K}\left(\bar{z}_{n}, \bar{\theta}_{n}\right) \nabla \bar{\theta}_{n}\right| \leq c_{2}\left(\left|\bar{\theta}_{n}\right|^{(\kappa-\alpha+2) / 2}\left|\bar{\theta}_{n}\right|^{(\kappa+\alpha-2) / 2}\left|\nabla \bar{\theta}_{n}\right|+\left|\nabla \bar{\theta}_{n}\right|\right) \quad \text { a.e. in }(0, T) \times \Omega,
$$

with $\alpha$ as in (3.37). From this particular estimate we also gather that $\left|\bar{\theta}_{n}\right|^{(\kappa+\alpha-2) / 2}\left|\nabla \bar{\theta}_{n}\right|$ is bounded in $L^{2}((0, T) \times \Omega)$. Since $\left(\bar{\theta}_{n}\right)_{n}$ is bounded in $L^{8 / 3}((0, T) \times \Omega)$ if $d=3$ (and in $L^{3}((0, T) \times \Omega)$ if $\left.d=2\right)$, choosing $\alpha \in(1 / 2,1)$ such that $\kappa-\alpha<2 / 3$ (which can be done, since $\kappa<5 / 3)$, we conclude that $\left|\bar{\theta}_{n}\right|^{(\kappa-\alpha+2) / 2}$ is bounded in $L^{2+\delta}((0, T) \times \Omega)$ for some $\delta>0$. All in all, we have that $\mathbb{K}\left(\bar{z}_{n}, \bar{\theta}_{n}\right) \nabla \bar{\theta}_{n}$ is bounded in $L^{1+\delta}\left((0, T) \times \Omega ; \mathbb{R}^{d}\right)$ for some $\delta>0$. With the very same arguments as in [63, proof of Theorem 2.8], we show that

$$
\mathbb{K}\left(\bar{z}_{n}, \bar{\theta}_{n}\right) \nabla \bar{\theta}_{n} \rightarrow \mathbb{K}(z, \theta) \nabla \theta \quad \text { in } L^{1+\delta}\left((0, T) \times \Omega ; \mathbb{R}^{d}\right),
$$

which, combined with convergences $(4.21)$ for $\bar{\eta}_{n}$, is enough to pass to the limit in the last term on the left-hand side of $(3.27 \mathrm{e})$.

Combining (4.1b), (4.1m), and (4.21) yields $\int_{0}^{\bar{\tau}_{n}(t)} \int_{\Omega} \bar{\theta}_{n} \mathbb{B}: e\left(\dot{u}_{n}\right) \bar{\eta}_{n} \mathrm{~d} x \mathrm{~d} s \rightarrow \int_{0}^{t} \int_{\Omega} \theta \mathbb{B}:$ $e(\dot{u}) \eta \mathrm{d} x \mathrm{~d} s$ as $n \rightarrow \infty$, while the passage to the limit in the term

$$
\int_{0}^{\bar{\tau}_{n}(t)} \int_{\Omega} \mathbb{D}\left(\underline{z}_{n}, \underline{\theta}_{n}\right) e\left(\dot{u}_{n}\right): e\left(\dot{u}_{n}\right) \bar{\eta}_{n} \mathrm{~d} x \mathrm{~d} s
$$

results from (4.20) combined with (4.21). Convergence (4.10) allows us to deal with the second term on the right-hand side of (3.27e), and we handle the last two terms via (3.24d) and (4.21), again. This concludes the proof of the weak heat equation and of the main existence result Theorem 2.6. 


\section{Asymptotic Behavior in the Slow Loading Regime: The Vanishing Viscosity and Inertia Limit}

In this section we address the limiting behavior of system (1.1) as the rate of the external load and of the heat sources becomes slower and slower. Accordingly, we will rescale time by a factor $\varepsilon>0$. For analytical reasons we restrict to the case of a Dirichlet problem in the displacement, namely within this section we shall suppose that

$$
\partial_{\mathrm{D}} \Omega=\partial \Omega .
$$

Like in the previous sections, we assume that the Dirichlet datum is homogeneous, cf. (1.3b).

As $\varepsilon \downarrow 0$ we will simultaneously pass to

1. a rate-independent system for the limit displacement and damage variables $(u, z)$, which does not display any temperature dependence and which formally reads

$$
\begin{array}{lr}
-\operatorname{div} \mathbb{C}(z) e(u)=f_{\mathrm{V}} & \text { in }(0, T) \times \Omega, \\
\partial \mathrm{R}_{1}(\dot{z})+\mathrm{D}_{z} G(z, \nabla z)-\operatorname{div}\left(\mathrm{D}_{\xi} G(z, \nabla z)\right)+\frac{1}{2} \mathbb{C}^{\prime}(z) e(u): e(u) \ni 0 & \text { in }(0, T) \times \Omega
\end{array}
$$

and will be weakly formulated through the concept of local solution to a rate-independent system;

2. a limit temperature $\theta=\Theta$, which is constant in space, but still time-dependent. The limit passage in the heat equation amounts to the trivial limit $0=0$, once more emphasizing that the limit system does not depend on temperature any more. A rescaling of the heat equation at level $\varepsilon$, however, reveals that $\Theta$ evolves in time according to an ODE in the sense of measures and the evolution is driven by the rate-independent dissipation and a measure originating from the viscous dissipation.

Indeed, for the limit system we expect that, if a change of heat is caused at some spot in the material, then the heat must be conducted all over the material with infinite speed, so that the temperature is kept constant in space. This justifies a scaling of the tensor of heat conduction coefficients for the systems at level $\varepsilon$. More precisely, we will suppose that

$$
\mathbb{K}_{\varepsilon}(z, \theta):=\frac{1}{\varepsilon^{\beta}} \mathbb{K}(z, \theta) \text { with } \mathbb{K} \text { satisfying (2.6) and } \beta>0 .
$$

While Proposition 5.2 holds with $\beta>0$, in Theorem 5.3 we shall require $\beta \geq 2$.

\subsection{Time Rescaling}

Let us now set up the vanishing viscosity analysis following [56], where this analysis was carried out for isothermal rate-independent processes in viscous solids, see also [15] in the context of perfect plasticity and $[58,66]$ for delamination, still in the isothermal case. We consider a family $\left(f_{\mathrm{V}, \varepsilon}, H_{\varepsilon}, h_{\varepsilon}\right)_{\varepsilon}$ of data for system (1.1) and we rescale $f_{\mathrm{V}, \varepsilon}, H_{\varepsilon}, h_{\varepsilon}$ by the factor $\varepsilon>0$, hence we introduce

$$
f^{\varepsilon}(t):=f_{\mathrm{V}, \varepsilon}(\varepsilon t) \quad H^{\varepsilon}(t):=H_{\varepsilon}(\varepsilon t), \quad h^{\varepsilon}(t):=h_{\varepsilon}(\varepsilon t) \quad \text { for } t \in\left[0, \frac{T}{\varepsilon}\right] .
$$

Theorem 2.6 guarantees that for every $\varepsilon>0$ there exists an energetic solution $\left(u^{\varepsilon}, z^{\varepsilon}, \theta^{\varepsilon}\right)$, defined on $\left[0, \frac{T}{\varepsilon}\right]$, to (the Cauchy problem for) system (1.1) supplemented with the data $f^{\varepsilon}, H^{\varepsilon}, h^{\varepsilon}$, and with the matrix of heat conduction coefficients $\mathbb{K}_{\varepsilon}$ from (5.2). For later convenience, let us recall that such solutions arise as limits of the time-discrete solutions to Problem 3.1. We now perform a rescaling of the solutions in such a way as to have them 
defined on the interval $[0, T]$. Namely, we set

$$
u_{\varepsilon}(t):=u^{\varepsilon}\left(\frac{t}{\varepsilon}\right), \quad z_{\varepsilon}(t):=z^{\varepsilon}\left(\frac{t}{\varepsilon}\right), \quad \theta_{\varepsilon}(t):=\theta^{\varepsilon}\left(\frac{t}{\varepsilon}\right) \quad \text { for } t \in[0, T] .
$$

It is not difficult to check that, after transforming the time scale, the triple $\left(u_{\varepsilon}, z_{\varepsilon}, \theta_{\varepsilon}\right)$ (formally) solves the following system in $(0, T) \times \Omega$ :

$$
\begin{aligned}
& \varepsilon^{2} \rho \ddot{u}_{\varepsilon}-\operatorname{div}\left(\varepsilon \mathbb{D}\left(z_{\varepsilon}, \theta_{\varepsilon}\right) e\left(\dot{u}_{\varepsilon}\right)+\mathbb{C}\left(z_{\varepsilon}\right) e\left(u_{\varepsilon}\right)-\theta_{\varepsilon} \mathbb{B}\right)=f_{\varepsilon}, \\
& \partial \mathrm{R}_{1}\left(\dot{z}_{\varepsilon}\right)+\mathrm{D}_{z} G\left(z_{\varepsilon}, \nabla z_{\varepsilon}\right)-\operatorname{div}\left(\mathrm{D}_{\xi} G\left(z_{\varepsilon}, \nabla z_{\varepsilon}\right)\right)+\frac{1}{2} \mathbb{C}^{\prime}\left(z_{\varepsilon}\right) e\left(u_{\varepsilon}\right): e\left(u_{\varepsilon}\right) \ni 0, \\
& \varepsilon \dot{\theta}_{\varepsilon}-\frac{1}{\varepsilon^{\beta}} \operatorname{div}\left(\mathbb{K}\left(z_{\varepsilon}, \theta_{\varepsilon}\right) \nabla \theta_{\varepsilon}\right)=\varepsilon \mathrm{R}_{1}\left(\dot{z}_{\varepsilon}\right)+\varepsilon^{2} \mathbb{D}\left(z_{\varepsilon}, \theta_{\varepsilon}\right) e\left(\dot{u}_{\varepsilon}\right): e\left(\dot{u}_{\varepsilon}\right) \\
& -\varepsilon \theta_{\varepsilon} \mathbb{B}: e\left(\dot{u}_{\varepsilon}\right)+H_{\varepsilon},
\end{aligned}
$$

with the original data $f_{\varepsilon}:=f_{\mathrm{V}, \varepsilon}, H_{\varepsilon}$, and $h_{\varepsilon}$, and complemented with the boundary conditions (1.3). Since in the following we will be interested in the limit of (5.3) as $\varepsilon \downarrow 0$, for notational simplicity we shall henceforth set $\rho=1$ in (5.3a).

Energetic solutions for the rescaled system (5.4)-(5.9). For later reference in the limit passage procedure as $\varepsilon \downarrow 0$, we recall the defining properties of energetic solutions. Given a quadruple of initial data $\left(u_{\varepsilon}^{0}, \dot{u}_{\varepsilon}^{0}, z_{\varepsilon}^{0}, \theta_{\varepsilon}^{0}\right)$ satisfying (2.7), a triple $\left(u_{\varepsilon}, z_{\varepsilon}, \theta_{\varepsilon}\right)$ is an energetic solution of the Cauchy problem for the PDE system (5.3) if it has the regularity (2.11), it complies with the initial conditions

$$
u_{\varepsilon}(0)=u_{\varepsilon}^{0}, \quad \dot{u}_{\varepsilon}(0)=\dot{u}_{\varepsilon}^{0}, \quad z_{\varepsilon}(0)=z_{\varepsilon}^{0}, \quad \theta_{\varepsilon}(0)=\theta_{\varepsilon}^{0} \quad \text { a.e. in } \Omega,
$$

and fulfills

- semistability and unidirectionality: for a.a. $x \in \Omega, z_{\varepsilon}(\cdot, x):[0, T] \rightarrow[0,1]$ is nonincreasing and for all $t \in[0, T]$

$$
\forall \tilde{z} \in \mathcal{Z}, \tilde{z} \leq z_{\varepsilon}(t): \quad \mathcal{E}_{\varepsilon}\left(t, u_{\varepsilon}(t), z_{\varepsilon}(t)\right) \leq \mathcal{E}_{\varepsilon}\left(t, u_{\varepsilon}(t), \tilde{z}\right)+\mathcal{R}_{1}\left(z_{\varepsilon}(t)-\tilde{z}\right),
$$

with the mechanical energy

$$
\mathcal{E}_{\varepsilon}(t, u, z):=\int_{\Omega}\left(\frac{1}{2} \mathbb{C}(z) e(u): e(u)+G(z, \nabla z)\right) \mathrm{d} x-\left\langle f_{\varepsilon}(t), u\right\rangle_{H_{\mathrm{D}}^{1}\left(\Omega ; \mathbb{R}^{d}\right)} ;
$$

- weak formulation of the momentum equation: for all test functions $v \in L^{2}\left(0, T ; H_{\mathrm{D}}^{1}\right.$ $\left.\left(\Omega ; \mathbb{R}^{d}\right)\right) \cap W^{1,1}\left(0, T ; L^{2}\left(\Omega ; \mathbb{R}^{d}\right)\right)$ and for all $t \in[0, T]$

$$
\begin{aligned}
& \varepsilon^{2} \int_{\Omega} \dot{u}_{\varepsilon}(t) \cdot v(t) \mathrm{d} x-\varepsilon^{2} \int_{0}^{t} \int_{\Omega} \dot{u}_{\varepsilon} \cdot \dot{v} \mathrm{~d} x \mathrm{~d} t \\
& \quad+\int_{0}^{t} \int_{\Omega}\left(\varepsilon \mathbb{D}\left(z_{\varepsilon}, \theta_{\varepsilon}\right) e\left(\dot{u}_{\varepsilon}\right)+\mathbb{C}\left(z_{\varepsilon}\right) e\left(u_{\varepsilon}\right)-\theta_{\varepsilon} \mathbb{B}\right): e(v) \mathrm{d} x \mathrm{~d} s \\
& \quad=\varepsilon^{2} \int_{\Omega} \dot{u}_{\varepsilon}^{0} \cdot v(0) \mathrm{d} x+\int_{0}^{t}\left\langle f_{\varepsilon}, v\right\rangle_{H_{\mathrm{D}}^{1}\left(\Omega ; \mathbb{R}^{d}\right)} \mathrm{d} s ;
\end{aligned}
$$

- mechanical energy equality: for all $t \in[0, T]$

$$
\begin{aligned}
& \frac{\varepsilon^{2}}{2} \int_{\Omega}\left|\dot{u}_{\varepsilon}(t)\right|^{2} \mathrm{~d} x+\varepsilon_{\varepsilon}\left(t, u_{\varepsilon}(t), z_{\varepsilon}(t)\right)+\int_{\Omega}\left(z_{\varepsilon}^{0}-z_{\varepsilon}(t)\right) \mathrm{d} x \\
& \quad+\int_{0}^{t} \int_{\Omega}\left(\varepsilon \mathbb{D}\left(z_{\varepsilon}, \theta_{\varepsilon}\right) e\left(\dot{u}_{\varepsilon}\right)-\theta_{\varepsilon} \mathbb{B}\right): e\left(\dot{u}_{\varepsilon}\right) \mathrm{d} x \mathrm{~d} s \\
& \quad=\frac{\varepsilon^{2}}{2} \int_{\Omega}\left|\dot{u}_{\varepsilon}^{0}\right|^{2} \mathrm{~d} x+\varepsilon_{\varepsilon}\left(0, u_{\varepsilon}^{0}, z_{\varepsilon}^{0}\right)+\int_{0}^{t} \partial_{t} \varepsilon_{\varepsilon}(s, u(s), z(s)) \mathrm{d} s ;
\end{aligned}
$$


- weak formulation of the heat equation: for all $t \in[0, T]$

$$
\begin{aligned}
& \varepsilon\left\langle\theta_{\varepsilon}(t), \eta(t)\right\rangle_{W^{2, d}+\delta}-\varepsilon \int_{0}^{t} \int_{\Omega} \theta_{\varepsilon} \dot{\eta} \mathrm{d} x \mathrm{~d} s+\frac{1}{\varepsilon^{\beta}} \int_{0}^{t} \int_{\Omega} \mathbb{K}\left(\theta_{\varepsilon}, z_{\varepsilon}\right) \nabla \theta_{\varepsilon} \cdot \nabla \eta \mathrm{d} x \mathrm{~d} s \\
& =\varepsilon \int_{\Omega} \theta_{\varepsilon}^{0} \eta(0) \mathrm{d} x+\int_{0}^{t} \int_{\Omega}\left(\varepsilon^{2} \mathbb{D}\left(z_{\varepsilon}, \theta_{\varepsilon}\right) e\left(\dot{u}_{\varepsilon}\right): e\left(\dot{u}_{\varepsilon}\right)-\varepsilon \theta_{\varepsilon} \mathbb{B}: e\left(\dot{u}_{\varepsilon}\right)\right) \eta \mathrm{d} x \mathrm{~d} s \\
& \quad+\varepsilon \int_{0}^{t} \int_{\Omega} \eta\left|\dot{z}_{\varepsilon}\right| \mathrm{d} x \mathrm{~d} s+\int_{0}^{t} \int_{\partial \Omega} h_{\varepsilon} \eta \mathrm{d} \mathcal{H}^{d-1}(x) \mathrm{d} s+\int_{0}^{t} \int_{\Omega} H_{\varepsilon} \eta \mathrm{d} x \mathrm{~d} s
\end{aligned}
$$

for all test functions $\eta \in H^{1}\left(0, T ; L^{2}(\Omega)\right) \cap C^{0}\left(0, T ; W^{2, d+\delta}(\Omega)\right)$ (recall that $\left|\dot{z}_{\varepsilon}\right|$ denotes the total variation measure of $z_{\varepsilon}$ ).

Remark 5.1 Let us also observe that testing (5.9) by $\frac{1}{\varepsilon}$ and summing up with (5.8) leads to the rescaled total energy equality

$$
\begin{aligned}
& \frac{\varepsilon^{2}}{2} \int_{\Omega}\left|\dot{u}_{\varepsilon}(t)\right|^{2} \mathrm{~d} x+\mathcal{E}_{\varepsilon}\left(t, u_{\varepsilon}(t), z_{\varepsilon}(t)\right)+\int_{\Omega} \theta_{\varepsilon}(t) \mathrm{d} x \\
& =\frac{\varepsilon^{2}}{2} \int_{\Omega}\left|\dot{u}_{\varepsilon}^{0}\right|^{2} \mathrm{~d} x+\mathcal{E}_{\varepsilon}\left(0, u_{\varepsilon}^{0}, z_{\varepsilon}^{0}\right)+\int_{\Omega} \theta_{\varepsilon}^{0} \mathrm{~d} x \\
& \quad+\int_{0}^{t} \partial_{t} \mathcal{E}_{\varepsilon}\left(s, u_{\varepsilon}(s), z_{\varepsilon}(s)\right) \mathrm{d} s+\frac{1}{\varepsilon} \int_{0}^{t} \int_{\partial \Omega} h_{\varepsilon} \mathrm{d} \mathcal{H}^{d-1}(x) \mathrm{d} s+\frac{1}{\varepsilon} \int_{0}^{t} \int_{\Omega} H_{\varepsilon} \mathrm{d} x \mathrm{~d} s .
\end{aligned}
$$

\subsection{A Priori Estimates Uniform with Respect to $\varepsilon$}

As done in the proof of Theorem 2.6, we shall derive the basic a priori estimates on the rescaled solutions $\left(u_{\varepsilon}, z_{\varepsilon}, \theta_{\varepsilon}\right)_{\varepsilon}$ from the total energy equality (5.10). Therefore, it is clear that we shall have to assume that the families of data $\left(H_{\varepsilon}\right)_{\varepsilon}$ and $\left(h_{\varepsilon}\right)_{\varepsilon}$ converge to zero in the sense that there exists $C>0$ such that for all $\varepsilon>0$

$$
\int_{0}^{t} \int_{\Omega} H_{\varepsilon} \mathrm{d} x \mathrm{~d} s \leq C \varepsilon, \quad \int_{0}^{t} \int_{\partial \Omega} h_{\varepsilon} \mathrm{d} \mathcal{H}^{d-1}(x) \mathrm{d} s \leq C \varepsilon .
$$

Furthermore, we shall suppose that there exists $f$ such that

$$
f_{\varepsilon} \rightarrow f \quad \text { in } H^{1}\left(0, T ; H_{\mathrm{D}}^{1}\left(\Omega ; \mathbb{R}^{d}\right)^{*}\right) .
$$

We are now in a position to derive a priori bounds on the rescaled solutions $\left(u_{\varepsilon}, z_{\varepsilon}, \theta_{\varepsilon}\right)_{\varepsilon}$, uniform with respect to $\varepsilon>0$. These estimates are the time-continuous counterpart of the First-Third a priori estimates in the proof of Proposition 3.4. Actually, the calculations underlying the Second and Third estimates can be performed only formally, when arguing on the energetic formulation of system (5.3). Indeed, these computations are based on testing the weak heat equation (5.9) by $\theta_{\varepsilon}^{\alpha-1}$, which is not admissible since $\theta_{\varepsilon}^{\alpha-1} \notin \mathrm{C}^{0}\left([0, T] ; W^{2, d+\delta}(\Omega)\right)$.

That is why Proposition 5.2 below will be stated not for all energetic solutions to the rescaled system (5.3), but just for those arising from the discrete solutions to (5.3) constructed in Sect. 3.1. More precisely, we shall call "approximable solution" to the rescaled system (5.3) any triple obtained in the time-discrete to continuous limit, for which convergences (4.1) of Proposition 4.1 hold; in Sect. 4 we have shown that any approximable solution is an energetic solution. Now, it can be checked that some of the a priori estimates on the discrete solutions in Proposition 3.4 (i.e. those corresponding to (5.14) below) are uniform 
with respect to $\tau$ and $\varepsilon$ as well. Therefore, Proposition 4.1 ensures that they are inherited by the "approximable" solutions in the limit $\tau \downarrow 0$, still uniformly with respect to $\varepsilon$.

Nonetheless, to simplify the exposition, in the proof of Proposition 5.2 we will no longer work on the time-discrete scheme but rather develop the calculations directly (and sometimes only formally) on the time-continuous level.

Proposition 5.2 (A priori estimates) Assume (2.1)-(2.5), (5.2) with $\beta>0,\left(H_{\varepsilon}\right)_{\varepsilon} \subset$ $L^{1}\left(0, T ; L^{1}(\Omega)\right) \cap L^{2}\left(0, T ; H^{1}(\Omega)^{*}\right),\left(h_{\varepsilon}\right)_{\varepsilon} \subset L^{1}\left(0, T ; L^{2}(\partial \Omega)\right)$ fulfill (5.11), and $\left(f_{\varepsilon}\right)_{\varepsilon} \subset$ $H^{1}\left(0, T ; H_{\mathrm{D}}^{1}\left(\Omega ; \mathbb{R}^{d}\right)^{*}\right)$ comply with (5.12). In addition to (2.7), let the family of initial data $\left(u_{\varepsilon}^{0}, \dot{u}_{\varepsilon}^{0}, z_{\varepsilon}^{0}, \theta_{\varepsilon}^{0}\right)_{\varepsilon}$ fulfill

$$
\left|\mathcal{E}_{\varepsilon}\left(0, u_{\varepsilon}^{0}, z_{\varepsilon}^{0}\right)\right|+\varepsilon\left\|\dot{u}_{\varepsilon}^{0}\right\|_{L^{2}\left(\Omega ; \mathbb{R}^{d}\right)}+\left\|\theta_{\varepsilon}^{0}\right\|_{L^{1}(\Omega)} \leq C
$$

for a constant $C$ independent of $\varepsilon$. Let $\left(u_{\varepsilon}, z_{\varepsilon}, \theta_{\varepsilon}\right)_{\varepsilon}$ be a family of approximable solutions to system (5.3). Then, there exists a constant $C>0$ such that the following estimates hold for all $\varepsilon>0$ :

$$
\begin{aligned}
\left\|u_{\varepsilon}\right\|_{L^{\infty}\left(0, T ; H_{\mathrm{D}}^{1}\left(\Omega ; \mathbb{R}^{d}\right)\right)} & \leq C, \\
\varepsilon\left\|\dot{u}_{\varepsilon}\right\|_{L^{\infty}\left(0, T ; L^{2}\left(\Omega ; \mathbb{R}^{d}\right)\right)} & \leq C, \\
\mathcal{R}_{1}\left(z_{\varepsilon}(T)-z_{\varepsilon}^{0}\right) & \leq C, \\
\left\|z_{\varepsilon}\right\|_{L^{\infty}((0, T) \times \Omega)} & \leq 1, \\
\left\|z_{\varepsilon}\right\|_{L^{\infty}\left(0, T ; W^{1, q}(\Omega)\right)} & \leq C, \\
\left\|\theta_{\varepsilon}\right\|_{L^{\infty}\left(0, T ; L^{1}(\Omega)\right)} & \leq C, \\
\left\|\nabla \theta_{\varepsilon}\right\|_{L^{2}\left(0, T ; L^{2}\left(\Omega ; \mathbb{R}^{d}\right)\right)} & \leq C \varepsilon^{\beta / 2}, \\
\left\|\theta_{\varepsilon}\right\|_{L^{2}\left(0, T ; H^{1}(\Omega)\right)} & \leq C, \\
\left\|\theta_{\varepsilon}\right\|_{L^{p}((0, T) \times \Omega)} & \leq C \text { for any } p \in\left\{\begin{array}{l}
{[1,8 / 3] \text { if } d=3,} \\
{[1,3] \quad \text { if } d=2,}
\end{array}\right.
\end{aligned}
$$

with $\mathcal{R}_{1}$ from (1.2).

Sketch of the proof First a priori estimate: ad (5.14a), (5.14b), (5.14c), (5.14d), (5.14e), (5.14f): Estimate (5.14d) is obvious. Estimate (5.14c) follows from the definition of $\mathcal{R}_{1}$, (2.5a), and (2.7a), and the fact that the functions $z_{\varepsilon}(\cdot, x)$ are nonincreasing. We start from the total energy equality (5.10). Also thanks to (5.12), the energies $\mathcal{E}_{\varepsilon}$ enjoy the coercivity property (3.33) with constants independent of $\varepsilon$. Therefore, relying on the uniform bound (5.12) for $\dot{f}_{\varepsilon}$, and using that $\theta_{\varepsilon}>0$ a.e. in $(0, T) \times \Omega$ for every $\varepsilon>0$, one can repeat the very same calculations as in the first step of the proof of Proposition 3.4, and conclude that the left-hand side of (5.10) is uniformly bounded from above and from below, whence (5.14a), (5.14b), (5.14e), (5.14f).

Second and third a priori estimates: ad (5.14g), (5.14h), and (5.14i): We (formally) test (5.9) by $\theta_{\varepsilon}^{\alpha-1}$, integrate in time, and arrive at the (formally written) analogue of (3.35), viz. 


$$
\begin{aligned}
& \frac{c}{\varepsilon^{\beta}} \int_{0}^{t} \int_{\Omega} \mathbb{K}\left(z_{\varepsilon}, \theta_{\varepsilon}\right) \nabla\left(\theta_{\varepsilon}^{\alpha / 2}\right) \cdot \nabla\left(\theta_{\varepsilon}^{\alpha / 2}\right) \mathrm{d} x \mathrm{~d} s+\varepsilon^{2} \int_{0}^{t} \int_{\Omega} \mathbb{D}\left(z_{\varepsilon}, \theta_{\varepsilon}\right) e\left(\dot{u}_{\varepsilon}\right): e\left(\dot{u}_{\varepsilon}\right) \theta_{\varepsilon}^{\alpha-1} \mathrm{~d} x \mathrm{~d} s \\
& \quad+\varepsilon \int_{0}^{t} \int_{\Omega} \theta_{\varepsilon}^{\alpha-1}\left|\dot{z}_{\varepsilon}\right| \mathrm{d} x \mathrm{~d} s+\int_{0}^{t} \int_{\partial \Omega} h_{\varepsilon} \theta_{\varepsilon}^{\alpha-1} \mathrm{~d} \mathcal{H}^{d-1}(x) \mathrm{d} s+\int_{0}^{t} \int_{\Omega} H_{\varepsilon} \theta_{\varepsilon}^{\alpha-1} \mathrm{~d} x \mathrm{~d} s \\
& =\varepsilon \int_{0}^{t} \int_{\Omega} \dot{\theta}_{\varepsilon} \theta_{\varepsilon}^{\alpha-1} \mathrm{~d} x \mathrm{~d} s+\varepsilon \int_{0}^{t} \int_{\Omega} \theta_{\varepsilon} \mathbb{B}: e\left(\dot{u}_{\varepsilon}\right) \theta_{\varepsilon}^{\alpha-1} \mathrm{~d} x \mathrm{~d} s \doteq I_{1}+I_{2} .
\end{aligned}
$$

As in the proof of Proposition 3.4, we estimate

$$
I_{1}=\varepsilon \int_{\Omega} \frac{\left(\theta_{\varepsilon}(t)\right)^{\alpha}}{\alpha} \mathrm{d} x-\varepsilon \int_{\Omega} \frac{\left(\theta_{\varepsilon}^{0}\right)^{\alpha}}{\alpha} \mathrm{d} x,
$$

whereas we estimate $I_{2}=\iint \varepsilon \theta_{\varepsilon} \mathbb{B}: e\left(\dot{u}_{\varepsilon}\right) \theta_{\varepsilon}^{\alpha-1}$ by

$$
I_{2} \leq \varepsilon^{2} \frac{C_{\mathbb{D}}^{1}}{2} \int_{0}^{t} \int_{\Omega}\left|e\left(\dot{u}_{\varepsilon}\right)\right|^{2} \theta_{\varepsilon}^{\alpha-1} \mathrm{~d} x \mathrm{~d} s+C \int_{0}^{t} \int_{\Omega}\left|\theta_{\varepsilon}\right|^{2} \theta_{\varepsilon}^{\alpha-1} \mathrm{~d} x \mathrm{~d} s,
$$

where the constant $C$ subsumes the norm $|\mathbb{B}|$ as well. Combining (5.15)-(5.17) and then arguing exactly in the same way as in the proof of Proposition 3.4, we end up with the analogue of (3.36), i.e.,

$$
\begin{aligned}
& \frac{1}{\varepsilon^{\beta}} \int_{0}^{t} \int_{\Omega} \mathbb{K}\left(z_{\varepsilon}, \theta_{\varepsilon}\right) \nabla\left(\theta_{\varepsilon}^{\alpha / 2}\right) \cdot \nabla\left(\theta_{\varepsilon}^{\alpha / 2}\right) \mathrm{d} x \mathrm{~d} s+\int_{\Omega} \frac{\varepsilon}{\alpha}\left(\theta_{\varepsilon}^{0}\right)^{\alpha} \mathrm{d} x \leq \int_{\Omega} \frac{\varepsilon}{\alpha}\left(\theta_{\varepsilon}(t)\right)^{\alpha} \mathrm{d} x \\
& \quad+C \int_{0}^{t} \int_{\Omega} \theta_{\varepsilon}^{\alpha+1}(s) \mathrm{d} x \mathrm{~d} s,
\end{aligned}
$$

whence $\frac{1}{\varepsilon^{\beta}} \int_{0}^{T} \int_{\Omega} \mathbb{K}\left(z_{\varepsilon}, \theta_{\varepsilon}\right) \nabla\left(\theta_{\varepsilon}^{\alpha / 2}\right) \cdot \nabla\left(\theta_{\varepsilon}^{\alpha / 2}\right) \mathrm{d} x \mathrm{~d} t \leq C$. From this, with the same arguments as in the third step of the proof of Proposition 3.4, cf. (3.41), we infer that

$$
\int_{0}^{T} \int_{\Omega}\left|\nabla \theta_{\varepsilon}\right|^{2} \mathrm{~d} x \mathrm{~d} t \leq C \varepsilon^{\beta}
$$

i.e. $(5.14 \mathrm{~g})$. Then, $(5.14 \mathrm{~h})$ follows from $(5.14 \mathrm{~g})$ and $(5.14 \mathrm{f})$, via the Poincaré inequality. Finally, (5.14i) ensues by interpolation, as in the proof of Proposition 3.4.

Observe that in the proof of Proposition 5.2 we have not been able to repeat the calculations in the Fourth and Fifth estimates, cf. the proof of Proposition 3.4. In particular, from the mechanical energy equality (5.8) we have not been able to deduce an estimate for $\varepsilon^{1 / 2} e\left(\dot{u}_{\varepsilon}\right)$ in $L^{2}\left(0, T ; L^{2}\left(\Omega ; \mathbb{R}^{d \times d}\right)\right)$, since we cannot bound the term $\int_{0}^{t} \int_{\Omega} \theta_{\varepsilon}: e\left(\dot{u}_{\varepsilon}\right) \mathrm{d} x \mathrm{~d} s$ on the right-hand side of (5.8). Therefore, in the proof of our convergence result for vanishing viscosity and inertia, Theorem 5.3 below, we shall have to resort to careful arguments in order to handle the terms containing $e\left(\dot{u}_{\varepsilon}\right)$, in the passage to the limit in the momentum equation and mechanical energy equality, cf. (5.30)-(5.33). In particular, differently from Proposition 3.4, for a vanishing sequence $\left(\varepsilon_{n}\right)_{n}$ the convergences

$\varepsilon_{n} e\left(\dot{u}_{\varepsilon_{n}}\right) \rightarrow 0$ strongly in $L^{2}\left(0, T ; L^{2}\left(\Omega ; \mathbb{R}^{d \times d}\right)\right)$ and $\int_{0}^{t} \int_{\Omega} \theta_{\varepsilon_{n}}: e\left(\dot{u}_{\varepsilon_{n}}\right) \mathrm{d} x \mathrm{~d} s \rightarrow 0$, $\theta_{\varepsilon} \rightarrow \Theta$ strongly in $\left.L^{2}(0, T) \times \Omega\right)$

will now be extracted from the weak heat equation (5.9), using integration by parts and the information that $\Theta$ is constant in space. It is in this connection that we need to further assume homogeneous Dirichlet boundary conditions for the displacement on the whole boundary $\partial \Omega$, cf. (5.1).

\section{를 Springer}




\subsection{Convergence to Local Solutions of the Rate-Independent Limit System}

Let us mention in advance that in Theorem 5.3 we will prove that, up to a subsequence, the functions $\left(u_{\varepsilon}, z_{\varepsilon}, \theta_{\varepsilon}\right)$ converge to a limit triple $(u, z, \Theta)$ such that $\Theta$ is spatially constant. As we will see, the pair $(u, z)$ fulfills the (pointwise-in-time) static momentum balance (i.e. without viscosity and inertia), a semistability condition with respect to the energy $\mathcal{E}$ arising from $\mathcal{E}_{\varepsilon}$ (5.6) in the limit $\varepsilon \downarrow 0$, and an energy inequality, where the viscous, the inertial, and the thermal expansion contributions are no longer present. This inequality holds on $[0, t]$ for every $t \in[0, T]$ in the general case, and on $[s, t]$ for all $t \in[0, T]$ and almost every $s \in(0, t)$, under a further condition on the gradient term in the energy $\mathcal{E}$, i.e. that $q>d$. Indeed, the three properties (momentum balance, semistability, energy inequality) constitute the notion of local solution $[41,58,65]$ to the rate-independent system driven by $\mathcal{R}_{1}$ and $\mathcal{E}$. Observe that, in fact, the spatially constant $\Theta$ does not appear in these relations, because it contributes with a zero term to the momentum balance.

Moreover, testing the weak heat equation (5.9) with functions $\eta$ that are constant in space (which is the property of the limit temperature $\Theta$ by $(5.14 \mathrm{~g})$ ) and taking into account the bounds (5.11), (5.13), (5.14f), and convergence (5.18), we find in the limit relation $0=0$. This shows that the temporal evolution of $\Theta$ is irrelevant in the rate-independent limit model. In fact, in order to gain insight into the time evolution of $\Theta$, we will perform the limit passage in the heat equation (5.9) rescaled by the factor $1 / \varepsilon$ and tested by $\eta \in H^{1}(0, T)$, constant in space. In this way, the heat-transfer term involving $\mathbb{K}_{\varepsilon}=\frac{1}{\varepsilon \beta} \mathbb{K}$ will disappear. This will lead to an ODE for the limit function $\Theta$, cf. (5.26). Such an ODE involves a defect measure $\mu$, i.e. a Radon measure on $[0, T]$ arising in the limit of the viscous dissipation term $\left\|\varepsilon \mathbb{D}\left(z_{\varepsilon}, \theta_{\varepsilon}\right) e\left(\dot{u}_{\varepsilon}\right): e\left(\dot{u}_{\varepsilon}\right)\right\|_{L^{1}(\Omega)}$, see (5.27) below.

In the following proof, notice that Steps $0-3$ can be proven for $\beta>0$, while in Step 4 we need $\beta \geq 2$. Furthermore, the condition that the tensor $\mathbb{B}$ is constant in space will have a crucial role in handling the thermal expansion term $\theta_{\varepsilon} \mathbb{B}: e\left(\dot{u}_{\varepsilon}\right)$ in the rescaled heat equation, cf. (5.32) ahead.

Theorem 5.3 Assume (2.1)-(2.4), (2.5), (2.8), and, in addition, let (5.1), (5.2) with $\beta \geq 2$, (5.11), and (5.12) be satisfied. Let the initial data $\left(u_{\varepsilon}^{0}, \dot{u}_{\varepsilon}^{0}, z_{\varepsilon}^{0}, \theta_{\varepsilon}^{0}\right)_{\varepsilon}$ fulfill (2.7), (5.13),

$$
\varepsilon \dot{u}_{\varepsilon}^{0} \rightarrow 0 \text { in } L^{2}\left(\Omega ; \mathbb{R}^{d}\right),
$$

and suppose that there exist $u_{0} \in H_{\mathrm{D}}^{1}\left(\Omega ; \mathbb{R}^{d}\right)$ and $z_{0} \in Z$ such that

$$
u_{\varepsilon}^{0} \rightarrow u_{0} \text { in } H_{\mathrm{D}}^{1}\left(\Omega ; \mathbb{R}^{d}\right), \quad z_{\varepsilon}^{0} \rightarrow z_{0} \text { in } z, \quad \mathcal{E}_{\varepsilon}\left(0, u_{\varepsilon}^{0}, z_{\varepsilon}^{0}\right) \rightarrow \mathcal{E}\left(0, u_{0}, z_{0}\right) \quad \text { as } \varepsilon \downarrow 0,
$$

with $\varepsilon_{\varepsilon}$ as in (5.6).

Then, the functions $\left(u_{\varepsilon}, z_{\varepsilon}, \theta_{\varepsilon}\right)_{\varepsilon}$ converge (up to subsequences) to a triple $(u, z, \Theta)$ such that

$$
\begin{gathered}
u \in L^{\infty}\left(0, T ; H_{\mathrm{D}}^{1}\left(\Omega ; \mathbb{R}^{d}\right)\right), \quad z \in L^{\infty}\left(0, T ; W^{1, q}(\Omega)\right) \cap L^{\infty}((0, T) \times \Omega) \cap \mathrm{BV}\left([0, T] ; L^{1}(\Omega)\right), \\
\Theta \text { is constant in space and } \Theta \in L^{p}(0, T) \text { for any } p \in \begin{cases}{[1,8 / 3]} & \text { if } d=3, \\
{[1,3]} & \text { if } d=2 .\end{cases}
\end{gathered}
$$

The pair $(u, z)$ fulfills the unidirectionality as well as

1. the semistability condition (2.12a) for all $t \in[0, T]$, with the mechanical energy $\mathcal{E}$ defined as in (5.6) with $f_{\varepsilon}$ replaced by the weak limit $f$ of the sequence $\left(f_{\varepsilon}\right)_{\varepsilon}$, see (5.12); 
2. the weak momentum balance for all $t \in[0, T]$

$$
\int_{\Omega} \mathbb{C}(z(t)) e(u(t)): e(v) \mathrm{d} x=\langle f(t), v\rangle_{H_{\mathrm{D}}^{1}\left(\Omega ; \mathbb{R}^{d}\right)} \quad \text { for all } v \in H_{\mathrm{D}}^{1}\left(\Omega ; \mathbb{R}^{d}\right) ;
$$

3. the mechanical energy inequality for all $t \in[0, T]$

$$
\mathcal{E}(t, u(t), z(t))+\int_{\Omega}(z(0)-z(t)) \mathrm{d} x \leq \mathcal{E}(0, u(0), z(0))+\int_{0}^{t} \partial_{t} \mathcal{E}(r, u(r), z(r)) \mathrm{d} r ;
$$

If in addition the function $G$ fulfills the growth condition $(2.5 \mathrm{~d})$ with $q>d$, then $(u, z)$ also fulfill

$$
\mathcal{E}(t, u(t), z(t))+\int_{\Omega}(z(s)-z(t)) \mathrm{d} x \leq \mathcal{E}(s, u(s), z(s))+\int_{s}^{t} \partial_{t} \mathcal{E}(r, u(r), z(r)) \mathrm{d} r
$$

for all $t \in[0, T]$ and for almost all $s \in(0, t)$.

Moreover, assume in addition that there exists $\widetilde{H} \in L^{1}(0, T)$ such that

$$
\frac{1}{\varepsilon}\left(\left\|H_{\varepsilon}\right\|_{L^{1}(\Omega)}+\left\|h_{\varepsilon}\right\|_{L^{1}(\partial \Omega)}\right) \rightarrow \widetilde{H} \quad \text { in } L^{1}(0, T) .
$$

Then, $\Theta$ fulfills

$$
\begin{gathered}
\eta(t) \int_{\Omega} \Theta(t) \mathrm{d} x-\int_{0}^{t} \dot{\eta} \int_{\Omega} \Theta \mathrm{d} x \mathrm{~d} s-\eta(0) \int_{\Omega} \Theta(0) \mathrm{d} x \\
=\int_{0}^{t} \eta \mathrm{d} \mu(s)+\int_{0}^{t} \eta \int_{\Omega}|\dot{z}| \mathrm{d} x \mathrm{~d} s+\int_{0}^{t} \widetilde{H} \eta \mathrm{d} s
\end{gathered}
$$

for a.a. $t \in(0, T)$ and for every $\eta \in H^{1}(0, T)$ constant in space, with the defect measure $\mu$ given by

$$
\left\|\varepsilon \mathbb{D}\left(z_{\varepsilon}, \theta_{\varepsilon}\right) e\left(\dot{u}_{\varepsilon}\right): e\left(\dot{u}_{\varepsilon}\right)\right\|_{L^{1}(\Omega)} \rightarrow \mu \quad \text { in the sense of Radon measures in }[0, T] .
$$

Proof Step 0, compactness: It follows from Proposition 5.2 that for every vanishing sequence $\left(\varepsilon_{n}\right)_{n}$ there exist a (not relabeled) subsequence and a triple $(u, z, \Theta)$ as in (5.21) such that the following convergences hold

$$
\begin{aligned}
u_{\varepsilon_{n}} \stackrel{*}{\rightarrow} u & \text { in } L^{\infty}\left(0, T ; H_{\mathrm{D}}^{1}\left(\Omega ; \mathbb{R}^{d}\right)\right) \\
\varepsilon_{n} u_{\varepsilon_{n}} \stackrel{*}{\rightarrow} 0 & \text { in } W^{1, \infty}\left(0, T ; L^{2}\left(\Omega ; \mathbb{R}^{d}\right)\right) \\
z_{\varepsilon_{n}} \stackrel{*}{\rightarrow} z & \text { in } L^{\infty}\left(0, T ; W^{1, q}(\Omega)\right) \cap L^{\infty}((0, T) \times \Omega) \\
z_{\varepsilon_{n}}(t) \rightarrow z(t) & \text { in } W^{1, q}(\Omega) \text { for all } t \in[0, T] \\
z_{\varepsilon_{n}}(t) \rightarrow z(t) & \text { in } L^{r}(\Omega) \text { for all } 1 \leq r<\infty \text { and for all } t \in[0, T] \\
\theta_{\varepsilon_{n}} \rightarrow \Theta & \text { in } L^{2}\left(0, T ; H^{1}(\Omega)\right) \cap L^{p}((0, T) \times \Omega) \text { for all } p \text { as in }(5.14 i) .
\end{aligned}
$$

Indeed, (5.28a) ensues from (5.14a), and it gives, in particular, that $\varepsilon_{n} u_{\varepsilon_{n}} \rightarrow 0$ in $L^{\infty}\left(0, T ; H_{\mathrm{D}}^{1}\left(\Omega ; \mathbb{R}^{d}\right)\right)$. Then, convergence (5.28b) directly follows from estimate $(5.14 \mathrm{~b})$. Convergences $(5.28 \mathrm{c})-(5.28 \mathrm{e})$ ensue from the very same compactness arguments as in the proof of Proposition 4.1, also using the Helly Theorem. Furthermore, (5.28f) follows from estimates $(5.14 \mathrm{~h})-(5.14 \mathrm{i})$ by weak compactness. Observe that in view of $(5.14 \mathrm{~g})$ we have that

$$
\nabla \theta_{\varepsilon_{n}} \rightarrow 0 \quad \text { in } L^{2}\left(0, T ; L^{2}\left(\Omega ; \mathbb{R}^{d}\right)\right)
$$

\section{Springer}


Therefore, we conclude that $\nabla \Theta=0$ a.e. in $(0, T) \times \Omega$. Since $\Theta$ is spatially constant, hereafter we will write it as a function of the sole variable $t$.

We now prove the enhanced convergence

$$
\theta_{\varepsilon_{n}} \rightarrow \Theta \text { in } L^{2}\left(0, T ; L^{2}(\Omega)\right) .
$$

In fact, we use the Poincaré inequality

$$
\begin{aligned}
& \left\|\theta_{\varepsilon_{n}}-\Theta\right\|_{L^{2}\left(0, T ; L^{2}(\Omega)\right)} \\
& \leq\left\|\nabla\left(\theta_{\varepsilon_{n}}-\Theta\right)\right\|_{L^{2}\left(0, T ; L^{2}\left(\Omega ; \mathbb{R}^{d}\right)\right)} \\
& \quad+C(\Omega, T)\left|\int_{0}^{T} \int_{\Omega}\left(\theta_{\varepsilon_{n}}-\Theta\right) \mathrm{d} x \mathrm{~d} s\right| \longrightarrow 0
\end{aligned}
$$

where the gradient term tends to 0 by (5.29), and the convergence of the second term follows from (5.28f).

Finally, let us show that

$$
\varepsilon_{n} e\left(\dot{u}_{\varepsilon_{n}}\right) \rightarrow 0 \text { strongly in } L^{2}\left(0, T ; L^{2}\left(\Omega ; \mathbb{R}^{d \times d}\right)\right) .
$$

Preliminarily, observe that, since $\mathbb{B}$ and the limit function $\Theta$ are constant in space, we have by integration by parts

$$
\int_{0}^{t} \int_{\Omega} \Theta \mathbb{B}: e\left(\dot{u}_{\varepsilon_{n}}\right) \mathrm{d} x \mathrm{~d} s=\int_{0}^{t} \int_{\partial \Omega} \Theta \mathbb{B} v \cdot \dot{u}_{\varepsilon_{n}} \mathrm{~d} \mathcal{H}^{d-1}(x) \mathrm{d} s-\int_{0}^{t} \int_{\Omega} \operatorname{div}(\Theta \mathbb{B}) \cdot \dot{u}_{\varepsilon_{n}} \mathrm{~d} x \mathrm{~d} s=0,
$$

where we used $\partial_{\mathrm{D}} \Omega=\partial \Omega$, hence $\dot{u}_{\varepsilon_{n}} \in L^{2}\left(0, T ; H_{\mathrm{D}}^{1}\left(\Omega ; \mathbb{R}^{d}\right)\right)$ implies that $\dot{u}_{\varepsilon_{n}}=0$ a.e. in $(0, T) \times \partial \Omega$. Using (5.32) in the weak heat equation (5.9) tested by 1 and applying Young's inequality, we find

$$
\begin{aligned}
& \varepsilon_{n}\left(\int_{\Omega}\left(\theta_{\varepsilon_{n}}(t)-\theta_{\varepsilon_{n}}^{0}\right) \mathrm{d} x\right) \\
& \quad \geq \int_{0}^{t} \int_{\Omega}\left[\varepsilon_{n}^{2} \mathbb{D}\left(z_{\varepsilon_{n}}, \theta_{\varepsilon_{n}}\right) e\left(\dot{u}_{\varepsilon_{n}}\right): e\left(\dot{u}_{\varepsilon_{n}}\right)-\varepsilon_{n}\left(\theta_{\varepsilon_{n}}-\Theta \mathbb{B}\right): e\left(\dot{u}_{\varepsilon_{n}}\right)\right] \mathrm{d} x \mathrm{~d} s \\
& \quad \geq \int_{0}^{t} \int_{\Omega} \varepsilon_{n}^{2} \frac{C_{\mathbb{D}}}{2}\left|e\left(\dot{u}_{\varepsilon_{n}}\right)\right|^{2} \mathrm{~d} x \mathrm{~d} s-C\left\|\theta_{\varepsilon_{n}}-\Theta\right\|_{L^{2}\left(0, T ; L^{2}(\Omega)\right)}^{2}
\end{aligned}
$$

with $C=|\mathbb{B}| / 2$. From this, taking into account that $\left(\theta_{\varepsilon_{n}}^{0}\right)_{n}$ is bounded in $L^{1}(\Omega)$ by (5.13), estimate (5.14f) for $\left(\theta_{\varepsilon_{n}}\right)_{n}$, and convergence (5.30), we conclude that $\lim _{\varepsilon_{n} \downarrow 0} \varepsilon_{n} \| e\left(\dot{u}_{\varepsilon_{n}}\right)$ $\|_{L^{2}\left(0, T ; L^{2}\left(\Omega ; \mathbb{R}^{d \times d}\right)\right)}=0$, whence (5.31).

In fact, by Korn's inequality we conclude that

$$
\varepsilon_{n} u_{\varepsilon_{n}} \rightarrow 0 \text { in } H^{1}\left(0, T ; H_{\mathrm{D}}^{1}\left(\Omega ; \mathbb{R}^{d}\right)\right) .
$$

Step 1, passage to the limit in the momentum balance (5.7): Convergence (5.34), joint with the boundedness $(2.3 \mathrm{e})$ of the tensor $\mathbb{D}$, ensures that the first and the second summands on the left-hand side of (5.7) tend to zero. Arguing as in the proof of Proposition 4.3, we show that for every test function $v$ in $(5.7), \mathbb{C}\left(z_{\varepsilon_{n}}\right) e(v) \rightarrow \mathbb{C}(z) e(v)$ in $L^{2}\left((0, T) \times \Omega ; \mathbb{R}^{d \times d}\right)$. We combine this with (5.28a) and, also using (5.28f), we pass to the limit in the third term on the left-hand side of (5.7), recalling that the fourth summand converges to zero similarly to (5.32). As for the right-hand side, by (5.13) we have

$$
\varepsilon_{n}^{2} \dot{u}_{\varepsilon_{n}}^{0} \rightarrow 0 \quad \text { in } L^{2}\left(\Omega ; \mathbb{R}^{d}\right),
$$


hence the first term converges to zero. The second one tends to zero for almost all $t \in(0, T)$ by (5.28b), which in particular gives

$$
\varepsilon_{n}^{2} \dot{u}_{\varepsilon_{n}} \rightarrow 0 \quad \text { in } L^{\infty}\left(0, T ; L^{2}\left(\Omega ; \mathbb{R}^{d}\right)\right) .
$$

For the third one, we use (5.12). We thus conclude that (5.22) holds at almost all $t \in(0, T)$.

In order to check it at every $t \in[0, T]$, we observe that for every $t \in[0, T]$ from the bounded sequence $\left(u_{\varepsilon_{n}}(t)\right)_{n}$ (along which convergences (5.28) hold) we can extract a subsequence, possibly depending on $t$, weakly converging to some $\bar{u}(t)$ in $H_{\mathrm{D}}^{1}\left(\Omega ; \mathbb{R}^{d}\right)$. Relying on convergence (5.28e) for $\left(z_{\varepsilon_{n}}(t)\right)_{n}$ and on (5.12) for $\left(f_{\varepsilon_{n}}(t)\right)$, with the same arguments as above we conclude that $\int_{\Omega} \mathbb{C}(z(t)) e(\bar{u}(t)): e(v) \mathrm{d} x=\langle f(t), v\rangle_{H_{\mathrm{D}}^{1}\left(\Omega ; \mathbb{R}^{d}\right)}$ for all $v \in H_{\mathrm{D}}^{1}\left(\Omega ; \mathbb{R}^{d}\right)$. Since this equation has a unique solution, we conclude that $\bar{u}(t)=u(t)$ for almost all $t \in(0, T)$, and that the whole sequence $u_{\varepsilon_{n}}(t)$ weakly converges to $\bar{u}(t)$ for every $t \in[0, T]$. In this way $u$ extends to a function defined on $[0, T]$, such that

$$
u_{\varepsilon_{n}}(t) \rightarrow u(t) \text { in } H_{\mathrm{D}}^{1}\left(\Omega ; \mathbb{R}^{d}\right) \text { for all } t \in[0, T],
$$

solving (5.22) at all $t \in[0, T]$.

Step 2, enhanced convergences for $\left(u_{\varepsilon_{n}}\right)_{n}$ : As a by-product of this limit passage, we also extract convergences (5.39) and (5.38) below for $\left(u_{\varepsilon_{n}}\right)_{n}$, which we will then use in the passage to the limit in the semistability and in the mechanical energy inequality. Indeed, we test (5.7) by $u_{\varepsilon_{n}}$, thus obtaining

$$
\begin{aligned}
& \limsup _{n \rightarrow \infty} \int_{0}^{t} \int_{\Omega}\left(\mathbb{C}\left(z_{\varepsilon_{n}}\right) e\left(u_{\varepsilon_{n}}\right)-\theta_{\varepsilon_{n}} \mathbb{B}\right): e\left(u_{\varepsilon_{n}}\right) \mathrm{d} x \mathrm{~d} s \\
& \leq \limsup _{n \rightarrow \infty} \varepsilon_{n}^{2} \int_{0}^{t} \int_{\Omega}\left|\dot{u}_{\varepsilon_{n}}\right|^{2} \mathrm{~d} x \mathrm{~d} t-\liminf _{n \rightarrow \infty} \int_{0}^{t} \int_{\Omega} \varepsilon_{n} \mathbb{D}\left(z_{\varepsilon_{n}}, \theta_{\varepsilon_{n}}\right) e\left(\dot{u}_{\varepsilon_{n}}\right): e\left(u_{\varepsilon_{n}}\right) \mathrm{d} x \mathrm{~d} s \\
& \quad+\limsup _{n \rightarrow \infty} \varepsilon_{n}^{2} \int_{\Omega} \dot{u}_{\varepsilon_{n}}^{0} \cdot u_{\varepsilon_{n}}^{0} \mathrm{~d} x-\liminf _{n \rightarrow \infty} \varepsilon_{n}^{2} \int_{\Omega} \dot{u}_{\varepsilon_{n}}(t) \cdot u_{\varepsilon_{n}}(t) \mathrm{d} x \\
& \quad+\limsup _{n \rightarrow \infty} \int_{0}^{t}\left\langle f_{\varepsilon_{n}}, u_{\varepsilon_{n}}\right\rangle_{H_{\mathrm{D}}^{1}\left(\Omega ; \mathbb{R}^{d}\right)} \mathrm{d} s \\
& =0+0+0+0+\int_{0}^{t}\langle f, u\rangle_{H_{\mathrm{D}}^{1}\left(\Omega ; \mathbb{R}^{d}\right)} \mathrm{d} s=\int_{0}^{t} \int_{\Omega} \mathbb{C}(z) e(u): e(u) \mathrm{d} x \mathrm{~d} s
\end{aligned}
$$

where the first term in the right-hand side converges to zero thanks to (5.34), the second one by the boundedness of $\mathbb{D},(5.28 \mathrm{a})$, and (5.34), the third one by (5.35) combined with the boundedness of $\left(u_{\varepsilon_{n}}^{0}\right)_{n}$, the fourth one by (5.28a) and (5.36). The fifth term passes to the limit by (5.12) and (5.28a). The last identity follows from (5.22). Remark that the second term in the left-hand side converges to zero by (5.28a) and (5.28f), as done for (5.32).

From the above chain of inequalities we thus obtain that

$$
\limsup _{n \rightarrow \infty} \int_{0}^{t} \int_{\Omega} \mathbb{C}\left(z_{\varepsilon_{n}}\right) e\left(u_{\varepsilon_{n}}\right): e\left(u_{\varepsilon_{n}}\right) \mathrm{d} x \mathrm{~d} s \leq \int_{0}^{t} \int_{\Omega} \mathbb{C}(z) e(u): e(u) \mathrm{d} x \mathrm{~d} s .
$$

Next, we may apply Lemma 4.7 to deduce that $e\left(u_{\varepsilon_{n}}\right)$ strongly converges to $e(u)$ in $L^{2}\left((0, T) \times \Omega ; \mathbb{R}^{d \times d}\right)$, see also Lemma 4.8 . Hence, by Korn's inequality, we ultimately infer

$$
u_{\varepsilon_{n}} \rightarrow u \quad \text { in } L^{2}\left(0, T ; H_{\mathrm{D}}^{1}\left(\Omega ; \mathbb{R}^{d}\right)\right) .
$$


For later convenience, we observe that, in particular, this yields

$$
\int_{\Omega} \mathbb{C}\left(z_{\varepsilon_{n}}(t)\right) e\left(u_{\varepsilon_{n}}(t)\right): e\left(u_{\varepsilon_{n}}(t)\right) \mathrm{d} x \rightarrow \int_{\Omega} \mathbb{C}(z(t)) e(u(t)): e(u(t)) \mathrm{d} x \quad \text { for a.a. } t \in(0, T) .
$$

Step 3, passage to the limit in the semistability condition: In view of the pointwise convergences $(5.28 \mathrm{~d})-(5.28 \mathrm{e})$ for $z_{\varepsilon_{n}}$ and $u_{\varepsilon_{n}}(t) \rightarrow u(t)$ in $H_{\mathrm{D}}^{1}\left(\Omega ; \mathbb{R}^{d}\right)$ (by (5.38)) for all $t \in[0, T]$, we may apply the mutual recovery sequence construction from Theorem 4.5 in order to pass to the limit as $\varepsilon_{n} \downarrow 0$ in the semistability (5.5). Also taking into account convergence $(5.12)$ for $\left(f_{\varepsilon_{n}}\right)_{n}$, we conclude that $(u, z)$ comply with the semistability condition (2.12a) for every $t \in[0, T]$.

Step 4, passage to the limit in the mechanical energy inequality on $(0, t)$ : By lower semicontinuity it follows from convergences (5.12), (5.37), (5.28d), and (5.28c) that

$$
\liminf _{n \rightarrow \infty} \mathcal{E}_{\varepsilon_{n}}\left(t, u_{\varepsilon_{n}}(t), z_{\varepsilon_{n}}(t)\right) \geq \mathcal{E}(t, u(t), z(t)) \quad \text { for all } t \in[0, T] .
$$

Furthermore, combining (5.12) with (5.28a) we infer that

$$
\partial_{t} \varepsilon_{\varepsilon_{n}}\left(t, u_{\varepsilon_{n}}, z_{\varepsilon_{n}}\right)=-\left\langle\dot{f}_{\varepsilon_{n}}(t), u_{\varepsilon_{n}}\right\rangle_{H_{\mathrm{D}}^{1}\left(\Omega ; \mathbb{R}^{d}\right)} \rightarrow-\langle\dot{f}(t), u\rangle_{H_{\mathrm{D}}^{1}\left(\Omega ; \mathbb{R}^{d}\right)}=\partial_{t} \mathcal{E}(t, u, z) \quad \text { in } L^{2}(0, T) .
$$

We are now in a position to pass to the limit in the mechanical energy inequality (5.8). We notice that the first term on the left-hand side of (5.8) is positive. For the second one we use (5.40) and the third one converges to $\int_{\Omega}(z(0)-z(t)) \mathrm{d} x$ by (5.28e). The fourth one, given by

$$
\int_{0}^{t} \int_{\Omega}\left(\varepsilon \mathbb{D}\left(z_{\varepsilon}, \theta_{\varepsilon}\right) e\left(\dot{u}_{\varepsilon}\right)-\theta_{\varepsilon} \mathbb{B}\right): e\left(\dot{u}_{\varepsilon}\right) \mathrm{d} x \mathrm{~d} s,
$$

is bounded from below by

$$
-\int_{0}^{t} \int_{\Omega} \theta_{\varepsilon_{n}} \mathbb{B}: e\left(\dot{u}_{\varepsilon_{n}}\right) \mathrm{d} x \mathrm{~d} s
$$

We can again argue as in (5.32)

$$
\begin{aligned}
& \int_{0}^{t} \int_{\Omega} \theta_{\varepsilon_{n}} \mathbb{B}: e\left(\dot{u}_{\varepsilon_{n}}\right) \mathrm{d} x \mathrm{~d} s \\
& =\int_{0}^{t} \int_{\partial \Omega} \theta_{\varepsilon_{n}} \mathbb{B} v \cdot \dot{u}_{\varepsilon_{n}} \mathrm{~d} \mathcal{H}^{d-1}(x) \mathrm{d} s-\int_{0}^{t} \int_{\Omega} \operatorname{div}\left(\theta_{\varepsilon_{n}} \mathbb{B}\right) \cdot \dot{u}_{\varepsilon_{n}} \mathrm{~d} x \mathrm{~d} s \\
& =0-\int_{0}^{t} \int_{\Omega} \operatorname{div}\left(\theta_{\varepsilon_{n}} \mathbb{B}\right) \cdot \dot{u}_{\varepsilon_{n}} \mathrm{~d} x \mathrm{~d} s,
\end{aligned}
$$

where we have used that $\dot{u}_{\varepsilon_{n}}$ complies with homogeneous Dirichlet conditions on $\partial_{\mathrm{D}} \Omega=\partial \Omega$, and then observe that

$$
\left\|\operatorname{div}\left(\theta_{\varepsilon_{n}} \mathbb{B}\right) \cdot \dot{u}_{\varepsilon_{n}}\right\|_{L^{1}((0, T) \times \Omega)}=\left\|\varepsilon_{n}^{-1} \operatorname{div}\left(\theta_{\varepsilon_{n}} \mathbb{B}\right) \cdot \varepsilon_{n} \dot{u}_{\varepsilon_{n}}\right\|_{L^{1}((0, T) \times \Omega)} \leq C\left\|\varepsilon_{n} \dot{u}_{\varepsilon_{n}}\right\|_{L^{2}((0, T) \times \Omega)} \rightarrow 0,
$$

due to estimate (5.14g) and (5.34). Notice that here we have used the fact that $\beta \geq 2$; this is the only point where we use such requirement. As for the right-hand side, we observe that the first term converges to zero by (5.19). The second term passes to the limit by the convergence (5.20) for the initial energies, and the third one by (5.41). 
Therefore we conclude that

$$
\mathcal{E}(t, u(t), z(t))+\int_{\Omega}(z(0)-z(t)) \mathrm{d} x \leq \mathcal{E}(0, u(0), z(0))+\int_{0}^{t} \partial_{t} \mathcal{E}(s, u, z) \mathrm{d} s .
$$

Step 5, case $q>d$, enhanced convergence for $\left(z_{\varepsilon_{n}}\right)$ and energy convergence: We now prove that

$$
\lim _{n \rightarrow \infty} \int_{\Omega} G\left(z_{\varepsilon_{n}}(t), \nabla z_{\varepsilon_{n}}(t)\right) \mathrm{d} x=\int_{\Omega} G(z(t), \nabla z(t)) \mathrm{d} x \quad \text { for a.a. } t \in(0, T),
$$

which, combined with (5.12), (5.39) and (5.38) will yield the pointwise convergence of the energies

$$
\lim _{n \rightarrow \infty} \varepsilon_{\varepsilon_{n}}\left(t, u_{\varepsilon_{n}}(t), z_{\varepsilon_{n}}(t)\right)=\mathcal{E}(t, u(t), z(t)) \quad \text { for a.a. } t \in(0, T) .
$$

We obtain (5.44) testing semistability (5.5) by a suitable recovery sequence $\left(\tilde{z}_{\varepsilon_{n}}\right)_{n}$ for $\tilde{z}=z(t)$; in the following lines, to avoid overburdening notation we will drop $t$ when writing $z_{\varepsilon_{n}}(t), z(t), u_{\varepsilon_{n}}(t)$, and $u(t)$. Following [44, Lemma 3.9], where the recovery sequence right below has been introduced to deduce energy convergence, we set

$$
\tilde{z}_{\varepsilon_{n}}:=\max \left\{0, z-\left\|z_{\varepsilon_{n}}-z\right\|_{L^{\infty}(\Omega)}\right\} .
$$

Now, for $q>d$ the convergence $z_{\varepsilon_{n}} \rightarrow z$ in $W^{1, q}(\Omega)$, see (5.28d), implies $z_{\varepsilon_{n}} \rightarrow z$ in $L^{\infty}(\Omega)$. Thus, it can be checked that

$$
\tilde{z}_{\varepsilon_{n}} \rightarrow z \text { strongly in } W^{1, q}(\Omega) .
$$

Since $\tilde{z}_{\varepsilon_{n}} \leq z_{\varepsilon_{n}}$, we can choose it as a test function in (5.5). The term $-\left\langle f_{\varepsilon_{n}}(t), u_{\varepsilon_{n}}\right\rangle_{H_{\mathrm{D}}^{1}\left(\Omega ; \mathbb{R}^{d}\right)}$ on both sides of the inequality cancels out and we deduce

$$
\begin{aligned}
& \limsup _{n \rightarrow \infty}\left(\int_{\Omega}\left(\frac{1}{2} \mathbb{C}\left(z_{\varepsilon_{n}}\right) e\left(u_{\varepsilon_{n}}\right): e\left(u_{\varepsilon_{n}}\right)+G\left(z_{\varepsilon_{n}}, \nabla z_{\varepsilon_{n}}\right)\right) \mathrm{d} x\right) \\
& =\limsup _{n \rightarrow \infty}\left(\int_{\Omega} \frac{1}{2} \mathbb{C}\left(\tilde{z}_{n}\right) e\left(u_{\varepsilon_{n}}\right): e\left(u_{\varepsilon_{n}}\right) \mathrm{d} x+\int_{\Omega} G\left(\tilde{z}_{\varepsilon_{n}}, \nabla \tilde{z}_{\varepsilon_{n}}\right) \mathrm{d} x\right) \leq I_{1}+I_{2},
\end{aligned}
$$

where

$$
I_{1}:=\lim _{n \rightarrow \infty} \int_{\Omega} \frac{1}{2} \mathbb{C}\left(\tilde{z}_{n}\right) e\left(u_{\varepsilon_{n}}\right): e\left(u_{\varepsilon_{n}}\right) \mathrm{d} x \leq \int_{\Omega} \frac{1}{2} \mathbb{C}(z) e(u): e(u) \mathrm{d} x,
$$

combining (5.46) with (5.38) via the Lebesgue Theorem. It follows from (5.46), condition (2.5d) on the growth of $G$ from above, and again the Lebesgue Theorem that

$$
I_{2}:=\lim _{n \rightarrow \infty} \int_{\Omega} G\left(\tilde{z}_{\varepsilon_{n}}, \nabla \tilde{z}_{\varepsilon_{n}}\right) \mathrm{d} x=\int_{\Omega} G(z, \nabla z) \mathrm{d} x .
$$

Taking into account the previously proven (5.39), from (5.47)-(5.48) we ultimately infer

$$
\limsup _{n \rightarrow \infty} \int_{\Omega} G\left(z_{\varepsilon_{n}}, \nabla z_{\varepsilon_{n}}\right) \mathrm{d} x \leq \int_{\Omega} G(z, \nabla z) \mathrm{d} x
$$

whence (5.44).

Step 6, case $q>d$, passage to the limit in the mechanical energy inequality on $(s, t)$ : We now pass to the limit in (5.8) written on an interval $[s, t] \subset[0, T]$, for every $t \in[0, T]$ and almost all $s \in(0, t)$. Clearly, it is sufficient to discuss the limit passage on the right-hand side of (5.8), evaluated at $s$. The first summand tends to zero for almost all $s$, thanks to (5.34), which in particular ensures $\varepsilon_{n} \dot{u}_{\varepsilon_{n}}(s) \rightarrow 0$ in $L^{2}\left(\Omega ; \mathbb{R}^{d}\right)$ for almost all $s \in(0, T)$. The second

\section{黛 Springer}


term passes to the limit by (5.45), while the third and the fourth ones can be dealt with by (5.42)-(5.43) and (5.41), respectively.

Step 7, limit passage in the rescaled heat equation and temporal evolution of $\Theta$ : We consider the heat equation (5.9) rescaled by the factor $1 / \varepsilon$ and tested by $\eta \in H^{1}(0, T)$, constant in space, which results in

$$
\begin{aligned}
\eta(t) & \int_{\Omega} \theta_{\varepsilon}(t) \mathrm{d} x-\int_{0}^{t} \dot{\eta} \int_{\Omega} \theta_{\varepsilon} \mathrm{d} x \mathrm{~d} s \\
= & \eta(0) \int_{\Omega} \theta_{\varepsilon}^{0} \mathrm{~d} x+\int_{0}^{t} \eta \int_{\Omega}\left(\varepsilon \mathbb{D}\left(z_{\varepsilon}, \theta_{\varepsilon}\right) e\left(\dot{u}_{\varepsilon}\right)-\theta_{\varepsilon} \mathbb{B}\right): e\left(\dot{u}_{\varepsilon}\right) \mathrm{d} x \mathrm{~d} s \\
& +\int_{0}^{t} \eta \int_{\Omega}\left|\dot{z}_{\varepsilon}\right| \mathrm{d} x \mathrm{~d} s+\frac{1}{\varepsilon} \int_{0}^{t} \eta \int_{\partial \Omega} h_{\varepsilon} \mathrm{d} \mathcal{H}^{d-1}(x) \mathrm{d} s+\frac{1}{\varepsilon} \int_{0}^{t} \eta \int_{\Omega} H_{\varepsilon} \mathrm{d} x \mathrm{~d} s .
\end{aligned}
$$

From the mechanical energy balance (5.8) we deduce by a comparison argument that

$$
\begin{gathered}
\varepsilon \int_{0}^{T} \int_{\Omega} \mathbb{D}\left(z_{\varepsilon}, \theta_{\varepsilon}\right) e\left(\dot{u}_{\varepsilon}\right): e\left(\dot{u}_{\varepsilon}\right) \mathrm{d} x \mathrm{~d} s \leq C, \text { hence also } \\
\varepsilon \int_{0}^{T} \eta \int_{\Omega} \mathbb{D}\left(z_{\varepsilon}, \theta_{\varepsilon}\right) e\left(\dot{u}_{\varepsilon}\right): e\left(\dot{u}_{\varepsilon}\right) \mathrm{d} x \mathrm{~d} s \leq C\|\eta\|_{\infty}
\end{gathered}
$$

for every $\eta \in H^{1}(0, T)$, taking into account (5.12), (5.13) as well as (5.18). This allows us to conclude that there exists a Radon measure $\mu$ such that (5.27) holds. A comparison argument in (5.49) leads to

$$
\left|\varepsilon \int_{0}^{t} \eta \int_{\Omega} \theta_{\varepsilon} \mathbb{B}: e\left(\dot{u}_{\varepsilon}\right) \mathrm{d} x \mathrm{~d} s\right| \leq C\|\eta\|_{\infty},
$$

also in view of the bounds $(5.11),(5.14 \mathrm{i})$ and $(5.14 \mathrm{c})$. Since $\eta$ is constant in space, integration by parts and an argument along the lines of Step 4 yield that indeed $\int_{0}^{t} \int_{\Omega} \eta \theta_{\varepsilon} \mathbb{B}$ : $e\left(\dot{u}_{\varepsilon}\right) \mathrm{d} x \mathrm{~d} s \rightarrow 0$. Moreover, the third convergence in (5.18) implies that $\theta_{\varepsilon}(t) \rightarrow \Theta(t)$ in $L^{2}(\Omega)$ for a.e. $t \in(0, T)$. Using (5.25), we finally pass to the limit in (5.49) and find that $\Theta$ satisfies (5.26).

Acknowledgements This work has been supported by the Italian Ministry of Education, University, and Research through the PRIN 2010-11 grant for the project Calculus of Variations, by the European Research Council through the two Advanced Grants Quasistatic and Dynamic Evolution Problems in Plasticity and Fracture (290888) and Analysis of Multiscale Systems Driven by Functionals (267802), and by GNAMPA (Gruppo Nazionale per l'Analisi Matematica, la Probabilità e le loro Applicazioni) of INdAM (Istituto Nazionale di Alta Matematica) through the project Modelli variazionali per la propagazione di fratture, la delaminazione $e$ il danneggiamento. G.L. acknowledges also the support of the University of Würzburg, of the DFG grant SCHL 1706/2-1, of SISSA, of the University of Vienna, and of the FWF project P27052. This paper was submitted on October 24, 2014; the first referee report was received by the authors on December 6, 2017.

\section{References}

1. Agostiniani, V.: Second order approximations of quasistatic evolution problems in finite dimension. Discrete Contin. Dyn. Syst. 32(4), 1125-1167 (2012)

2. Ambrosio, L., Tortorelli, V.M.: Approximation of functionals depending on jumps by elliptic functionals via $\Gamma$-convergence. Commun. Pure Appl. Math. 43(8), 999-1036 (1990)

3. Bonetti, E., Bonfanti, G.: Well-posedness results for a model of damage in thermoviscoelastic materials. Ann. Inst. H. Poincaré Anal. Non Linéaire 25(6), 1187-1208 (2008)

4. Bourdin, B., Francfort, G.A., Marigo, J.-J.: The variational approach to fracture. J. Elast. 91(1-3), 5-148 (2008) 
5. Babadjian, J.-F., Millot, V.: Unilateral gradient flow of the Ambrosio-Tortorelli functional by minimizing movements. Ann. Inst. H. Poincaré Anal. Non Linéaire 31(4), 779-822 (2014)

6. Bouchitté, G., Mielke, A., Roubíček, T.: A complete-damage problem at small strain. Zeit. Angew. Math. Phys. 60, 205-236 (2009)

7. Bartels, S., Roubíček, T.: Thermo-visco-elasticity with rate-independent plasticity in isotropic materials undergoing thermal expansion. ESAIM Math. Model. Numer. Anal. 45(3), 477-504 (2011)

8. Bonetti, E., Schimperna, G.: Local existence for Frémond's model of damage in elastic materials. Contin. Mech. Thermodyn. 16(4), 319-335 (2004)

9. Bonetti, E., Schimperna, G., Segatti, A.: On a doubly nonlinear model for the evolution of damaging in viscoelastic materials. J. Differ. Equ. 218(1), 91-116 (2005)

10. Burenkov, V.I.: Sobolev Spaces on Domains, vol. 137. B. G. Teubner, Leipzig (1998)

11. Callister, W.D., Rethwisch, D.G.: Fundamentals of Materials Science and Engineering: An Integrated Approach, 4th edn. Wiley, Hoboken (2012)

12. Dal Maso, G., DeSimone, A., Solombrino, F.: Quasistatic evolution for Cam-Clay plasticity: a weak formulation via viscoplastic regularization and time rescaling. Calc. Var. Partial Differ. Equ. 40(1-2), 125-181 (2011)

13. Dal Maso, G., Francfort, G.A., Toader, R.: Quasistatic crack growth in nonlinear elasticity. Arch. Ration. Mech. Anal. 176(2), 165-225 (2005)

14. Dal Maso, G., Lazzaroni, G.: Quasistatic crack growth in finite elasticity with non-interpenetration. Ann. Inst. H. Poincaré Anal. Non Linéaire 27(1), 257-290 (2010)

15. Dal Maso, G., Scala, R.: Quasistatic evolution in perfect plasticity as limit of dynamic processes. J. Dyn. Differ. Equ. 26(4), 915-954 (2014)

16. Eiermann, K.: Modellmäßige deutung der wärmeleitfähigkeit von hochpolymeren, teil2: Verstreckte amorphe hochpolymere. Kolloid-Zeitschrift und Zeitschrift für Polymere 199(2), 125-128 (1964)

17. Efendiev, M.A., Mielke, A.: On the rate-independent limit of systems with dry friction and small viscosity. J. Convex Anal. 13(1), 151-167 (2006)

18. Francfort, G.A., Garroni, A.: A variational view of partial brittle damage evolution. Arch. Ration. Mech. Anal. 182(1), 125-152 (2006)

19. Fiaschi, A., Knees, D., Stefanelli, U.: Young-measure quasi-static damage evolution. Arch. Ration. Mech. Anal. 203(2), 415-453 (2012)

20. Francfort, G.A., Larsen, C.J.: Existence and convergence for quasi-static evolution in brittle fracture. Commun. Pure Appl. Math. 56(10), 1465-1500 (2003)

21. Fonseca, I., Leoni, G.: Modern Methods in the Calculus of Variations: $L^{p}$ Spaces. Springer, New York (2007)

22. Frémond, M., Nedjar, B.: Damage, gradient of damage and principle of virtual power. Int. J. Solids Struct. 33, 1083-1103 (1996)

23. Feireisl, E., Petzeltová, H., Rocca, E.: Existence of solutions to a phase transition model with microscopic movements. Math. Methods Appl. Sci. 32(11), 1345-1369 (2009)

24. Frémond, M.: Non-Smooth Thermomechanics. Springer-Verlag, Berlin, Heidelberg (2002)

25. Giacomini, A.: Ambrosio-Tortorelli approximation of quasi-static evolution of brittle fracture. Calc. Var. Part. Differ. Equ. 22, 129-172 (2005)

26. Garroni, A., Larsen, C.J.: Threshold-based quasi-static brittle damage evolution. Arch. Ration. Mech. Anal. 194(2), 585-609 (2009)

27. Hahn, H.: Über Annäherung an Lebesgue'sche Integrale durch Riemann'sche Summen. Sitzungsber. Math. Phys. Kl. K. Akad. Wiss. Wien 123, 713-743 (1914)

28. Heinemann, C., Kraus, C.: Complete damage in linear elastic materials: modeling, weak formulation and existence results. Calc. Var. Part. Differ. Equ. 54(1), 217-250 (2015)

29. Halphen, B., Nguyen, Q.S.: Sur les matériaux standards généralisés. J. Mécanique 14, 39-63 (1975)

30. Iurlano, F.: Fracture and plastic models as $\Gamma$-limits of damage models under different regimes. Adv. Calc. Var. 6(2), 165-189 (2013)

31. Klein, R.: Laser Welding of Plastics. Wiley-VCH, Hoboken (2012)

32. Kočvara, M., Mielke, A., Roubíček, T.: A rate-independent approach to the delamination problem. Math. Mech. Solids 11, 423-447 (2006)

33. Knees, D., Mielke, A., Zanini, C.: On the inviscid limit of a model for crack propagation. Math. Models Methods Appl. Sci. 18(9), 1529-1569 (2008)

34. Knees, D., Rossi, R., Zanini, C.: A vanishing viscosity approach to a rate-independent damage model. Math. Models Methods Appl. Sci. 23(4), 565-616 (2013)

35. Lemaitre, J., Desmorat, R.: Engineering Damage Mechanics: Ductile, Creep, Fatigue and Brittle Failures. Springer, New York (2005) 
36. Lions, J.-L., Magenes, E.: Non-homogeneous Boundary Value Problems and Applications, vol. I. Springer-Verlag, Berlin, Heidelberg (1972)

37. Larsen, C.J., Ortner, C., Süli, E.: Existence of solutions to a regularized model of dynamic fracture. Math. Models Methods Appl. Sci. 20(7), 1021-1048 (2010)

38. Lazzaroni, G., Rossi, R., Thomas, M., Toader, R.: Some remarks on a model for rate-independent damage in thermo-visco-elastodynamics. J. Phys. Conf. Ser 727, 012009, 20 (2016)

39. Lazzaroni, G., Toader, R.: A model for crack propagation based on viscous approximation. Math. Models Methods Appl. Sci. 21(10), 2019-2047 (2011)

40. Mielke, A.: Evolution in rate-independent systems (Ch.6). In: Dafermos, C.M., Feireisl, E. (eds.) Handbook of Differential Equations, Evolutionary Equations, vol. 2, pp. 461-559. Elsevier B.V, Amsterdam (2005)

41. Mielke, A.: Differential, energetic and metric formulations for rate-independent processes (Ch. 3). In: Ambrosio, L., Savaré, G. (eds.) Nonlinear PDEs and Applications.C.I.M.E. Summer School, Cetraro, Italy 2008, pp. 87-170. Springer, Heidelberg (2011)

42. Martins, J.A.C., Monteiro Marques, M.D.P., Petrov, A.: On the stability of quasi-static paths for finite dimensional elastic-plastic systems with hardening. ZAMM Z. Angew. Math. Mech. 87(4), 303-313 (2007)

43. Mielke, A., Petrov, A., Martins, J.A.C.: Convergence of solutions of kinetic variational inequalities in the rate-independent quasi-static limit. J. Math. Anal. Appl. 348(2), 1012-1020 (2008)

44. Mielke, A., Roubíček, T.: Rate-independent damage processes in nonlinear elasticity. Math. Models Methods Appl. Sci. 16(2), 177-209 (2006)

45. Mielke, A., Roubíček, T.: Rate-Independent Systems. Theory and Application, volume 193 of Applied Mathematical Sciences. Springer, New York (2015)

46. Martins, J.A.C., Rebrova, N.V., Sobolev, V.A.: On the (in)stability of quasi-static paths of smooth systems: definitions and sufficient conditions. Math. Methods Appl. Sci. 29(6), 741-750 (2006)

47. Mielke, A., Roubíček, T., Stefanelli, U.: $\Gamma$-limits and relaxations for rate-independent evolutionary problems. Calc. Var. Part. Differ. Equ. 31, 387-416 (2008)

48. Mielke, A., Rossi, R., Savaré, G.: Modeling solutions with jumps for rate-independent systems on metric spaces. Discrete Contin. Dyn. Syst. 25(2), 585-615 (2009)

49. Mielke, A., Rossi, R., Savaré, G.: BV solutions and viscosity approximations of rate-independent systems. ESAIM Control Optim. Calc. Var. 18(1), 36-80 (2012)

50. Martins, J.A.C., Simões, F.M.F., Gastaldi, F., Monteiro Marques, M.D.P.: Dissipative graph solutions for a 2 degree-of-freedom quasistatic frictional contact problem. Int. J. Eng. Sci. 33(13), 1959-1986 (1995)

51. Mielke, A., Theil, F.: On rate-independent hysteresis models. NoDEA Nonlinear Differ. Equ. Appl. 11(2), 151-189 (2004)

52. Miehe, C., Welschinger, F., Hofacker, M.: Thermodynamically consistent phase-field models of fracture: variational principles and multi-field FE implementations. Int. J. Numer. Methods Eng. 83(10), 1273-1311 (2010)

53. Nardini, L.: A note on the convergence of singularly perturbed second order potential-type equations. J. Dyn. Differ. Equ. 29(2), 783-797 (2017)

54. Nečas, J., Štípl, M.: A paradox in the theory of linear elasticity. Appl. Mat. 21, 431-433 (1976)

55. Royden, H.L., Fitzpatrick, P.M.: Real Analysis, 4th edn. Prentice Hall, Boston (2010)

56. Roubíček, T.: Rate-independent processes in viscous solids at small strains. Math. Methods Appl. Sci. 32(7), 825-862 (2009)

57. Roubíček, T.: Thermodynamics of rate independent processes in viscous solids at small strains. SIAM J. Math. Anal. 40, 256-297 (2010)

58. Roubíček, T.: Adhesive contact of visco-elastic bodies and defect measures arising by vanishing viscosity. SIAM J. Math. Anal. 45(1), 101-126 (2013)

59. Roubíček, T.: Nonlinearly coupled thermo-visco-elasticity. NoDEA Nonlinear Differ. Equ. Appl. 20(3), $1243-1275$ (2013)

60. Roubíček, T.: Nonlinear Partial Differential Equations with Applications, volume 153 of International Series of Numerical Mathematics, 2nd edn. Birkhäuser/Springer Basel AG, Basel (2013)

61. Rossi, R., Roubíček, T.: Thermodynamics and analysis of rate-independent adhesive contact at small strains. Nonlinear Anal. 74(10), 3159-3190 (2011)

62. Rocca, E., Rossi, R.: A degenerating PDE system for phase transitions and damage. Math. Models Methods Appl. Sci. 24(7), 1265-1341 (2014)

63. Rocca, E., Rossi, R.: "Entropic" solutions to a thermodynamically consistent PDE system for phase transitions and damage. SIAM J. Math. Anal. 47(4), 2519-2586 (2015)

64. Roubíček, T., Tomassetti, G.: Thermomechanics of damageable materials under diffusion: modelling and analysis. Z. Angew. Math. Phys. 66(6), 3535-3572 (2015) 
65. Roubíček, T., Thomas, M., Panagiotopoulos, C.: Stress-driven local-solution approach to quasistatic brittle delamination. Nonlinear Anal. Real World Appl. 22, 645-663 (2015)

66. Scala, R.: Limit of viscous dynamic processes in delamination as the viscosity and inertia vanish. ESAIM Control Optim. Calc. Var. 23(2), 593-625 (2017)

67. Thomas, M.: Quasistatic damage evolution with spatial BV-regularization. Discrete Contin. Dyn. Syst. Ser. S 6, 235-255 (2013)

68. Thomas, M., Mielke, A.: Damage of nonlinearly elastic materials at small strain: existence and regularity results. Zeit. angew. Math. Mech. 90(2), 88-112 (2010)

69. Wedler, G.: Lehrbuch der physikalischen Chemie, 4th edn. Wiley-VCH, Hoboken (1997)

70. Zel'dovič, J.B., Rajzer, J.P.: Physics of Shock Waves and High-Temperature Hydrodynamic Phenomena. Dover, Illinois (2002) 